\title{
NEWTON POLYHEDRA AND WEIGHTED OSCILLATORY INTEGRALS WITH SMOOTH PHASES
}

\author{
JOE KAMIMOTO AND TOSHIHIRO NOSE
}

ABstract. In his seminal paper, A. N. Varchenko precisely investigates the leading term of the asymptotic expansion of an oscillatory integral with real analytic phase. He expresses the order of this term by means of the geometry of the Newton polyhedron of the phase. The purpose of this paper is to generalize and improve his result. We are especially interested in the cases that the phase is smooth and that the amplitude has a zero at a critical point of the phase. In order to exactly treat the latter case, a weight function is introduced in the amplitude. Our results show that the optimal rates of decay for weighted oscillatory integrals whose phases and weights are contained in a certain class of smooth functions, including the real analytic class, can be expressed by the Newton distance and multiplicity defined in terms of geometrical relationship of the Newton polyhedra of the phase and the weight. We also compute explicit formulae of the coefficient of the leading term of the asymptotic expansion in the weighted case. Our method is based on the resolution of singularities constructed by using the theory of toric varieties, which naturally extends the resolution of Varchenko. The properties of poles of local zeta functions, which are closely related to the behavior of oscillatory integrals, are also studied under the associated situation. The investigation of this paper improves on the earlier joint work with K. Cho.

\section{Contents}

1. Introduction

2. Newton polyhedra and the class $\hat{\mathcal{E}}(U)$

3. Earlier studies

4. Main results

5. Toric varieties

6. Construction of fans from polyhedra

7. Resolution of singularities in the class $\hat{\mathcal{E}}(U)$

8. Poles of local zeta type functions

9. Computation on poles of the elementary integrals 5326

10. The case of monomial type weight $\quad 5329$

11. The case of $\hat{\mathcal{E}}$-weight

12. The case of convenient $f \quad 5348$

13. Certain symmetry properties $\quad 5352$

14. Proofs of the theorems in Section 4

Received by the editors August 26, 2013 and, in revised form, June 9, 2014.

2010 Mathematics Subject Classification. Primary 58K55; Secondary 42B20, 14 M25.

Key words and phrases. Weighted oscillatory integrals, oscillation index and its multiplicity, resolution of singularities, Newton polyhedron, Newton distance, Newton multiplicity, principal faces.

The first author was supported by Grant-in-Aid for Scientific Research (C) (No. 22540199), Japan Society for the Promotion of Science. 
15. Examples

\begin{tabular}{ll} 
Acknowledgements & 5359 \\
\hline
\end{tabular}

References $\quad 5359$

\section{INTRODUCTION}

In this paper, we investigate the asymptotic behavior of scalar oscillatory integrals of the weighted form

$$
I(t ; \varphi)=\int_{\mathbb{R}^{n}} e^{i t f(x)} g(x) \varphi(x) d x
$$

for large values of the real parameter $t$, where $f, g, \varphi$ are real-valued smooth (infinitely differentiable) functions defined on an open neighborhood $U$ of the origin in $\mathbb{R}^{n}$ and

- $f$ is called the phase and satisfies that $f(0)=|\nabla f(0)|=0$;

- $g$ is called the weight ( $g \varphi$ is called the amplitude);

- the support of $\varphi$ is contained in $U$.

By using Hironaka's famous resolution of singularities [13, it is known (see [19, 22. and Section 8.2 in this paper) that if $f$ is real analytic and the support of $\varphi$ is contained in a sufficiently small open neighborhood of the origin, then the integral $I(t ; \varphi)$ has an asymptotic expansion of the form

$$
I(t ; \varphi) \sim \sum_{\alpha} \sum_{k=1}^{n} C_{\alpha k}(\varphi) t^{\alpha}(\log t)^{k-1} \quad \text { as } t \rightarrow+\infty,
$$

where $\alpha$ runs through a finite number of arithmetic progressions, not depending on the amplitude, which consist of negative rational numbers. In special cases of the smooth phase, $I(t ; \varphi)$ also admits an asymptotic expansion of the same form as in (1.2) (see [27], 20] and Theorem 3.7 in this paper). In order to see the decay property of $I(t ; \varphi)$, we are interested in the leading term of (1.2) and define the following index.

Definition 1.1. Let $f, g$ be smooth functions for which the oscillatory integral (1.1) admits the asymptotic expansion of the form (1.2). The set $S(f, g)$ consists of pairs $(\alpha, k)$ such that for each neighborhood of the origin in $\mathbb{R}^{n}$, there exists a smooth function $\varphi$ with support contained in this neighborhood for which $C_{\alpha k}(\varphi) \neq 0$ in (1.2). The maximum element of the set $S(f, g)$, under the lexicographic ordering, is denoted by $(\beta(f, g), \eta(f, g))$; i.e., $\beta(f, g)$ is the maximum of values $\alpha$ for which we can find $k$ so that $(\alpha, k)$ belongs to $S(f, g)$, and $\eta(f, g)$ is the maximum of integers $k$ satisfying that $(\beta(f, g), k)$ belongs to $S(f, g)$. We call $\beta(f, g)$ the oscillation index of $(f, g)$ and $\eta(f, g)$ the multiplicity of its index. (In the unweighted case, i.e., $g \equiv 1$, the multiplicity $\eta(f, 1)$ is one less than the corresponding multiplicity in [1, p. 183].)

The aim of this paper is to determine or precisely estimate the oscillation index and its multiplicity by means of appropriate information of the phase and the weight. In the unweighted case, many strong results have been obtained. In particular, in a seminal work of Varchenko [30] (see also [1]), the oscillation index and its multiplicity are investigated in detail in the case when the phase is real analytic and satisfies a certain nondegeneracy condition (see Theorem 3.1 in Section 33). 
In his analysis, the theory of toric varieties based on the geometry of the Newton polyhedron of the phase plays an important role. Recently, it was shown in [20] that the above result of Varchenko can be generalized to the case that the phase belongs to a wider class of smooth functions, denoted by $\hat{\mathcal{E}}(U)$, including the real analytic class (see Theorem 3.8 in Section 3). On the other hand, another approach, which is inspired by the work of Phong and Stein on oscillatory integral operators in the seminal paper 25], has been developed and succeeds in giving many strong results ([10], 11], [12, [14, 16, 6], etc.). In particular, the two-dimensional case has been deeply understood. In these papers, the importance of resolution of singularities constructed from the Newton polyhedron is also strongly recognized.

Before explaining earlier works in the weighted case, we comment on the significance for the investigation in this case. Since the weighted case may be considered as a special case of the unweighted case, unweighted results concerned with the upper bound estimates for the oscillation index are also available in the weighted case. But, these are "uniformly" satisfied with respect to the amplitude, so more precise results may be obtained in the case of a specific amplitude. Therefore, for a given weight, one must try to get more accurate results by means of not only the information of the phase but also that of the weight.

Until now, there have not been many studies about the weighted case, but some precise results have been obtained in [31, [1], [26], [5], 24]. In these studies, the Newton polyhedra of both the phase and the weight play important roles. In 31, [1, 5], 24] an attempt was made to generalize the results of Varchenko in [30] as directly as possible in the weighted case under the nondegeneracy condition on the phase. Pramanik and Yang [26] consider the two-dimensional case with the weight of the form $g(x)=|h(x)|^{\epsilon}$, where $h$ is real analytic and $\epsilon$ is positive. (This $g$ may not be smooth.) Their approach is based not only on the method of Varchenko but also on the above-mentioned work of Phong and Stein in [25]. As a result, they succeed in removing the nondegeneracy hypothesis on the phase. Note that every study mentioned above requires the assumption of the real analyticity of the phase.

The purpose of this paper is to improve results in our previous paper [5, which generalizes the above-mentioned results of Varchenko in 30 to the weighted case. First, we develop the investigation in [5] into the case of smooth phases. When the case of smooth functions is treated, it must be noticed that the geometrical information of the Newton polyhedra does not always give sufficient analytical information. To be more specific, though flat functions do not appear in the information of the Newton polyhedron, they may affect the behavior of the oscillatory integrals. In particular, we must be careful in dealing with the case when the complement of their Newton polyhedra in $\mathbb{R}_{+}^{n}$ is noncompact. Actually, we recall that since smooth weights were considered in [5], the results needed many intricate hypotheses to avoid various troubles induced by noncompact faces of the Newton polyhedra (see Theorems 3.2 and 3.3 in this paper). Additionally considering the case of smooth phases, one must overcome more complicated problems. In this paper, we use the above-mentioned function class $\hat{\mathcal{E}}(U)$ in the investigation of the weighted case and succeed in generalizing the above results in [5]. Indeed, we show that the real analyticity can be replaced by the $\hat{\mathcal{E}}$-property in the assumption on the phase; many conditions can be written clearly in terms of the $\hat{\mathcal{E}}$-property in the assumption on the weight in the previous paper [5]. But, there is still room for improvement for the refined condition of the weight, and the subtlety of conditions of the weight will be explained in terms of some examples (see Section 15). 
Here, we briefly explain properties of the class $\hat{\mathcal{E}}(U)$, which were investigated in detail in 20. In many earlier investigations of oscillatory integrals, the function $\gamma$-part, corresponding to each face $\gamma$ of the Newton polyhedron, plays an important role. By means of summation, the $\gamma$-part is simply defined as a function for every face $\gamma$ in the real analytic case. From the viewpoint of this definition, the $\gamma$-part is considered as a formal power series when $\gamma$ is noncompact in the smooth case. This $\gamma$-part may not become a function, so it is not useful for our analysis. From convex geometrical points of view (cf. 32]), we give another definition of the $\gamma$-part, which always becomes a function defined near the origin (see Section 2.4). This definition is a natural generalization of that in the real analytic case. We remark that not all smooth functions admit the $\gamma$-part for every face $\gamma$ of their Newton polyhedra in our sense. The class $\hat{\mathcal{E}}(U)$ is defined to be the set of smooth functions admitting the $\gamma$-part for every face $\gamma$ of its Newton polyhedron (see Section 2.5). Many kinds of $C^{\infty}$ functions are contained in this class. In particular, it is shown in 20] that the class $\hat{\mathcal{E}}(U)$ contains the Denjoy-Carleman quasianalytic classes, which are interesting classes of smooth functions and have been studied from various points of view (cf. 4], 29]).

Let us explain another improvement on the previous studies in [5]. As mentioned above, the importance of resolution of singularities has been strongly recognized in earlier successive investigations of the behavior of oscillatory integrals. Let us review our analysis from this point of view. The resolution in the work of Varchenko 30] is based on the theory of toric varieties. His method gives quantitative resolution by means of the geometry of the Newton polyhedron of the phase. In 20, we have directly generalized this resolution to the class $\hat{\mathcal{E}}(U)$ of smooth functions. Furthermore, in order to consider the weighted case, some kind of $s i$ multaneous resolution of singularities with respect to two functions, i.e., the phase and the weight, must be constructed (see Section 8.2). From the viewpoint of the theory of toric varieties, simultaneous resolution of singularities reflects finer simplicial subdivision of a fan constructed from the Newton polyhedra of the above two functions. Therefore, it is essentially important to investigate an accurate relationship between cones of this subdivided fan and faces of the Newton polyhedra of the two functions. This situation has been investigated in [5], but deeper understanding of this relationship in this paper (see Section 11.2) gives many stronger results about the behavior of oscillatory integrals. In particular, we succeed in giving explicit formulae of the coefficient of the leading term of the asymptotic expansion under some appropriate conditions, which reveals that the behavior of oscillatory integrals is decided by some important faces, which are called principal faces, of the Newton polyhedra of the phase and the weight. By the way, Pramanik and Yang [26] also use simultaneous resolution of singularities in their analysis. Indeed, they extend the method of Phong and Stein [25] by considering the expressions of both the phase and the weight in terms of the Puiseux series of their roots.

It is known (see, for instance, 18, 1], and Section 14.1 in this paper) that the asymptotic analysis of oscillatory integral (1.1) can be reduced to an investigation of the poles of the functions $Z_{+}(s ; \varphi)$ and $Z_{-}(s ; \varphi)$ (see (8.1) below), which are similar to the (weighted) local zeta function

$$
Z(s ; \varphi)=\int_{\mathbb{R}^{n}}|f(x)|^{s} g(x) \varphi(x) d x,
$$


where $f, g, \varphi$ are the same as in (1.1). The substantial analysis in this paper is to investigate properties of poles of the local zeta function $Z(s ; \varphi)$ and the functions $Z_{ \pm}(s ; \varphi)$ by means of the Newton polyhedra of the functions $f$ and $g$. We will give analogous new results about properties of poles of these functions.

This paper is organized as follows. In Section 2, we explain many important words and their elementary properties, which are often used in this paper. First, fundamental notions in convex geometry are recalled. Second, after recalling the concept of Newton polyhedra of smooth functions, we give definitions of key words: Newton distance, Newton multiplicity and principal faces. Third, we define the classes $\hat{\mathcal{E}}[P](U)$ and $\hat{\mathcal{E}}(U)$ of smooth functions by means of modified $\gamma$-parts and summarize important properties of these classes. In Section 3, in order to clarify original parts of this paper, we roughly explain earlier corresponding results in [30, [31, 1], 26], 5], 20]. In Section 4, our main results relating to the behavior of oscillatory integrals are stated. Note that the result concerning explicit formulae of the coefficient of the leading term of the asymptotic expansion (1.2) will be given in Section 14. The goal of the next three sections is to construct resolution of singularities with respect to several functions by using the theory of toric varieties. First, we briefly recall how to construct a toric variety from a given fan in Section 5 . In Section 6, the construction of fans from a given polyhedron is explained. Here, we give a few lemmas which reveal important relationships of faces of polyhedron and cones of the fan. Finally, simultaneous resolution of singularities with respect to several functions is constructed in Section 7 . In the next six sections, the properties of poles of local zeta function $Z(s ; \varphi)$ and similar functions $Z_{+}(s ; \varphi)$ and $Z_{-}(s ; \varphi)$ are investigated under the assumption that $f$ belongs to the class $\hat{\mathcal{E}}(U)$ and satisfies some nondegeneracy condition. In Section 8, we roughly explain how to understand required properties of poles by means of resolution of singularities. In Section 9, the model case " $f$ and $g$ in (1.3) are monomials" is exactly investigated. In this case, it is easy to compute the coefficient of the Laurent expansion of the leading pole. In Section 10, we treat the weight $g$ of a special form: a monomial multiplied by a smooth function. By using results in this case, we can generalize the results about unweighted local zeta type functions due to Varchenko [30] and Kamimoto and Nose [20] in the case when $f$ admits an asymptotic expansion at the origin of the form of fractional power series. In Section 11, a more general case when $g$ belongs to the class $\hat{\mathcal{E}}(U)$ is investigated, and the strongest result concerning the poles of local zeta type functions is given. By the way, the case when $f$ is convenient (see Section 2.2) is easy to treat. This situation is explained in Section 12. In Section 13, we consider the question: what will happen in the positions of the leading poles of $Z(s ; \varphi)$ if $f$ and $g$ are exchanged? In Section 14, after an exact relationship between oscillatory integrals and local zeta type functions in terms of the Mellin transform is recalled, the results in the preceding six sections are translated into those in the case of oscillatory integrals. As a result, proofs of the theorems in Section 4 are given. In the last section, we give some examples which show the subtlety of the condition of the weight in the assumption of the main theorems.

Some of the results in this paper have been announced in 21].

Notation and symbols.

- We denote by $\mathbb{Z}_{+}, \mathbb{Q}_{+}, \mathbb{R}_{+}$the subsets consisting of all nonnegative numbers in $\mathbb{Z}, \mathbb{Q}, \mathbb{R}$, respectively. We write $\mathbb{R}_{>0}:=\{x \in \mathbb{R}: x>0\}$. For $s \in \mathbb{C}$, $\operatorname{Re}(s)$ expresses the real part of $s$. 
- We use the multi-index as follows. For $x=\left(x_{1}, \ldots, x_{n}\right), y=\left(y_{1}, \ldots, y_{n}\right) \in$ $\mathbb{R}^{n}, \alpha=\left(\alpha_{1}, \ldots, \alpha_{n}\right) \in \mathbb{Z}_{+}^{n}, p=\left(p_{1}, \ldots, p_{n}\right) \in \mathbb{N}^{n}$, define

$$
\begin{aligned}
& |x|=\sqrt{\left|x_{1}\right|^{2}+\cdots+\left|x_{n}\right|^{2}}, \quad\langle x, y\rangle=x_{1} y_{1}+\cdots+x_{n} y_{n}, \\
& x^{\alpha}=x_{1}^{\alpha_{1}} \cdots x_{n}^{\alpha_{n}}, \quad \partial^{\alpha}=\left(\frac{\partial}{\partial x_{1}}\right)^{\alpha_{1}} \cdots\left(\frac{\partial}{\partial x_{n}}\right)^{\alpha_{n}}, \\
& \langle\alpha\rangle=\alpha_{1}+\cdots+\alpha_{n}, \quad \alpha !=\alpha_{1} ! \cdots \alpha_{n} !, \quad 0 !=1, \\
& \alpha / p=\left(\alpha_{1} / p_{1}, \ldots, \alpha_{n} / p_{n}\right), \quad x^{\alpha / p}=x_{1}^{\alpha_{1} / p_{1}} \cdots x_{n}^{\alpha_{n} / p_{n}} .
\end{aligned}
$$

- For $A, B \subset \mathbb{R}^{n}$ and $c \in \mathbb{R}$, we set

$A+B=\left\{a+b \in \mathbb{R}^{n}: a \in A\right.$ and $\left.b \in B\right\}, \quad c \cdot A=\left\{c a \in \mathbb{R}^{n}: a \in A\right\}$.

Moreover, $\operatorname{Int}(A)$ expresses the interior of the set $A$.

- We express by 1 the vector $(1, \ldots, 1)$ or the set $\{(1, \ldots, 1)\}$. For $x=$ $\left(x_{1}, \ldots, x_{n}\right) \in \mathbb{R}^{n}, x^{\mathbf{1}}$ means $x_{1} \cdots x_{n}$.

- For a finite set $A, \# A$ means the cardinality of $A$.

- For a nonnegative real number $r$ and a subset $I$ in $\{1, \ldots, n\}$, the map $T_{I}^{r}: \mathbb{R}^{n} \rightarrow \mathbb{R}^{n}$ is defined by

$$
\left(z_{1}, \ldots, z_{n}\right)=T_{I}^{r}\left(x_{1}, \ldots, x_{n}\right) \text { with } z_{j}:= \begin{cases}r & \text { for } j \in I, \\ x_{j} & \text { otherwise. }\end{cases}
$$

We define $T_{I}:=T_{I}^{0}$. For a set $A$ in $\mathbb{R}^{n}$, the image of $A$ by $T_{I}$ is denoted by $T_{I}(A)$. When $A=\mathbb{R}^{n}$ or $\mathbb{Z}_{+}^{n}$, its image is expressed as

$$
T_{I}(A)=\left\{x \in A: x_{j}=0 \text { for } j \in I\right\} .
$$

- For a smooth function $f$, we denote by $\operatorname{Supp}(f)$ the support of $f$, i.e., $\operatorname{Supp}(f)$ is the closure of the set $\left\{x \in \mathbb{R}^{n}: f(x) \neq 0\right\}$.

\section{NeWton POLYHEDRA AND THE Class $\hat{\mathcal{E}}(U)$}

2.1. Polyhedra. Let us explain fundamental notions in the theory of convex polyhedra which are necessary for our investigation. Refer to 32 for a general theory of convex polyhedra.

For $(a, l) \in \mathbb{R}^{n} \times \mathbb{R}$, let $H(a, l)$ and $H^{+}(a, l)$ be a hyperplane and a closed halfspace in $\mathbb{R}^{n}$ defined by

$$
\begin{aligned}
& H(a, l):=\left\{x \in \mathbb{R}^{n}:\langle a, x\rangle=l\right\}, \\
& H^{+}(a, l):=\left\{x \in \mathbb{R}^{n}:\langle a, x\rangle \geq l\right\},
\end{aligned}
$$

respectively. A (convex rational) polyhedron is an intersection of closed halfspaces: a set $P \subset \mathbb{R}^{n}$ presented in the form $P=\bigcap_{j=1}^{N} H^{+}\left(a^{j}, l_{j}\right)$ for some $a^{1}, \ldots, a^{N} \in \mathbb{Z}^{n}$ and $l_{1}, \ldots, l_{N} \in \mathbb{Z}$.

Let $P$ be a polyhedron in $\mathbb{R}^{n}$. A pair $(a, l) \in \mathbb{Z}^{n} \times \mathbb{Z}$ is said to be valid for $P$ if $P$ is contained in $H^{+}(a, l)$. A face of $P$ is any set of the form $F=P \cap H(a, l)$, where $(a, l)$ is valid for $P$. Since $(0,0)$ is always valid, we consider $P$ itself as a trivial face of $P$; the other faces are called proper faces. Conversely, it is easy to see that any face is a polyhedron. Considering the valid pair $(0,-1)$, we see that the empty set is always a face of $P$. Indeed, $H^{+}(0,-1)=\mathbb{R}^{n}$, but $H(0,-1)=\emptyset$. We write

$\mathcal{F}[P]=$ the set of all nonempty faces of $P$. 
The dimension of a face $F$ is the dimension of its affine hull (i.e., the intersection of all affine flats that contain $F$ ), which is denoted by $\operatorname{dim}(F)$. The faces of dimensions 0,1 and $\operatorname{dim}(P)-1$ are called vertices, edges and facets, respectively. The boundary of a polyhedron $P$, denoted by $\partial P$, is the union of all proper faces of $P$. For a face $F, \partial F$ is similarly defined.

Remark 2.1. It follows from the definition of $H^{+}(\cdot, \cdot)$ that for $a \in \mathbb{Z}^{n}, l \in \mathbb{R}$,

$$
\begin{aligned}
& H^{+}(a, d l)=d \cdot H^{+}(a, l) \text { for } d>0, \\
& H^{+}(a, l+\langle a, b\rangle)=H^{+}(a, l)+b \text { for } b \in \mathbb{Z}^{n} .
\end{aligned}
$$

In the case of hyperplanes $H(a, l)$, analogous equations can be obtained.

Every polyhedron treated in this paper satisfies a condition in the following lemma.

Lemma 2.2. Let $P \subset \mathbb{R}_{+}^{n}$ be a polyhedron. Then the following conditions are equivalent.

(i) $P+\mathbb{R}_{+}^{n} \subset P$.

(ii) There exists a finite set of pairs $\left\{\left(a^{j}, l_{j}\right)\right\}_{j=1}^{N} \subset \mathbb{Z}_{+}^{n} \times \mathbb{Z}_{+}$such that $P=$ $\bigcap_{j=1}^{N} H^{+}\left(a^{j}, l_{j}\right)$.

Proof. (i) $\Longrightarrow$ (ii). Suppose that (ii) does not hold. From the definition of the polyhedron, $P$ is expressed as $P=\bigcap_{j=1}^{N} H^{+}\left(a^{j}, l_{j}\right)$ with $\left(a^{j}, l_{j}\right) \in \mathbb{Z}^{n} \times \mathbb{Z}$. Here, it may be assumed that the set $A:=\left\{\left(a^{j}, l_{j}\right)\right\}_{j=1}^{N}$ satisfies that $P \cap H\left(a^{j}, l_{j}\right) \neq \emptyset$ for all $j$. If $(a, l) \in A$ belongs to $\mathbb{Z}_{+}^{n} \times(-\mathbb{N})$, then $P \cap H(a, l)=\emptyset$. On the other hand, if there exists $(a, l) \in A$ with $a \in \mathbb{Z}^{n} \backslash \mathbb{Z}_{+}^{n}$, then the nonempty face $\gamma:=P \cap H(a, l)$ satisfies $\gamma+\mathbb{R}_{+}^{n} \not \subset H^{+}(a, l)$, which implies $P+\mathbb{R}_{+}^{n} \not \subset P$.

(ii) $\Longrightarrow$ (i). This implication easily follows from the following: For any $(a, l) \in$ $\mathbb{Z}_{+}^{n} \times \mathbb{Z}_{+}$, if $\alpha \in H^{+}(a, l)$, then $\alpha+\mathbb{R}_{+}^{n} \subset H^{+}(a, l)$.

2.2. Newton polyhedra. Let us define the Newton polyhedron and some important classes of smooth functions characterized in terms of the Newton polyhedron.

Let $f$ be a smooth function defined on a neighborhood of the origin in $\mathbb{R}^{n}$, which has the Taylor series at the origin:

$$
f(x) \sim \sum_{\alpha \in \mathbb{Z}_{+}^{n}} c_{\alpha} x^{\alpha} \quad \text { with } c_{\alpha}=\frac{\partial^{\alpha} f(0)}{\alpha !} .
$$

Definition 2.3. The Newton polyhedron $\Gamma_{+}(f)$ of $f$ is defined to be the convex hull of the set $\bigcup\left\{\alpha+\mathbb{R}_{+}^{n}: c_{\alpha} \neq 0\right\}$.

It is known that the Newton polyhedron is a polyhedron (see [32]). The union of the compact faces of the Newton polyhedron $\Gamma_{+}(f)$ is called the Newton diagram $\Gamma(f)$ of $f$, while the topological boundary of $\Gamma_{+}(f)$ is denoted by $\partial \Gamma_{+}(f)$. The polynomial

$$
f_{\Gamma(f)}(x):=\sum_{\alpha \in \Gamma(f) \cap \mathbb{Z}_{+}^{n}} c_{\alpha} x^{\alpha}
$$


is an important part of the series (2.3). The following classes of smooth functions often appear in this paper.

- $f$ is said to be flat if $\Gamma_{+}(f)=\emptyset$ (i.e., all derivatives of $f$ vanish at the origin).

- $f$ is said to be convenient if the Newton polyhedron $\Gamma_{+}(f)$ intersects all the coordinate axes.

2.3. Newton distance and multiplicity. Let $f, g$ be nonflat smooth functions defined on a neighborhood of the origin in $\mathbb{R}^{n}$. We define the Newton distance and the Newton multiplicity with respect to the pair $(f, g)$. At the same time, consider important faces of $\Gamma_{+}(f)$ and $\Gamma_{+}(g)$, which will initially affect the analysis of oscillatory integrals. Hereafter, we assume that $f(0)=0$.

Definition 2.4. The Newton distance of the pair $(f, g)$ is defined by

$$
d(f, g):=\max \left\{d>0: \partial \Gamma_{+}(f) \cap d \cdot\left(\Gamma_{+}(g)+\mathbf{1}\right) \neq \emptyset\right\} .
$$

This distance will be crucial to determine or estimate the oscillation index.

Remark 2.5. It is easy to see the following.

- $d(f, g)=\min \left\{d>0: d \cdot\left(\Gamma_{+}(g)+\mathbf{1}\right) \subset \Gamma_{+}(f)\right\}$.

- $d(f, g)=\max \left\{d\left(f, x^{\beta}\right): \beta \in \Gamma_{+}(g)\right\}$.

- $d(f, g) \leq \min \left\{d\left(x^{\alpha}, g\right): \alpha \in \Gamma_{+}(f)\right\}$ (notice that " $<$ " is possible).

In [1, p. 254], the number $d(f, g)$ is called the coefficient of inscription of $\Gamma_{+}(g)$ in $\Gamma_{+}(f)$. (In [1], this number is defined by $\min \left\{d>0: d \cdot \Gamma_{+}(g) \subset \Gamma_{+}(f)\right\}$, which must be corrected as in (2.5).)

We define the map $\Phi: \mathbb{R}^{n} \rightarrow \mathbb{R}^{n}$ as

$$
\Phi(\beta):=d(f, g)(\beta+\mathbf{1}) .
$$

The image of $\Gamma_{+}(g)$ by the map $\Phi$ comes in contact with the boundary of $\Gamma_{+}(f)$. We denote by $\Gamma_{0}(f)$ this contacting set on $\partial \Gamma_{+}(f)$ and by $\Gamma_{0}(g)$ the image of $\Gamma_{0}(f)$ by the inverse map of $\Phi$, i.e.,

$$
\begin{aligned}
& \Gamma_{0}(f):=\partial \Gamma_{+}(f) \cap \Phi\left(\Gamma_{+}(g)\right)\left(=\partial \Gamma_{+}(f) \cap d(f, g) \cdot\left(\Gamma_{+}(g)+\mathbf{1}\right)\right) ; \\
& \Gamma_{0}(g):=\Phi^{-1}\left(\Gamma_{0}(f)\right)\left(=\left(\frac{1}{d(f, g)} \cdot \partial \Gamma_{+}(f)-\mathbf{1}\right) \cap \Gamma_{+}(g)\right) .
\end{aligned}
$$

Note that $\Gamma_{0}(g)$ is a certain union of faces of $\Gamma_{+}(g)$. It is easy to see the following.

- The map $\Phi: \mathbb{R}^{n} \rightarrow \mathbb{R}^{n}$ is a bijection and so is its restricted map $\left.\Phi\right|_{\Gamma_{0}(g)}$ : $\Gamma_{0}(g) \rightarrow \Gamma_{0}(f)$.

- If $\beta \in \Gamma_{0}(g)$, then $d(f, g)=d\left(f, x^{\beta}\right)$.

- If $\alpha \in \Gamma_{0}(f)$, then $d(f, g) \leq d\left(x^{\alpha}, g\right)$ (notice that " $<$ " is possible).

Let us define the Newton multiplicity and important faces of $\Gamma_{+}(f)$ and $\Gamma_{+}(g)$, which will play important roles in the investigation of multiplicity of the oscillation index, by using the map

$$
\tau_{f}: \partial \Gamma_{+}(f) \rightarrow \mathcal{F}\left[\Gamma_{+}(f)\right]
$$

defined as follows (see the definition (2.2) of $\mathcal{F}[\cdot]$ ). For $\alpha \in \partial \Gamma_{+}(f)$, let $\tau_{f}(\alpha)$ be the smallest face of $\Gamma_{+}(f)$ containing $\alpha$. In other words, $\tau_{f}(\alpha)$ is the face whose relative interior contains the point $\alpha \in \partial \Gamma_{+}(f)$. Define

$$
\mathcal{F}_{0}\left[\Gamma_{+}(f)\right]:=\left\{\tau_{f}(\alpha) \in \mathcal{F}\left[\Gamma_{+}(f)\right]: \alpha \in \Gamma_{0}(f)\right\} .
$$


Definition 2.6. The Newton multiplicity of the pair $(f, g)$ is defined by

$$
m(f, g):=\max \left\{n-\operatorname{dim}(\tau): \tau \in \mathcal{F}_{0}\left[\Gamma_{+}(f)\right]\right\} .
$$

Remark 2.7. It is easy to see the following.

- $m(f, g)=\max \left\{m\left(f, x^{\beta}\right): \beta \in \Gamma_{0}(g)\right\}$.

- If $\Gamma_{0}(f)$ contains a vertex of $\Gamma_{+}(f)$, then $m(f, g)=n$.

Definition 2.8. Define

$$
\mathcal{F}_{*}\left[\Gamma_{+}(f)\right]:=\left\{\tau \in \mathcal{F}_{0}\left[\Gamma_{+}(f)\right]: n-\operatorname{dim}(\tau)=m(f, g)\right\} .
$$

The elements of the above set are called the principal faces of $\Gamma_{+}(f)$. Define

$$
\mathcal{F}_{*}\left[\Gamma_{+}(g)\right]:=\left\{\Phi^{-1}\left(\tau_{*}\right) \cap \Gamma_{+}(g): \tau_{*} \in \mathcal{F}_{*}\left[\Gamma_{+}(f)\right]\right\} .
$$

It is easy to see that every element of the above set is a face of $\Gamma_{+}(g)$, which is called a principal face of $\Gamma_{+}(g)$. The map $\Psi_{*}: \mathcal{F}_{*}\left[\Gamma_{+}(f)\right] \rightarrow \mathcal{F}_{*}\left[\Gamma_{+}(g)\right]$ is defined as $\Psi_{*}\left(\tau_{*}\right):=\Phi^{-1}\left(\tau_{*}\right) \cap \Gamma_{+}(g)$. It is easy to see that this map is bijective. We say that $\tau_{*} \in \mathcal{F}_{*}\left[\Gamma_{+}(f)\right]$ (resp. $\gamma_{*} \in \mathcal{F}_{*}\left[\Gamma_{+}(g)\right]$ ) is associated to $\gamma_{*} \in \mathcal{F}_{*}\left[\Gamma_{+}(g)\right]$ (resp. $\left.\tau_{*} \in \mathcal{F}_{*}\left[\Gamma_{+}(f)\right]\right)$ if $\gamma_{*}=\Psi_{*}\left(\tau_{*}\right)$.

Remark 2.9. In [5], the union of the faces belonging to $\mathcal{F}_{*}\left[\Gamma_{+}(g)\right]$ was called the essential set on $\Gamma_{0}(g)$. It is shown in [5] that every two faces belonging to $\mathcal{F}_{*}\left[\Gamma_{+}(g)\right]$ are disjoint.

Remark 2.10. It is easy to see the following.

- If $\tau_{*} \in \mathcal{F}_{*}\left[\Gamma_{+}(f)\right]$, then $\Psi_{*}\left(\tau_{*}\right)=\left\{\beta \in \Gamma_{0}(g):\left(\tau_{f} \circ \Phi\right)(\beta)=\tau_{*}\right\}$.

- If $\gamma_{*}=\Psi_{*}\left(\tau_{*}\right) \in \mathcal{F}_{*}\left[\Gamma_{+}(g)\right]$, then $\operatorname{dim}\left(\gamma_{*}\right) \leq \operatorname{dim}\left(\tau_{*}\right)=n-m(f, g)$.

- If $\gamma_{*}=\Psi_{*}\left(\tau_{*}\right) \in \mathcal{F}_{*}\left[\Gamma_{+}(g)\right]$, then the compactness of $\gamma_{*}$ is equivalent to that of $\tau_{*}$.

- If $f$ is convenient, then every principal face of $\Gamma_{+}(g)$ is compact.

Remark 2.11. Let us consider the case $g(0) \neq 0$. Then $\Gamma_{+}(g)=\mathbb{R}_{+}^{n}$. In this case, since $d(f, g)$ and $m(f, g)$ are independent of $g$, we simply denote them by $d(f)$ and $m(f)$, respectively. It is easy to see the following.

- $d(f, g) \leq d(f)$ for general $g$.

- The Newton distance $d(f)$ is determined by the point $q_{*}=(d(f), \ldots, d(f))$, which is the intersection of the line $\alpha_{1}=\cdots=\alpha_{n}$ with $\partial \Gamma_{+}(f)$.

- $\Gamma_{0}(g)$ and the principal face of $\Gamma_{+}(g)$ contain the point $\{0\}$. (Note that they may not equal $\{0\}$.)

- The principal face of $\Gamma_{+}(f)$ is the smallest face $\tau_{*}$ of $\Gamma_{+}(f)$ containing the point $q_{*}$.

- $m(f)=n-\operatorname{dim}\left(\tau_{*}\right)$.

More generally, in the case when $\Gamma_{+}(g)=\{p\}+\mathbb{R}_{+}^{n}$ with $p \in \mathbb{Z}_{+}^{n}$, the geometrical meanings of the quantities $d(f, g)$ and $m(f, g)$ will be considered in Lemmas 10.4 and 10.5, below.

2.4. The $\gamma$-part. Let $f$ be a $\left(C^{\infty}\right)$ smooth function defined on a neighborhood $V$ of the origin whose Taylor series at the origin is as in (2.3),$P \subset \mathbb{R}_{+}^{n}$ a nonempty polyhedron in $\mathbb{R}_{+}^{n}$ containing $\Gamma_{+}(f)$ and $\gamma$ a face of $P$. Note that $P$ satisfies the condition $P+\mathbb{R}_{+}^{n} \subset P$ (see Lemma 2.2). 
Definition 2.12. We say that $f$ admits the $\gamma$-part on an open neighborhood $U \subset V$ of the origin if for any $x$ in $U$ the limit

$$
\lim _{t \rightarrow 0} \frac{f\left(t^{a_{1}} x_{1}, \ldots, t^{a_{n}} x_{n}\right)}{t^{l}}
$$

exists for all valid pairs $(a, l)=\left(\left(a_{1}, \ldots, a_{n}\right), l\right) \in \mathbb{Z}_{+}^{n} \times \mathbb{Z}_{+}$defining $\gamma$. When $f$ admits the $\gamma$-part, it is known in [20, Proposition 5.2 (iii)], that the above limits take the same value for any $(a, l)$, which is denoted by $f_{\gamma}(x)$. We consider $f_{\gamma}$ as a function on $U$, which is called the $\gamma$-part of $f$ on $U$.

Remark 2.13. From the condition $P+\mathbb{R}_{+}^{n} \subset P$, Lemma 2.2 implies that it is sufficient to consider valid pairs $(a, l)$ belonging to " $\mathbb{Z}_{+}^{n} \times \mathbb{Z}_{+}$".

Remark 2.14. We summarize important properties of the $\gamma$-part. See [20] for the details.

(i) The $\gamma$-part $f_{\gamma}$ is a $C^{\infty}$ smooth function defined on $U$.

(ii) If $f$ admits the $\gamma$-part $f_{\gamma}$ on $U$, then $f_{\gamma}$ has the quasihomogeneous property

$$
f_{\gamma}\left(t^{a_{1}} x_{1}, \ldots, t^{a_{n}} x_{n}\right)=t^{l} f_{\gamma}(x) \text { for } 0<t<1 \text { and } x \in U,
$$

where $(a, l) \in \mathbb{Z}_{+}^{n} \times \mathbb{Z}_{+}$is a valid pair defining $\gamma$.

(iii) For a compact face $\gamma$ of $\Gamma_{+}(f), f$ always admits the $\gamma$-part near the origin and $f_{\gamma}(x)$ equals the polynomial $\sum_{\alpha \in \gamma \cap \mathbb{Z}_{+}^{n}} c_{\alpha} x^{\alpha}$, which is the same as the well-known $\gamma$-part of $f$ in 30, 1 . Note that $\gamma$ is a compact face if and only if every valid pair $(a, l)=\left(a_{1}, \ldots, a_{n}\right)$ defining $\gamma$ satisfies $a_{j}>0$ for any $j$.

(iv) Let $f$ be a smooth function and $\gamma$ a noncompact face of $\Gamma_{+}(f)$. Then, $f$ does not admit the $\gamma$-part in general (see Section 2.6). If $f$ admits the $\gamma$ part, then the Taylor series of $f_{\gamma}(x)$ at the origin is $\sum_{\alpha \in \gamma \cap \mathbb{Z}_{+}^{n}} c_{\alpha} x^{\alpha}$, where the Taylor series of $f$ is as in (2.3).

(v) Let $f$ be a smooth function and $\gamma$ a face defined by the intersection of $\Gamma_{+}(f)$ and some coordinate hyperplane. More exactly, there exists a nonempty subset $I \subset\{1, \ldots, n\}$ such that $\gamma=\Gamma_{+}(f) \cap T_{I}\left(\mathbb{R}_{+}^{n}\right)$ (see (1.5)). Altough $\gamma$ is a noncompact face if $\gamma \neq \emptyset, f$ always admits the $\gamma$-part. Indeed, $T_{I}\left(\mathbb{R}_{+}^{n}\right)$ can be regarded as a face of $\mathbb{R}_{+}^{n}$, which is expressed by $\mathbb{R}_{+}^{n} \cap H\left(a_{I}, 0\right)$, where $a_{I}=\left(a_{1}, \ldots, a_{n}\right) \in \mathbb{R}_{+}^{n}$ satisfies that $a_{j}>0$ iff $j \in I$. Thus every valid pair defining $\gamma$ takes the form $(a, l)=\left(a_{I}, 0\right)$, so $l=0$ implies the existence of the limit (2.12) (see Section 2.6.2, below).

(vi) If $f$ is real analytic and $\gamma$ is a face of $\Gamma_{+}(f)$, then $f$ admits the $\gamma$-part. Moreover, $f_{\gamma}(x)$ is real analytic and is equal to a convergent power series $\sum_{\alpha \in \gamma \cap \mathbb{Z}_{+}^{n}} c_{\alpha} x^{\alpha}$ on some neighborhood of the origin.

Remark 2.15. The $\gamma$-part $f_{\gamma}$ can be uniquely extended to be the smooth function defined on a much wider region $U \cup\left\{x \in \mathbb{R}^{n}:\left|x_{j}\right|<\delta\right.$ for $\left.j \in V(\gamma)\right\}$ with the property (2.13) for $t \in \mathbb{R}$, where $\delta$ is a positive number and $V(\gamma):=\left\{j: a_{j}=0\right\}$. Here $(a, l)$ is a valid pair defining $\gamma$. In particular, if $\gamma$ is compact $(\Leftrightarrow V(\gamma)=\emptyset)$, then $f_{\gamma}$ becomes a polynomial and is defined on the whole space $\mathbb{R}^{n}$. Note that $V(\gamma)$ is independent of the selection of valid pairs. Hereafter, this extended function will also be denoted by $f_{\gamma}$. 
2.5. The classes $\hat{\mathcal{E}}[P](U)$ and $\hat{\mathcal{E}}(U)$. Let $P$ be a polyhedron (possibly an empty set) in $\mathbb{R}^{n}$ satisfying $P+\mathbb{R}_{+}^{n} \subset P$ when $P \neq \emptyset$. Let $U$ be an open neighborhood of the origin.

Definition 2.16. Denote by $\mathcal{E}[P](U)$ the set of smooth functions on $U$ whose Newton polyhedra are contained in $P$. Moreover, when $P \neq \emptyset$, we denote by $\hat{\mathcal{E}}[P](U)$ the set of the elements $f$ in $\mathcal{E}[P](U)$ such that $f$ admits the $\gamma$-part on some neighborhood of the origin for any face $\gamma$ of $P$. When $P=\emptyset, \hat{\mathcal{E}}[P](U)$ is defined to be the set $\{0\}$, i.e., the set consisting of only the function identically equaling zero on $U$.

We summarize properties of the classes $\mathcal{E}[P](U)$ and $\hat{\mathcal{E}}[P](U)$, which can be directly seen from their definitions:

(i) $\hat{\mathcal{E}}\left[\mathbb{R}_{+}^{n}\right](U)=\mathcal{E}\left[\mathbb{R}_{+}^{n}\right](U)=C^{\infty}(U)$.

(ii) If $P_{1}, P_{2} \subset \mathbb{R}_{+}^{n}$ are polyhedra with $P_{1} \subset P_{2}$, then $\mathcal{E}\left[P_{1}\right](U) \subset \mathcal{E}\left[P_{2}\right](U)$ and $\hat{\mathcal{E}}\left[P_{1}\right](U) \subset \hat{\mathcal{E}}\left[P_{2}\right](U)$.

(iii) $\left(C^{\omega}(U) \cap \mathcal{E}[P](U)\right) \subsetneq \hat{\mathcal{E}}[P](U) \subsetneq \mathcal{E}[P](U)$.

(iv) $\mathcal{E}[P](U)$ and $\hat{\mathcal{E}}[P](U)$ are $C^{\infty}(U)$-modules and ideals of $C^{\infty}(U)$.

The following is an important property of the class $\hat{\mathcal{E}}[P](U)$.

Proposition $2.17([20])$. If $P$ is a nonempty polyhedron, then the following conditions are equivalent.

(i) $f$ belongs to the class $\hat{\mathcal{E}}[P](U)$.

(ii) There exist a finite set $S$ in $P \cap \mathbb{Z}_{+}^{n}$ and $\psi_{p} \in C^{\infty}(U)$ for $p \in S$ such that

$$
f(x)=\sum_{p \in S} x^{p} \psi_{p}(x) .
$$

Note that the above expression is not unique.

Definition 2.18. $\hat{\mathcal{E}}(U):=\left\{f \in C^{\infty}(U): f \in \hat{\mathcal{E}}\left[\Gamma_{+}(f)\right](U)\right\}$.

It is easy to see the following properties of the class $\hat{\mathcal{E}}(U)$.

(i) $C^{\omega}(U) \subsetneq \hat{\mathcal{E}}(U) \subsetneq C^{\infty}(U)$.

(ii) When $f$ is flat but $f \not \equiv 0, f$ does not belong to $\hat{\mathcal{E}}(U)$.

The class $\hat{\mathcal{E}}(U)$ contains many kinds of smooth functions.

- $\hat{\mathcal{E}}(U)$ contains the function identically equaling zero on $U$.

- Every real analytic function defined on $U$ belongs to $\hat{\mathcal{E}}(U)$. (From (vi) in Remark 2.14)

- If $f \in C^{\infty}(U)$ is convenient, then $f$ belongs to $\hat{\mathcal{E}}(U)$. (In this case, every proper noncompact face of $\Gamma_{+}(f)$ can be expressed by the intersection of $\Gamma_{+}(f)$ and some coordinate hyperplane. Therefore, (iii), (v) in Remark 2.14 imply this assertion.)

- In the one-dimensional case, every nonflat smooth function belongs to $\hat{\mathcal{E}}(U)$. (This is a particular case of the above convenient case.)

- The Denjoy-Carleman (quasianalytic) classes are contained in $\hat{\mathcal{E}}(U)$. (See Proposition 6.10 in [20.) 
Unfortunately, the algebraic structure of $\hat{\mathcal{E}}(U)$ is poor. Indeed, it is not closed under addition. For example, consider $f_{1}\left(x_{1}, x_{2}\right)=x_{1}+x_{1} \exp \left(-1 / x_{2}^{2}\right)$ and $f_{2}\left(x_{1}, x_{2}\right)$ $=-x_{1}$. Indeed, both $f_{1}$ and $f_{2}$ belong to $\hat{\mathcal{E}}(U)$, but $f_{1}+f_{2}\left(=\exp \left(-1 / x_{2}^{2}\right)\right)$ does not belong to $\hat{\mathcal{E}}(U)$.

\subsection{Examples.}

2.6.1. Example 1. In order to show what kinds of functions belong to the classes $\hat{\mathcal{E}}(U), \hat{\mathcal{E}}[P](U)$, let us consider the following two-dimensional example, which lives near the border of these classes:

$$
\begin{aligned}
& f_{k}(x)=f_{k}\left(x_{1}, x_{2}\right):=x_{1}^{2} x_{2}^{2}+x_{1}^{k} e^{-1 / x_{2}^{2}}, \quad k \in \mathbb{Z}_{+} ; \\
& P=\left\{\left(\alpha_{1}, \alpha_{2}\right) \in \mathbb{R}_{+}^{2}: \alpha_{1} \geq 1, \alpha_{2} \geq 1\right\} .
\end{aligned}
$$

Of course, $f_{k}$ is not real analytic around the origin. The set of the proper faces of $\Gamma_{+}\left(f_{k}\right)$ and $P$ consists of $\gamma_{1}, \gamma_{2}, \gamma_{3}$ and $\tau_{1}, \tau_{2}, \tau_{3}$, where

$$
\begin{aligned}
& \gamma_{1}=\left\{\left(2, \alpha_{2}\right): \alpha_{2} \geq 2\right\}, \gamma_{2}=\{(2,2)\}, \gamma_{3}=\left\{\left(\alpha_{1}, 2\right): \alpha_{1} \geq 2\right\} ; \\
& \tau_{1}=\left\{\left(1, \alpha_{2}\right): \alpha_{2} \geq 1\right\}, \tau_{2}=\{(1,1)\}, \tau_{3}=\left\{\left(\alpha_{1}, 1\right): \alpha_{1} \geq 1\right\} .
\end{aligned}
$$

It is easy to see that if $j=2,3$, then $f_{k}$ admits the $\gamma_{j}$-part and $\tau_{j}$-part near the origin for all $k \in \mathbb{Z}_{+}$and they are written as $\left(f_{k}\right)_{\gamma_{2}}(x)=\left(f_{k}\right)_{\gamma_{3}}(x)=x_{1}^{2} x_{2}^{2}$ and $\left(f_{k}\right)_{\tau_{2}}(x)=\left(f_{k}\right)_{\tau_{3}}(x) \equiv 0$. Consider the $\gamma_{1}$-part and $\tau_{1}$-part of $f_{k}$ for $k \in \mathbb{Z}_{+}$. The situation depends on the parameter $k$ as follows.

- $\left(f_{0}\right)_{\gamma_{1}}$ and $\left(f_{0}\right)_{\tau_{1}}$ cannot be defined.

- $\left(f_{1}\right)_{\gamma_{1}}$ cannot be defined, but $\left(f_{1}\right)_{\tau_{1}}(x)=x_{1} e^{-1 / x_{2}^{2}}$.

- $\left(f_{2}\right)_{\gamma_{1}}(x)=f_{2}(x)$ and $\left(f_{2}\right)_{\tau_{1}}(x) \equiv 0$.

- If $k \geq 3$, then $\left(f_{k}\right)_{\gamma_{1}}(x)=x_{1}^{2} x_{2}^{2}$ and $\left(f_{k}\right)_{\tau_{1}}(x) \equiv 0$.

From the above, we see that $f_{k} \in \hat{\mathcal{E}}(U)$ if and only if $k \geq 2 ; f_{k} \in \hat{\mathcal{E}}[P](U)$ if and only if $k \geq 1$. Notice that $\tau_{1} \cap \Gamma_{+}\left(f_{1}\right)=\emptyset$ but $\left(f_{1}\right)_{\tau_{1}}(x)=x_{1} e^{-1 / x_{2}^{2}} \not \equiv 0$.

2.6.2. Example 2. Let $f$ be a smooth function defined near the origin in $\mathbb{R}^{3}$. As mentioned in Remark $2.14(\mathrm{v})$, when $\gamma$ is a face defined by the intersection of $\Gamma_{+}(f)$ and some coordinate hyperplane, $f$ always admits the $\gamma$-part. But, the condition that $\gamma$ is a noncompact face of $\Gamma_{+}(f)$ contained in some coordinate hyperplane is not sufficient for the admission of the $\gamma$-part. Indeed, the following three-dimensional example shows this subtle situation:

$$
f\left(x_{1}, x_{2}, x_{3}\right)=x_{1}^{2}+e^{-1 / x_{2}^{2}} \text { and } \gamma=\left\{\left(2, \alpha_{2}, 0\right): \alpha_{2} \geq 0\right\} .
$$

It is easy to check that the valid pair $((1,0,1), 2)$ defines the face $\gamma$, but the limit (2.12) does not exist when $x_{2} \neq 0$. This implies that the above $f$ does not belong to the class $\hat{\mathcal{E}}(U)$.

\section{EARLIER STUDIES}

As mentioned in the Introduction, there are many kinds of generalizations of the work of Varchenko in 30 relating to the behavior of the oscillatory integral $I(t ; \varphi)$ in (1.1). In order to reveal a motivation of our investigation, after stating results of Varchenko, we explain some earlier results in [30, 31, [1, 26, ,5], 20, which are deeply connected with our study. Note that some conditions in the assumptions of the previous results can be considered as typical cases of the assumptions in our 
new theorems in Section 4, so they are sometimes more useful for the practical applications.

Throughout this section, the following three conditions are assumed: Let $U$ be an open neighborhood of the origin in $\mathbb{R}^{n}$.
(A) $f$ is a nonflat smooth function defined on $U$ satisfying that $f(0)=|\nabla f(0)|$ $=0$
(B) $g$ is a nonflat smooth function defined on $U$;
(C) $\varphi$ is a smooth function whose support is contained in $U$.

3.1. Results of Varchenko. Let us recall a part of the famous results due to Varchenko in [30] and Arnold, Gusein-Zade and Varchenko [1] in the case when $f$ is real analytic on $U$ and $g \equiv 1$. These results require the following condition.

(D) $f$ is real analytic on $U$ and is nondegenerate over $\mathbb{R}$ with respect to the Newton polyhedron $\Gamma_{+}(f)$; i.e., for every compact face $\gamma$ of $\Gamma_{+}(f)$, the $\gamma$-part $f_{\gamma}$ satisfies

$$
\nabla f_{\gamma}=\left(\frac{\partial f_{\gamma}}{\partial x_{1}}, \ldots, \frac{\partial f_{\gamma}}{\partial x_{n}}\right) \neq(0, \ldots, 0) \text { on the set }(\mathbb{R} \backslash\{0\})^{n} .
$$

Theorem 3.1 ([30], [1]). If $f$ satisfies the condition (D), then the following hold:

(i) The progression $\{\alpha\}$ in (1.2) belongs to finitely many arithmetic progressions, which are obtained by using the theory of toric varieties based on the geometry of the Newton polyhedron $\Gamma_{+}(f)$. (See Remark 4.2, below.)

(ii) $\beta(f, 1) \leq-1 / d(f)$.

(iii) If at least one of the following conditions is satisfied:

(a) $d(f)>1$

(b) $f$ is nonnegative or nonpositive on $U$;

(c) $1 / d(f)$ is not an odd integer and $f_{\tau_{*}}$ does not vanish on $U \cap(\mathbb{R} \backslash\{0\})^{n}$, where $\tau_{*}$ is the principal face of $\Gamma_{+}(f)$ (see Remark 2.11),

then $\beta(f, 1)=-1 / d(f)$ and $\eta(f, 1)=m(f)$.

There have been many kinds of studies which generalize or improve the above theorem. Now, let us consider the following two kinds of generalizations of Varchenko's result:

- Consider the weighted case under the real analytic assumption on the phase.

- Weaken the regularity of the phase in the unweighted case.

3.2. Weighted case. The following theorem naturally generalizes the assertion (ii) in Theorem 3.1.

Theorem 3.2 ([5]). Suppose that (i) $f$ satisfies the condition (D) and (ii) at least one of the following conditions is satisfied:

(a) $f$ is convenient;

(b) $g$ is convenient;

(c) $g$ is real analytic on $U$;

(d) $g$ is expressed as $g(x)=x^{p} \tilde{g}(x)$ on $U$, where $p \in \mathbb{Z}_{+}^{n}$ and $\tilde{g}$ is a smooth function defined on $U$ with $\tilde{g}(0) \neq 0$.

Then, we have $\beta(f, g) \leq-1 / d(f, g)$.

The following theorem partially generalizes the assertion (iii) in Theorem 3.1. 
Theorem $3.3([5])$. Suppose that (i) $f$ satisfies the condition (D); (ii) at least one of the following two conditions is satisfied:

(a) $f$ is convenient and $g_{\gamma_{*}}$ is nonnegative or nonpositive on $U$ for all principal faces $\gamma_{*}$ of $\Gamma_{+}(g)$;

(b) $g$ is expressed as $g(x)=x^{p} \tilde{g}(x)$ on $U$, where every component of $p \in \mathbb{Z}_{+}^{n}$ is even and $\tilde{g}$ is a smooth function defined on $U$ with $\tilde{g}(0) \neq 0$;

and (iii) at least one of the following two conditions is satisfied:

(c) $d(f, g)>1$;

(d) $f$ is nonnegative or nonpositive on $U$.

Then the equations $\beta(f, g)=-1 / d(f, g)$ and $\eta(f, g)=m(f, g)$ hold.

Remark 3.4. Similar results to the above two theorems have been obtained in [31, [1. In our language, the results in [1] can be stated as follows.

(Theorem 8.4 in [1, p. 254]) If $f$ is real analytic and is nondegenerate over $\mathbb{R}$ with respect to its Newton polyhedron, then

(i) $\beta(f, g) \leq-1 / d(f, g)$.

(ii) If $d(f, g)>1$ and $\Gamma_{+}(g)=\{p\}+\mathbb{R}_{+}^{n}$ with $p \in \mathbb{Z}_{+}^{n}$, then $\beta(f, g)=$ $-1 / d(f, g)$.

Unfortunately, more additional assumptions are necessary to obtain the above assertions (i), (ii). Indeed, consider the following two-dimensional example:

$$
f\left(x_{1}, x_{2}\right)=x_{1}^{4} ; \quad g\left(x_{1}, x_{2}\right)=x_{1}^{2} x_{2}^{2}+e^{-1 / x_{2}^{2}},
$$

which is a special case of the example in Section 15.1. It follows from the computation in Section 15.1 that this example violates (i), (ii). As for (ii), even if $g$ is real analytic, the one-dimensional case indicates that at least some condition on the power $p$ is needed. Indeed, consider the case that $f(x)=x^{2}$ and $g(x)=x^{p}$ where $p$ is an odd positive integer. In this case, the coefficient of the first candidate term $t^{-(p+1) / 2}$ of the asymptotic expansion (1.2) vanishes (see [5, Section 7.4] for the details). It is easy to find counterexamples in the higher dimensional case.

Remark 3.5. Pramanik and Yang [26] obtained a similar result relating to the above equation " $\beta(f, g)=-1 / d(f, g)$ " in the case when the dimension is two and the weight has the form $g(x)=|h(x)|^{\epsilon}$ where $h$ is real analytic and $\epsilon$ is positive. Their approach is based on the Puiseux series expansions of the roots of $f$ and $h$, which is inspired by the work of Phong and Stein in 25]. Their definition of Newton distance, which is different from ours, is given through the process of a good choice of coordinate system. As a result, their result does not need the nondegeneracy condition of the phase.

The following theorem shows an interesting "symmetry property" with respect to the phase and the weight.

Theorem 3.6 ([5]). Suppose that $f, g$ satisfy the condition (D) and that they are convenient and nonnegative or nonpositive on $U$. Then we have $\beta\left(x^{\mathbf{1}} f, g\right) \beta\left(x^{\mathbf{1}} g, f\right)$ $\geq 1$, where $x^{\mathbf{1}}=x_{1} \cdots x_{n}$. Moreover, the following two conditions are equivalent:

(i) $\beta\left(x^{\mathbf{1}} f, g\right) \beta\left(x^{\mathbf{1}} g, f\right)=1$.

(ii) There exists a positive rational number $d$ such that $\Gamma_{+}\left(x^{\mathbf{1}} f\right)=d \cdot \Gamma_{+}\left(x^{\mathbf{1}} g\right)$. If condition (i) or (ii) is satisfied, then we have $\eta\left(x^{\mathbf{1}} f, g\right)=\eta\left(x^{\mathbf{1}} g, f\right)=n$. 
3.3. Weakened regularity of the phase. Let us consider the case when the phase satisfies a weaker regularity condition:

(E) $f$ belongs to the class $\hat{\mathcal{E}}(U)$ and is nondegenerate over $\mathbb{R}$ with respect to its Newton polyhedron.

It is known in 20] that $I(t ; \varphi)$ also has an asymptotic expansion in the case when the phase satisfies the above condition. The following theorem generalizes the assertion (i) in Theorem 3.1.

Theorem 3.7 ([20]). If $f$ satisfies the condition (E) and the support of $\varphi$ is contained in a sufficiently small neighborhood of the origin, then $I(t ; \varphi)$ admits an asymptotic expansion of the form (1.2), where $\{\alpha\}$ belongs to the same progressions as in the case when the phase is $f_{\Gamma(f)}$ as in (2.4). (Since $f_{\Gamma(f)}$ is a polynomial, the progressions can be exactly constructed as in [30.)

Furthermore, Varchenko's results can be directly generalized to the case when the phase belongs to the class $\hat{\mathcal{E}}(U)$.

Theorem $3.8([20])$. If $f$ satisfies the condition (E) and $g \equiv 1$, then the assertions (ii), (iii) in Theorem 3.1 hold.

Remark 3.9. In [20], more precise results are obtained.

Remark 3.10. Some kind of restriction to the regularity of the phase is necessary in the above theorem. Consider the following two-dimensional example: $f\left(x_{1}, x_{2}\right)=$ $x_{1}^{2}+e^{-1 /\left|x_{2}\right|^{\alpha}}(\alpha>0)$ and $g \equiv 1$, which is given by Iosevich and Sawyer in [17]. Note that the above $f$ satisfies the nondegeneracy condition (3.1), but it does not belong to $\hat{\mathcal{E}}(U)$. It is easy to see the following: $d(f)=2, m(f)=1, f_{\tau_{*}}\left(x_{1}, x_{2}\right)=x_{1}^{2}$. It was shown in [17] that $|I(t ; \varphi)| \leq C t^{-1 / 2}(\log t)^{-1 / \alpha}$ for $t \geq 2$. In particular, we have $\lim _{t \rightarrow \infty} t^{1 / 2} I(t ; \varphi)=0$. The pattern of an asymptotic expansion of $I(t ; \varphi)$ in this example might be different from that in (1.2).

\section{MAin Results}

By mixing two kinds of generalizations in the previous section and deeper understanding of the resolution of singularities for the phase and the weight, we can generalize and improve the results in [5]. Furthermore, the results can be stated in more clear form by using the class $\hat{\mathcal{E}}(U)$. This means that properties of $\hat{\mathcal{E}}(U)$ play crucial roles in the sufficient condition on the phase and the weight. Throughout this section, the three conditions (A), (B), (C) at the beginning of Section 3 are assumed, where $U$ is an open neighborhood of the origin in $\mathbb{R}^{n}$.

First, let us give a sharp estimate for $I(t ; \varphi)$. Since the class $\hat{\mathcal{E}}(U)$ contains many kinds of smooth functions as in Section 2.5, the following theorem generalizes and improves Theorem 3.2 in Section 3.

Theorem 4.1. Suppose that (i) $f$ satisfies the condition (E) (see Section 3.3), and (ii) at least one of the following two conditions is satisfied:

(a) $g$ belongs to the class $\hat{\mathcal{E}}(U)$;

(b) $f$ is convenient. 
If the support of $\varphi$ is contained in a sufficiently small neighborhood of the origin, then there exists a positive constant $C(\varphi)$ independent of $t$ such that

$$
|I(t ; \varphi)| \leq C(\varphi) t^{-1 / d(f, g)}(\log t)^{m(f, g)-1} \quad \text { for } t \geq 2 .
$$

In particular, we have $\beta(f, g) \leq-1 / d(f, g)$.

Remark 4.2. From the proof of the above theorem, we can see that, under the same assumptions, the set $\{\alpha\}$ in the asymptotic expansion (1.2) belongs to the following set:

$$
\left\{-\frac{l_{g}(a)+\langle a\rangle+\nu}{l_{f}(a)}: \nu \in \mathbb{Z}_{+}, a \in \tilde{\Sigma}^{(1)}\right\} \cup(-\mathbb{N}),
$$

where $l_{g}(a), l_{f}(a) \in \mathbb{Z}_{+}$are as in (7.1) and $\tilde{\Sigma}^{(1)}$ is a finite set of vectors in $\mathbb{Z}_{+}^{n}$ defined in Theorem 10.1. Of course, the same assertion holds in the cases of Theorem 3.1 (i) and Theorem 3.7. Note that $l_{g}(a)=0$ for any $a \in \mathbb{Z}_{+}^{n}$ if $g(0) \neq 0$.

Remark 4.3. Let us consider the case when the weight $g$ is expressed as $g(x)=$ $x^{p} \psi(x)$ where $p \in \mathbb{Z}_{+}^{n}$ and $\psi \in C^{\infty}(U)$. (In this case, $g$ does not belong to $\hat{\mathcal{E}}(U)$ if and only if $\psi$ is a nonzero flat function.) Then, without the assumption (ii), the following estimate is obtained under the same assumptions:

$$
|I(t ; \varphi)| \leq C(\varphi) t^{-1 / d\left(f, x^{p}\right)}(\log t)^{m\left(f, x^{p}\right)-1} \quad \text { for } t \geq 2 .
$$

This estimate will be shown in Section14.3 (see also Remark 10.3). Note that these assertions hold even if $\psi$ is a nonzero flat function. In this case, $g$ does not belong to $\hat{\mathcal{E}}(U)$, because $g$ is also a nonzero flat function. When $\psi$ vanishes at the origin in high order (in particular $\psi$ is flat), the reader might feel that the estimate (4.2) is not optimal. But, we will give a simple example showing some kind of optimality of the estimate (4.2) even in the flat case (see Example 1 in Section 15).

Next, let us consider the case when the equality $\beta(f, g)=-1 / d(f, g)$ holds. The following theorem generalizes and improves Theorem 3.3 in Section 3.

Theorem 4.4. Suppose that the conditions (i), (ii) in Theorem 4.1 are satisfied, (iii) there exists a principal face $\gamma_{*}$ of $\Gamma_{+}(g)$ such that $g_{\gamma_{*}}$ is nonnegative or nonpositive on $U$ and (iv) at least one of the following three conditions is satisfied:

(a) $d(f, g)>1$;

(b) $f$ is nonnegative or nonpositive on $U$;

(c) $1 / d(f, g)$ is not an odd integer and $f_{\tau_{*}}$ does not vanish on $U \cap(\mathbb{R} \backslash\{0\})^{n}$ where $\tau_{*}$ is a principal face of $\Gamma_{+}(f)$ associated to $\gamma_{*}$ in (iii).

Then the equations $\beta(f, g)=-1 / d(f, g)$ and $\eta(f, g)=m(f, g)$ hold.

Remark 4.5. In Theorem 14.2 in Section 14, we give explicit formulae for the coefficient of the leading term of the asymptotic expansion (1.2) under the assumptions (i)-(iii). These explicit formulae show that the above coefficient essentially depends on the principal face-parts $f_{\tau_{*}}$ and $g_{\gamma_{*}}$. The above (i)-(iv) are sufficient conditions for the nonvanishing of the leading term.

Remark 4.6. Let us discuss the necessities of the hypotheses (i)-(iii).

(i) As explained in Remark 3.10, some kind of restriction to the regularity of the phase is necessary for the two equalities in the above theorem.

(ii) When $f$ is convenient, the strong assumption on the weight $g$ is no longer needed. Without the convenience of $f$, it is a complex issue to give "good " sufficient conditions on $g$. These problems will be discussed in Section 12.1. Furthermore, 
in Section 15, we give a few examples showing the subtlety of the sufficient condition (a): " $g \in \hat{\mathcal{E}}(U)$ ". Now, let us assume that $f, g$ satisfy all the conditions in Theorem 4.4 except the condition (ii). First, we show some kind of necessity of the condition (a). Example 1 shows the existence of $f$ and $g$ such that $g \notin \hat{\mathcal{E}}(U)$ and the equations $\beta(f, g)=-1 / d(f, g)$ in the theorem do not hold. Next, we show that there is room for improvement on the condition (a). By observing Example 2, it is natural to feel that (a) is too strong and to expect that this condition can be weakened by the condition:

$\left(\mathrm{a}^{\prime}\right) g$ belongs to the class $\hat{\mathcal{E}}\left[\Phi^{-1}\left(\Gamma_{+}(f)\right) \cap \mathbb{R}_{+}^{n}\right](U)$

(see the definition of $\Phi$ in $(\underline{2.6})$ ). Unfortunately, Example 3 violates this expectation.

These kinds of subtle situations, which are seen in the above (i) and (ii), occur from the fact that the geometry of the Newton polyhedra does not provide sufficient analytic information about smooth functions.

(iii) The most interesting condition in (iii) is (c). The necessity of the nonoddness of $1 / d(f, g)$ is shown by means of the example, which was given by Pramanik and Yang [26, Section 6]. Actually, consider the two-dimensional example: $f\left(x_{1}, x_{2}\right)=x_{1} x_{2}, g\left(x_{1}, x_{2}\right)=x_{2}^{2}$. In this case, $d(f, g)=1, f_{\tau_{*}}\left(x_{1}, x_{2}\right)=x_{1} x_{2}$, $g_{\gamma_{*}}\left(x_{1}, x_{2}\right)=x_{2}^{2}$. Though it is easy to see all the other conditions in the assumptions in the above theorem, the oscillation index $\beta(f, g)$ equals $-3(\neq-1 / d(f, g)=-1)$.

Finally, Theorem 3.6 can be generalized in the following form.

Theorem 4.7. Suppose that $f, g$ satisfy the condition (E) and that they are nonnegative or nonpositive on $U$. Then we have $\beta\left(x^{\mathbf{1}} f, g\right) \beta\left(x^{\mathbf{1}} g, f\right) \geq 1$. Moreover, the following two conditions are equivalent:

(i) $\beta\left(x^{\mathbf{1}} f, g\right) \beta\left(x^{\mathbf{1}} g, f\right)=1$.

(ii) There exists a positive rational number $d$ such that $\Gamma_{+}\left(x^{\mathbf{1}} f\right)=d \cdot \Gamma_{+}\left(x^{\mathbf{1}} g\right)$. If the condition (i) or (ii) is satisfied, then we have $\eta\left(x^{\mathbf{1}} f, g\right)=\eta\left(x^{\mathbf{1}} g, f\right)=n$.

Remark 4.8. It is needless to say that the behavior of $I(t ; \varphi)$ is independent of the exchanges of the integral variables. Therefore, if there exists a coordinate in which $f$ and $g$ satisfy the assumptions in each theorem, then the respected assertion holds.

\section{TORIC VARIETIES}

In the analysis of Varchenko in [30], the theory of toric varieties plays a crucial role. Our analysis is also based on this theory. In this section, we recall the method to construct a toric variety from a given fan. Refer to 8 , for the general theory of toric varieties.

5.1. Cones and fans. Let us recall the definitions of important terminology: cone and fan.

A rational polyhedral cone $\sigma \subset \mathbb{R}^{n}$ is a cone generated by finitely many elements of $\mathbb{Z}^{n}$. In other words, there are $u_{1}, \ldots, u_{k} \in \mathbb{Z}^{n}$ such that

$$
\sigma=\left\{\lambda_{1} u_{1}+\cdots+\lambda_{k} u_{k} \in \mathbb{R}^{n}: \lambda_{1}, \ldots, \lambda_{k} \geq 0\right\} .
$$

We say that $\sigma$ is strongly convex if $\sigma \cap(-\sigma)=\{0\}$. By regarding a cone as a polyhedron in $\mathbb{R}^{n}$, the definitions of dimension, face, edge, facet for the cone are given in the same way as in Section 2.1. 
The fan is defined to be a finite collection $\Sigma$ of cones in $\mathbb{R}^{n}$ with the following properties:

- Each $\sigma \in \Sigma$ is a strongly convex rational polyhedral cone.

- If $\sigma \in \Sigma$ and $\tau$ is a face of $\sigma$, then $\tau \in \Sigma$.

- If $\sigma, \tau \in \Sigma$, then $\sigma \cap \tau$ is a face of each.

For a fan $\Sigma$, the union $|\Sigma|:=\bigcup_{\sigma \in \Sigma} \sigma$ is called the support of $\Sigma$. For $k=0,1, \ldots, n$, we denote by $\Sigma^{(k)}$ the set of $k$-dimensional cones in $\Sigma$. The skeleton of a cone $\sigma \in \Sigma$ is the set of all of its primitive integer vectors (i.e., with components relatively prime in $\mathbb{Z}_{+}$) in the edges of $\sigma$. It is clear that the skeleton of $\sigma$ generates $\sigma$ itself and that the number of the elements of the skeleton in $\Sigma^{(k)}$ is not less than $k$. Thus, the set of skeletons of the cones belonging to $\Sigma^{(k)}$ is also expressed by the same symbol $\Sigma^{(k)}$.

It is known (see [8]) that there exists a simplicial subdivision $\tilde{\Sigma}$ of $\Sigma$; that is, $\tilde{\Sigma}$ is a fan satisfying the following properties:

- The fans $\Sigma$ and $\tilde{\Sigma}$ have the same support.

- Each cone of $\tilde{\Sigma}$ lies in some cone of $\Sigma$.

- The skeleton of any cone belonging to $\tilde{\Sigma}$ can be completed to a base of the lattice dual to $\mathbb{Z}^{n}$.

5.2. Construction of toric varieties. Let $\Sigma_{0}$ be a fan satisfying $\left|\Sigma_{0}\right|=\mathbb{R}_{+}^{n}$. Fix a simplicial subdivision $\Sigma$ of $\Sigma_{0}$. For an $n$-dimensional cone $\sigma \in \Sigma$, let $a^{1}(\sigma), \ldots, a^{n}(\sigma)$ be the skeleton of $\sigma$, ordered once and for all. Here, we set the coordinates of the vector $a^{j}(\sigma)$ as

$$
a^{j}(\sigma)=\left(a_{1}^{j}(\sigma), \ldots, a_{n}^{j}(\sigma)\right) .
$$

With every such cone $\sigma$, we associate a copy of $\mathbb{R}^{n}$ which is denoted by $\mathbb{R}^{n}(\sigma)$. We denote by $\pi(\sigma): \mathbb{R}^{n}(\sigma) \rightarrow \mathbb{R}^{n}$ the map defined by $\pi(\sigma)\left(y_{1}, \ldots, y_{n}\right)=\left(x_{1}, \ldots, x_{n}\right)$ with

$$
x_{j}=\prod_{k=1}^{n} y_{k}^{a_{j}^{k}(\sigma)}=y_{1}^{a_{j}^{1}(\sigma)} \cdots y_{n}^{a_{j}^{n}(\sigma)}, \quad j=1, \ldots, n .
$$

We remark that the following conditions are equivalent:

- $a^{1}(\sigma), \ldots, a^{n}(\sigma)$ can be completed to a base of the lattice dual to $\mathbb{Z}^{n}$.

- The inverse map of $\pi(\sigma)$ is rational.

- $\operatorname{det}\left(a_{k}^{j}(\sigma)\right)_{1 \leq j, k \leq n}= \pm 1$.

Let $Y_{\Sigma}$ be the union of $\mathbb{R}^{n}(\sigma)$ for $\sigma$ which are glued along the images of $\pi(\sigma)$. Indeed, for any $n$-dimensional cones $\sigma, \sigma^{\prime} \in \Sigma$, two copies $\mathbb{R}^{n}(\sigma)$ and $\mathbb{R}^{n}\left(\sigma^{\prime}\right)$ can be identified with respect to a rational mapping: $\pi^{-1}\left(\sigma^{\prime}\right) \circ \pi(\sigma): \mathbb{R}^{n}(\sigma) \rightarrow \mathbb{R}^{n}\left(\sigma^{\prime}\right)$ (i.e., $x \in \mathbb{R}^{n}(\sigma)$ and $x^{\prime} \in \mathbb{R}^{n}\left(\sigma^{\prime}\right)$ will coalesce if $\left.\pi^{-1}\left(\sigma^{\prime}\right) \circ \pi(\sigma): x \mapsto x^{\prime}\right)$. Then it is known (see 8 ) that

- $Y_{\Sigma}$ is an $n$-dimensional real algebraic manifold.

- The map $\pi: Y_{\Sigma} \rightarrow \mathbb{R}^{n}$ defined on each $\mathbb{R}^{n}(\sigma)$ as $\pi(\sigma): \mathbb{R}^{n}(\sigma) \rightarrow \mathbb{R}^{n}$ is proper.

- Each $\mathbb{R}^{n}(\sigma)$ is densely embedded in $Y_{\Sigma}$.

The manifold $Y_{\Sigma}$ is called the (real) toric variety associated with $\Sigma$. We call the pair $\left(Y_{\Sigma}, \pi\right)$ the real resolution of singularities associated to $\Sigma$.

The following properties of $\pi(\sigma)$ are useful for the analysis in Sections 8-13. They can be easily seen, so we omit their proofs. 
Lemma 5.1. (i) The set of the points in $\mathbb{R}^{n}(\sigma)$ in which $\pi(\sigma)$ is not an isomorphism is a union of coordinate hyperplanes.

(ii) The Jacobian of the mapping $\pi(\sigma)$ is equal to

$$
J_{\pi(\sigma)}(y)=\epsilon \prod_{j=1}^{n} y_{j}^{\left\langle a^{j}(\sigma)\right\rangle-1},
$$

where $\epsilon$ is 1 or -1 .

\section{Construction of fans from polyhedra}

Let $P \subset \mathbb{R}_{+}^{n}$ be an $n$-dimensional polyhedron satisfying $P+\mathbb{R}_{+}^{n} \subset P$ (see Lemma 2.2 in Section 2). In this section, we explain a method to construct fans from a given polyhedron $P$. Furthermore, we consider precise relationships between cones of this fan and faces of $P$. We denote by $\left(\mathbb{R}^{n}\right)^{\vee}$ the dual space of $\mathbb{R}^{n}$ with respect to the standard inner product.

6.1. Fans associated with polyhedra. For $a=\left(a_{1}, \ldots, a_{n}\right) \in\left(\mathbb{R}^{n}\right)^{\vee}$ with $a_{j} \geq$ 0 , define

$$
l(a)=\min \{\langle a, \alpha\rangle: \alpha \in P\}
$$

and $\gamma(a)=\{\alpha \in P:\langle a, \alpha\rangle=l(a)\}(=H(a, l(a)) \cap P)$. We introduce an equivalence relation $\sim$ in $\left(\mathbb{R}^{n}\right)^{\vee}$ by $a \sim a^{\prime}$ if and only if $\gamma(a)=\gamma\left(a^{\prime}\right)$. For any $k$-dimensional face $\gamma$ of $P$, there is an equivalence class $\gamma^{\vee}$ which is defined by

$$
\begin{aligned}
\gamma^{\vee}: & =\left\{a \in\left(\mathbb{R}^{n}\right)^{\vee}: \gamma(a)=\gamma \text { and } a_{j} \geq 0 \text { for } j=1, \ldots, n\right\} \\
( & \left.=\left\{a \in\left(\mathbb{R}^{n}\right)^{\vee}: \gamma=H(a, l(a)) \cap P \text { and } a_{j} \geq 0 \text { for } j=1, \ldots, n\right\} .\right)
\end{aligned}
$$

Here, $P^{\vee}:=\{0\}$. The closure of $\gamma^{\vee}$, denoted by $\overline{\gamma^{\vee}}$, is expressed as

$$
\overline{\gamma^{\vee}}=\left\{a \in\left(\mathbb{R}^{n}\right)^{\vee}: \gamma \subset H(a, l(a)) \cap P \text { and } a_{j} \geq 0 \text { for } f=1, \ldots, n\right\} .
$$

It is easy to see that $\overline{\gamma^{\vee}}$ is an $(n-k)$-dimensional strongly convex rational polyhedral cone in $\left(\mathbb{R}^{n}\right)^{\vee}$ and, moreover, that the collection of $\overline{\gamma^{\vee}}$ gives a fan $\Sigma_{P}$, which is called the fan associated with polyhedron $P$. Note that $\left|\Sigma_{P}\right|=\mathbb{R}_{+}^{n}$.

Furthermore, let us consider $n$-dimensional polyhedra $P_{1}, \ldots, P_{m} \subset \mathbb{R}_{+}^{n}$ satisfying $P_{j}+\mathbb{R}_{+}^{n} \subset P_{j}$ for all $j$. Let $\Sigma_{P_{j}}$ be the fan associated with $P_{j}$. It is easy to see that the collection of $\sigma_{1} \cap \cdots \cap \sigma_{m}$ for all $\sigma_{j} \in \Sigma_{P_{j}}$ gives a fan, which is called the fan associated with the polyhedra $P_{1}, \ldots, P_{m}$. We remark that any simplicial subdivision of this fan is also a simplicial subdivision of $\Sigma_{P_{j}}$ for each $j$.

6.2. Lemmas. Let us recall some lemmas given in [20]. They will be useful for the analysis in Sections 8 13. These lemmas also play important roles in the proofs of Lemma 7.1 and Theorem 7.2, which are concerned with toric resolutions of singularities in the class $\hat{\mathcal{E}}(U)$ (see [20]).

The following symbols are used in this subsection.

- $\Sigma_{0}$ is the fan associated with the polyhedron $P$;

- $\Sigma$ is a simplicial subdivision of $\Sigma_{0}$;

- $\Sigma^{(n)}$ consists of $n$-dimensional cones in $\Sigma$;

- $a^{1}(\sigma), \ldots, a^{n}(\sigma)$ is the skeleton of $\sigma \in \Sigma^{(n)}$, ordered once and for all;

- $\mathcal{P}(\{1, \ldots, n\})$ is the set of all subsets in $\{1, \ldots, n\}$;

- $\mathcal{F}[P]$ is the set of nonempty faces of $P$;

- $H(\cdot, \cdot), l(\cdot)$ are as in (2.1), (6.1), respectively. 
It is easy to see that the two maps

$$
\gamma: \mathcal{P}(\{1, \ldots, n\}) \times \Sigma^{(n)} \rightarrow \mathcal{F}[P] ; \quad I: \mathcal{F}[P] \times \Sigma^{(n)} \rightarrow \mathcal{P}(\{1, \ldots, n\})
$$

can be defined by

$$
\begin{aligned}
& \gamma(I, \sigma):=\bigcap_{j \in I} H\left(a^{j}(\sigma), l\left(a^{j}(\sigma)\right)\right) \cap P, \\
& I(\gamma, \sigma):=\left\{j: \gamma \subset H\left(a^{j}(\sigma), l\left(a^{j}(\sigma)\right)\right)\right\} .
\end{aligned}
$$

Here set $\gamma(\emptyset, \sigma):=P$. Note that $I(P, \sigma)=\emptyset$.

Lemma 6.1. For $\sigma \in \Sigma^{(n)}, \gamma \in \mathcal{F}[P], I \in \mathcal{P}(\{1, \ldots, n\})$, we have the following.

(i) $\gamma \subset \gamma(I(\gamma, \sigma), \sigma)$ and $\operatorname{dim}(\gamma) \leq n-\# I(\gamma, \sigma)$.

(ii) $\gamma=\gamma(I, \sigma) \Longrightarrow I \subset I(\gamma, \sigma) \Longrightarrow \operatorname{dim}(\gamma) \leq n-\# I$.

Proof. The assertions in (i) are directly seen from the definitions of $\gamma(I, \sigma)$ and $I(\gamma, \sigma)$. The first implication in (ii) is shown as follows: $\gamma=\gamma(I, \sigma) \Rightarrow \gamma=$ $\bigcap_{j \in I} H\left(a^{j}(\sigma), l\left(a^{j}(\sigma)\right)\right) \cap P \Rightarrow \gamma \subset H\left(a^{j}(\sigma), l\left(a^{j}(\sigma)\right)\right)$ for $j \in I \Rightarrow I \subset I(\gamma, \sigma)$. From the inequality in (i), the second implication in (ii) is obvious.

Next, consider the case when $\operatorname{dim}(\gamma)=n-\# I(\gamma, \sigma)$. Define

$$
\Sigma^{(n)}(\gamma):=\left\{\sigma \in \Sigma^{(n)}: \operatorname{dim}(\gamma)=n-\# I(\gamma, \sigma)\right\} .
$$

Note that $\Sigma^{(n)}(P)=\Sigma^{(n)}$.

Lemma 6.2. For $\sigma \in \Sigma^{(n)}, \gamma \in \mathcal{F}[P], I \in \mathcal{P}(\{1, \ldots, n\})$, we have the following. Here $\gamma^{\vee}$ is as in (6.2).

(i) $\# I(\gamma, \sigma)=\operatorname{dim}\left(\gamma^{\vee} \cap \sigma\right)$.

(ii) $\Sigma^{(n)}(\gamma)=\left\{\sigma \in \Sigma^{(n)}: \operatorname{dim}\left(\gamma^{\vee} \cap \sigma\right)=\operatorname{dim}\left(\gamma^{\vee}\right)\right\} \neq \emptyset$.

(iii) If $\sigma \in \Sigma^{(n)}(\gamma)$, then $\gamma=\gamma(I(\gamma, \sigma), \sigma)$.

Proof. (i) This equation follows from the equivalences

$$
j \in I(\gamma, \sigma) \Leftrightarrow \gamma \subset H\left(a^{j}(\sigma), l\left(a^{j}(\sigma)\right)\right) \Leftrightarrow a^{j}(\sigma) \in \overline{\gamma^{\vee}} \Leftrightarrow a^{j}(\sigma) \in \overline{\gamma^{\vee}} \cap \sigma,
$$

where $\overline{\gamma^{\vee}}$ denotes the closure of $\gamma^{\vee}$. Note that the second equivalence follows from (6.3).

(ii) Putting the equation in (i) and $\operatorname{dim}\left(\gamma^{\vee}\right)=n-\operatorname{dim}(\gamma)$ together, we see the equality of the sets. Since the support of the fan $\Sigma$ is $\mathbb{R}_{+}^{n}$, there exists $\sigma$ such that $\operatorname{dim}\left(\gamma^{\vee} \cap \sigma\right)=\operatorname{dim}\left(\gamma^{\vee}\right)$, which implies $\Sigma^{(n)}(\gamma) \neq \emptyset$.

(iii) Lemma 6.1 (i),(ii) and the assumption imply $\operatorname{dim}(\gamma)=\operatorname{dim}(\gamma(I(\gamma, \sigma), \sigma))$. In fact,

$$
\operatorname{dim}(\gamma) \leq \operatorname{dim}(\gamma(I(\gamma, \sigma), \sigma)) \leq n-\# I(\gamma, \sigma)=\operatorname{dim}(\gamma)
$$

Since $\gamma \subset \gamma(I(\gamma, \sigma), \sigma)$ from Lemma 6.1 (i), the above dimensional equation yields $\gamma=\gamma(I(\gamma, \sigma), \sigma)$.

Remark 6.3. It follows from Lemma 6.2 (ii) and (iii) that the map $\gamma$ is surjective.

The following lemma is concerned with the property of the map $\pi(\sigma)$ in (5.1) when the face $\gamma(I, \sigma)$ is compact.

Lemma 6.4. If $\gamma=\gamma(I, \sigma)$, then the following conditions are equivalent.

(i) $\gamma$ is compact;

(ii) $\pi(\sigma)\left(T_{I}\left(\mathbb{R}^{n}\right)\right)=0$.

Proof. See Proposition 8.6 in [20]. 


\section{Resolution of Singularities in the Class $\hat{\mathcal{E}}(U)$}

In this section, we recall a result in 20, about resolution of singularities in the class $\hat{\mathcal{E}}(U)$. Furthermore, we try to get a simultaneous resolution of singularities for several functions belonging to $\hat{\mathcal{E}}(U)$, which is crucial for our analysis.

Hereafter, the following symbols are often used:

$$
l_{f}(a):=\min \left\{\langle a, \alpha\rangle: \alpha \in \Gamma_{+}(f)\right\},
$$

where $f$ is a smooth function defined near the origin, and

$$
\begin{aligned}
& T_{I}\left(\mathbb{R}^{n}\right)=\left\{y \in \mathbb{R}^{n}: y_{j}=0 \text { if } j \in I\right\} \quad(\text { as in }(1.5)), \\
& \tilde{T}_{I}\left(\mathbb{R}^{n}\right):=\left\{y \in \mathbb{R}^{n}: y_{j}=0 \text { if and only if } j \in I\right\} \\
&\left(=\left\{y \in T_{I}\left(\mathbb{R}^{n}\right): y_{j} \neq 0 \text { if } j \notin I\right\}\right) .
\end{aligned}
$$

By using the lemmas in the previous section, we see that an $\hat{\mathcal{E}}$ function can be expressed as in the normal crossing form sufficiently near the origin. The proof is given by using the lemmas in the previous section. The details are seen in [20].

Lemma 7.1 ([20]). Let $f$ satisfy the condition (A) in Section 3 and belong to $\hat{\mathcal{E}}(U)$, where $U$ is a neighborhood of the origin, let $\Sigma$ be a simplicial subdivision of the fan $\Sigma_{0}$ associated with the Newton polyhedra $\Gamma_{+}(f)$ (see Section 6.1) and let $\sigma$ be an $n$-dimensional cone in $\Sigma$ whose skeleton is $a^{1}(\sigma), \ldots, a^{n}(\sigma) \in \mathbb{Z}_{+}^{n}$. Then for any $\sigma \in \Sigma^{(n)}$, there exists a smooth function $f_{\sigma}$ defined on the set $\pi(\sigma)^{-1}(U)$ such that $f_{\sigma}(0) \neq 0$ and

$$
f(\pi(\sigma)(y))=\left(\prod_{j=1}^{n} y_{j}^{l_{f}\left(a^{j}(\sigma)\right)}\right) f_{\sigma}(y) \quad \text { for } y \in \pi(\sigma)^{-1}(U) .
$$

Furthermore, if $\gamma=\gamma(I, \sigma)$ (see (6.4) $)$, then we have

$$
f_{\gamma}(\pi(\sigma)(y))=\left(\prod_{j=1}^{n} y_{j}^{l_{f}\left(a^{j}(\sigma)\right)}\right) f_{\sigma}\left(T_{I}(y)\right) \quad \text { for } y \in \pi(\sigma)^{-1}(U) .
$$

Notice that $f_{\sigma}$ may still have a singularity at a point where $f_{\sigma}$ vanishes. But an additional nondegeneracy assumption induces that these kinds of singularities do not appear. This fact is exactly stated as in Theorem 8.10 in the paper [20]. This result will be generalized in the following theorem. It shows the existence of some kind of simultaneous resolution of singularities for several functions belonging to the class $\hat{\mathcal{E}}(U)$ and satisfying the nondegeneracy condition.

Theorem 7.2. Let $f_{1}, \ldots, f_{m}$ satisfy the condition (A) in Section 3 and belong to $\hat{\mathcal{E}}(U)$, where $U$ is a neighborhood of the origin, let $\Sigma$ be a simplicial subdivision of the fan $\Sigma_{0}$ associated with the Newton polyhedra $\Gamma_{+}\left(f_{1}\right), \ldots, \Gamma_{+}\left(f_{m}\right)$ and let $\sigma$ be an $n$-dimensional cone in $\Sigma$, whose skeleton is $a^{1}(\sigma), \ldots, a^{n}(\sigma) \in \mathbb{Z}_{+}^{n}$. Then for any $k \in\{1, \ldots, m\}$ and any $\sigma \in \Sigma^{(n)}$, there exists a smooth function $f_{k, \sigma}$ defined on the set $\pi(\sigma)^{-1}(U)$ satisfying that $f_{k, \sigma}(0) \neq 0$ and

$$
f_{k}(\pi(\sigma)(y))=\left(\prod_{j=1}^{n} y_{j}^{l_{f_{k}}\left(a^{j}(\sigma)\right)}\right) f_{k, \sigma}(y)
$$

for $y \in \pi(\sigma)^{-1}(U)$, where $l_{f_{k}}(\cdot)$ is as in (7.1). 
Furthermore, if $f_{k}$ is nondegenerate over $\mathbb{R}$ with respect to $\Gamma_{+}\left(f_{k}\right)$ and a set $I \subset\{1, \ldots, n\}$ satisfies $\pi(\sigma)\left(\tilde{T}_{I}\left(\mathbb{R}^{n}\right)\right)=0$, then the set $\left\{y \in \tilde{T}_{I}\left(\mathbb{R}^{n}\right): f_{k, \sigma}(y)=0\right\}$ is nonsingular; i.e., the gradient of the restriction of the function $f_{k, \sigma}$ to $\tilde{T}_{I}\left(\mathbb{R}^{n}\right)$ does not vanish at the points of the set $\left\{y \in \tilde{T}_{I}\left(\mathbb{R}^{n}\right): f_{k, \sigma}(y)=0\right\}$.

Remark 7.3. Let $b=\left(b_{1}, \ldots, b_{n}\right)$ be a point on $\tilde{T}_{I}\left(\mathbb{R}^{n}\right)$ satisfying $f_{k, \sigma}(b)=0$. By the implicit function theorem, there exists a local coordinate around $b$ in which $f_{k} \circ \pi(\sigma)$ can be expressed in a normal crossing form. To be more specific, there exists a local diffeomorphism $\phi$ defined around $b$ such that $y=\phi(u)$ with $b=\phi(b)$ and

$$
\left(f_{k} \circ \pi(\sigma) \circ \phi\right)(u)=\left(u_{i}-b_{i}\right)\left(\prod_{j \in I} u_{j}^{l_{f_{k}}\left(a^{j}(\sigma)\right)}\right),
$$

where $y_{j}=u_{j}$ for $j \in I$ and $i \in\{1, \ldots, n\} \backslash I$.

Proof of Theorem 7.2. The case $m=1$ in the theorem has been shown in 20. The case $m \geq 2$ can be similarly shown by using the fact that $\Sigma$ is a simplicial subdivision of each fan $\Sigma_{f_{k}}$ for all $k$, where $\Sigma_{f_{k}}$ is the fan associated with $\Gamma_{+}\left(f_{k}\right)$.

7.1. Remarks. The proposition, below, is a weaker version of Theorem 7.2. But, when one wants to get a simultaneous resolution of singularities with respect to several functions, it might be more convenient to deal with their product. Indeed, after computing the product, we can construct the resolution via only one function from this proposition.

Proposition 7.4. Let $f_{1}, \ldots, f_{m}$ be as in Theorem 7.2 and let $F(x):=\prod_{k=1}^{m} f_{k}(x)$. Let $\Sigma$ be a simplicial subdivision of the fan $\Sigma_{F}$ associated with the Newton polyhedron $\Gamma_{+}(F)$ and let $\sigma$ be an $n$-dimensional cone in $\Sigma$ whose skeleton is $a^{1}(\sigma), \ldots$, $a^{n}(\sigma) \in \mathbb{Z}_{+}^{n}$. Then for any $k \in\{1, \ldots, m\}$ and any $\sigma \in \Sigma^{(n)}$, there exists a smooth function $f_{k, \sigma}$ defined on the set $\pi(\sigma)^{-1}(U)$ satisfying that $f_{k, \sigma}(0) \neq 0$ and the equation (7.5) holds for $y \in \pi(\sigma)^{-1}(U)$.

Furthermore, if $F$ is nondegenerate over $\mathbb{R}$ with respect to $\Gamma_{+}(F)$ and a set $I \subset\{1, \ldots, n\}$ satisfies $\pi(\sigma)\left(\tilde{T}_{I}\left(\mathbb{R}^{n}\right)\right)=0$, then the set $\left\{y \in \tilde{T}_{I}\left(\mathbb{R}^{n}\right): f_{k, \sigma}(y)=0\right\}$ is nonsingular.

In order to prove Proposition 7.4, the following lemmas are important. We denote $\mathbb{R}_{>0}^{n}:=\left\{x=\left(x_{1}, \ldots, x_{n}\right) \in \mathbb{R}^{n}: x_{j}>0\right.$ for $\left.j=1, \ldots, n\right\}$.

Lemma 7.5. Let $a=\left(a_{1} \ldots, a_{n}\right) \in \mathbb{R}_{>0}^{n}$. Let $\Gamma(a):=H\left(a, l_{F}(a)\right) \cap \Gamma_{+}(F)$ and $\gamma_{k}(a):=H\left(a, l_{f_{k}}(a)\right) \cap \Gamma_{+}\left(f_{k}\right)$ for $k=1, \ldots, m$. (Note that the faces $\Gamma(a)$ and $\gamma_{k}(a)$ are compact for $k=1, \ldots, m$.) Then we have

(i) $F_{\Gamma(a)}(x)=\prod_{k=1}^{m}\left(f_{k}\right)_{\gamma_{k}(a)}(x)$;

(ii) $l_{F}(a)=\sum_{k=1}^{m} l_{f_{k}}(a)$,

where $F_{\Gamma(a)}$ is the $\Gamma(a)$-part of $F$ and $\left(f_{k}\right)_{\gamma_{k}(a)}$ is the $\gamma_{k}(a)$-part of $f_{k}$ for $k=$ $1, \ldots, n$. Furthermore, if every $f_{k}$ belongs to $\hat{\mathcal{E}}(U)$ for $k=1, \ldots, m$, then the above (i), (ii) hold for any $a \in \mathbb{R}_{+}^{n}$.

Proof. The following equation holds:

$$
\frac{F\left(t^{a_{1}} x_{1}, \ldots, t^{a_{n}} x_{n}\right)}{t^{l_{F}(a)}}=\left(\prod_{k=1}^{m} \frac{f_{k}\left(t^{a_{1}} x_{1}, \ldots, t^{a_{n}} x_{n}\right)}{t^{l_{f_{k}}(a)}}\right) \cdot t^{\sum_{k=1}^{m} l_{f_{k}}(a)-l_{F}(a)},
$$


where $a \in \mathbb{R}_{+}^{n}, t>0$ is small and $x$ is in a small neighborhood of the origin. Consider the limits as $t \rightarrow 0$ of the left and right sides of the above equation. Then the lemma can be easily shown.

Lemma 7.6. Let $a=\left(a_{1} \ldots, a_{n}\right) \in \mathbb{R}_{>0}^{n}$. Let $f$ be a smooth function satisfying the condition (A) in Section $3, \Sigma_{f}$ the fan associated to $\Gamma_{+}(f)$ and $\gamma(a)$ the compact face of $\Gamma_{+}(f)$ defined by $H\left(a, l_{f}(a)\right) \cap \Gamma_{+}(f)$. Then the following two conditions are equivalent.

(i) The $\gamma(a)$-part $f_{\gamma(a)}$ of $f$ equals a monomial.

(ii) There exists an $n$-dimensional cone in $\Sigma_{f}$ such that $a \in \operatorname{Int}(\sigma)$, where $\operatorname{Int}(\sigma)$ means the interior of $\sigma$.

Proof. Since $a \in \mathbb{R}_{>0}^{n}, \gamma(a)$ is a vertex if and only if $f_{\gamma(a)}$ is a monomial. Moreover, from the definition of cone in Section 6.1, the face $\gamma(a)$ is a vertex if and only if $a$ belongs to the interior of some $n$-dimensional cone of $\Sigma_{f}$.

Lemma 7.7. Let $\Sigma_{0}$ be the fan associated with the Newton polyhedra $\Gamma_{+}\left(f_{1}\right), \ldots$, $\Gamma_{+}\left(f_{m}\right)$ (as in Theorem 7.2). Then we have $\Sigma_{F}=\Sigma_{0}$.

Proof. By an inductive argument, it suffices to show the lemma in the case when $m=2$. Let $\Sigma_{F}^{(n)}$ (resp. $\Sigma_{f_{1}}^{(n)}, \Sigma_{f_{2}}^{(n)}, \Sigma_{0}^{(n)}$ ) be the set of $n$-dimensional cones contained in $\Sigma_{F}$ (resp. $\left.\Sigma_{f_{1}}, \Sigma_{f_{2}}, \Sigma_{0}\right)$.

Let us consider the following two claims:

(a) For any $\sigma \in \Sigma_{F}^{(n)}$, there exist $\sigma_{1} \in \Sigma_{f_{1}}^{(n)}$ and $\sigma_{2} \in \Sigma_{f_{2}}^{(n)}$ such that $\sigma \subset \sigma_{1} \cap \sigma_{2}$.

(b) For any $\sigma_{1} \in \Sigma_{f_{1}}^{(n)}$ and $\sigma_{2} \in \Sigma_{f_{2}}^{(n)}$, there exists $\sigma \in \Sigma_{F}^{(n)}$ such that $\operatorname{Int}\left(\sigma_{1} \cap \sigma_{2}\right) \subset \sigma$.

[Proof of the claim (a).] Let $\sigma \in \Sigma_{F}^{(n)}$. Since $F_{\Gamma(a)}(x)$ is a monomial for any $a \in$ $\operatorname{Int}(\sigma)$ from Lemma 7.6 and $F_{\Gamma(a)}(x)=\left(f_{1}\right)_{\gamma_{1}(a)}(x) \cdot\left(f_{2}\right)_{\gamma_{2}(a)}(x)$ from Lemma 7.5 (i), $\left(f_{1}\right)_{\gamma_{1}(a)}(x)$ and $\left(f_{2}\right)_{\gamma_{2}(a)}(x)$ are monomials. Applying Lemma 7.5 again, we see that $a \in \operatorname{Int}\left(\sigma_{1}\right) \cap \operatorname{Int}\left(\sigma_{2}\right)=\operatorname{Int}\left(\sigma_{1} \cap \sigma_{2}\right)$.

[Proof of the claim (b).] We only consider the case when $\operatorname{Int}\left(\sigma_{1} \cap \sigma_{2}\right) \neq \emptyset$. If $a \in \operatorname{Int}\left(\sigma_{1} \cap \sigma_{2}\right)$, then Lemmas 7.5 and 7.6 imply that $F_{\Gamma(a)}(x)$ is a monomial. Thus there exists a cone $\sigma \in \Sigma_{F}^{(n)}$ such that $a \in \operatorname{Int}(\sigma)$.

By using the above two claims (a), (b), the lemma can be shown as follows. For any $\sigma \in \Sigma_{F}^{(n)}$, there exist $\sigma_{1} \in \Sigma_{f_{1}}^{(n)}, \sigma_{2} \in \Sigma_{f_{2}}^{(n)}$ and $\tilde{\sigma} \in \Sigma_{F}^{(n)}$ such that $\sigma \subset \sigma_{1} \cap \sigma_{2} \subset \tilde{\sigma}$. Since $\sigma$ and $\tilde{\sigma}$ belong to $\Sigma_{F}^{(n)}$ and satisfy $\sigma \subset \tilde{\sigma}, \sigma$ and $\tilde{\sigma}$ are the same cones. Thus $\sigma=\sigma_{1} \cap \sigma_{2} \in \Sigma_{0}^{(n)}$, which implies $\Sigma_{F}^{(n)} \subset \Sigma_{0}^{(n)}$. On the other hand, the inclusion $\Sigma_{0}^{(n)} \subset \Sigma_{F}^{(n)}$ can be similarly shown. Therefore, we see that the two fans $\Sigma_{0}$ and $\Sigma_{F}$ are the same.

Lemma 7.8. If $F$ is nondegenerate over $\mathbb{R}$ with respect to its Newton polyhedron, then so is every $f_{k}$ for $k=1, \ldots, m$.

Proof. By an inductive argument, it also suffices to show the lemma in the case when $m=2$.

Suppose that $f_{1}$ is not nondegenerate. Then there exists a compact face $\gamma$ of $\Gamma_{+}\left(f_{1}\right)$ and a point $x_{*} \in(\mathbb{R} \backslash\{0\})^{n}$ such that $\frac{\partial\left(f_{1}\right)_{\gamma}}{\partial x_{j}}\left(x_{*}\right)=0$ for $j=1, \ldots, n$. Let $a \in \mathbb{R}_{>0}^{n}$ satisfy that $\left(a, l_{f_{1}}(a)\right)$ is a valid pair defining $\gamma$. Moreover, from 
the Euler identity, $\sum_{j=1}^{n} a_{j} x_{j} \frac{\partial\left(f_{1}\right)_{\gamma}}{\partial x_{j}}(x)=\left(f_{1}\right)_{\gamma}(x)$, we have $\left(f_{1}\right)_{\gamma}\left(x_{*}\right)=0$. Since $F_{\Gamma(a)}(x)=\left(f_{1}\right)_{\gamma}(x) \cdot\left(f_{2}\right)_{\gamma_{2}(a)}(x)$ from Lemma $7.5(\mathrm{i})$, we have

$$
\frac{\partial F_{\Gamma(a)}}{\partial x_{j}}\left(x_{*}\right)=\frac{\partial\left(f_{1}\right)_{\gamma}}{\partial x_{j}}\left(x_{*}\right) \cdot\left(f_{2}\right)_{\gamma_{2}(a)}\left(x_{*}\right)+\left(f_{1}\right)_{\gamma}\left(x_{*}\right) \cdot \frac{\partial\left(f_{2}\right)_{\gamma_{2}(a)}}{\partial x_{j}}\left(x_{*}\right)=0,
$$

for $j=1, \ldots, m$. This shows that $F$ is not nondegenerate.

Remark 7.9. The converse of the assertion in Lemma 7.8 does not hold in general. Indeed, consider the case $f_{1}\left(x_{1}, x_{2}\right)=f_{2}\left(x_{1}, x_{2}\right)=x_{1}^{2}-x_{2}^{2}$. Therefore, the assumptions in Proposition 7.4 are stronger than those in Theorem 7.2.

Proof of Proposition 7.4. Applying Lemmas 7.7 and 7.8 to Theorem 7.2, we can easily show the proposition.

\section{Poles of local Zeta type FUnCtions}

The subsequent six sections are devoted to the investigation of poles of weighted local zeta functions and similar functions. Throughout these sections, we usually assume the following without any mentioning: Let $U$ be an open neighborhood of the origin in $\mathbb{R}^{n}$ and the functions $f, g, \varphi$ satisfy the conditions (A), (B), (C) in the beginning of Section 3. But we sometimes consider the case when $g$ is a nonzero flat function in Section 10, which does not satisfy the condition (B).

By means of the Mellin transform, there exists a clear relationship between the oscillatory integral (1.1) and some functions similar to the local zeta function (1.3) (see Section 14.1). As is well known in [19, [22, [30, [18, [1, etc., through this relationship, the investigation of the behavior of oscillatory integrals is reduced to that of the properties of poles of these functions. The results in Section 4 will be proved in Section 14.3 by using the results in these six sections.

8.1. Functions similar to the local zeta functions. We investigate the properties of poles of the functions

$$
Z_{+}(s ; \varphi)=\int_{\mathbb{R}^{n}} f(x)_{+}^{s} g(x) \varphi(x) d x, \quad Z_{-}(s ; \varphi)=\int_{\mathbb{R}^{n}} f(x)_{-}^{s} g(x) \varphi(x) d x,
$$

where

$$
f(x)_{+}=\max \{f(x), 0\} \text { and } f(x)_{-}=\max \{-f(x), 0\},
$$

and the (weighted) local zeta function

$$
Z(s ; \varphi)=\int_{\mathbb{R}^{n}}|f(x)|^{s} g(x) \varphi(x) d x .
$$

We call $g$ the weight. From the properties of $Z_{+}(s ; \varphi)$ and $Z_{-}(s ; \varphi)$, we can easily obtain analogous properties of $Z(s ; \varphi)$ by using the relationship $Z(s ; \varphi)=$ $Z_{+}(s ; \varphi)+Z_{-}(s ; \varphi)$.

It is easy to see that $Z_{ \pm}(s ; \varphi)$ and $Z(s ; \varphi)$ are holomorphic functions on the region $\operatorname{Re}(s)>0$. Moreover, if there exists a "simultaneous resolution of singularities" with respect to the functions $f$ and $g$, then $Z_{ \pm}(s ; \varphi)$ and $Z(s ; \varphi)$ can be meromorphically continued to the whole complex plane and, moreover, precise properties of their poles can be seen. Following the analysis of Varchenko in [30] (see also [1]), let us explain this process. 
8.2. How to use resolution of singularities. For the moment, we assume the existence of a proper real analytic mapping $\pi$ from some $n$-dimensional real analytic manifold $Y$ to $\mathbb{R}^{n}$ such that at each point of the set $\pi^{-1}(0)$, there exist smooth local coordinates $y=\left(y_{1}, \ldots, y_{n}\right)$ satisfying the following properties:

(i) There exist nonnegative integers $l_{j}, \tilde{l}_{j}$ and smooth functions $\tilde{f}, \tilde{g}$ defined near the origin with $\tilde{f}(0), \tilde{g}(0) \neq 0$ such that

$$
f(\pi(y))=\left(\prod_{j=1}^{n} y_{j}^{l_{j}}\right) \tilde{f}(y), \quad g(\pi(y))=\left(\prod_{j=1}^{n} y_{j}^{\tilde{l}_{j}}\right) \tilde{g}(y) .
$$

(ii) The Jacobian of the mapping $\pi$ has the form

$$
J_{\pi}(y)= \pm \prod_{j=1}^{n} y_{j}^{\tilde{m}_{j}-1}
$$

where $\tilde{m}_{j}$ are positive integers.

(iii) On a neighborhood of the origin in $\mathbb{R}^{n}, \pi$ is a diffeomorphism outside a proper analytic subset in $\mathbb{R}^{n}$.

The above proper map $\pi: Y \rightarrow \mathbb{R}^{n}$ is called a simultaneous resolution of singularities with respect to $f$ and $g$.

By means of the above map $\pi: Y \rightarrow \mathbb{R}^{n}$, we have

$$
\begin{aligned}
Z_{ \pm}(s ; \varphi)=\int_{\mathbb{R}^{n}} f(x)_{ \pm}^{s} g(x) \varphi(x) d x \\
\quad=\int_{Y} f(\pi(y))_{ \pm}^{s} g(\pi(y)) \varphi(\pi(y)) J_{\pi}(y) d y .
\end{aligned}
$$

Furthermore, by using an appropriate smooth finite partition of unity, substituting (8.3), (8.4) and applying the orthant decomposition

$$
\int_{\mathbb{R}^{n}} F(x) d x=\sum_{\theta \in\{-1,1\}^{n}} \int_{\mathbb{R}_{+}^{n}} F\left(\theta_{1} x_{1}, \ldots, \theta_{n} x_{n}\right) d x,
$$

where $F$ is a continuous function on $\mathbb{R}^{n}$, (8.5) implies that the functions $Z_{ \pm}(s ; \varphi)$ can be written in a certain sum of functions of the following form:

$$
\int_{\mathbb{R}_{+}^{n}}\left(\prod_{j=1}^{n} y_{j}^{l_{j} s+\tilde{l}_{j}+\tilde{m}_{j}-1}\right) \psi(y ; s) d y,
$$

where $\psi(\cdot ; s)$ is a $C_{0}^{\infty}$ function of $y$ on $\mathbb{R}^{n}$ for any $s \in \mathbb{C}$ and $\psi(y ; \cdot)$ is an entire function on $\mathbb{C}$ for any $y \in \mathbb{R}^{n}$. As we will show in the next section, the above integral is easily treated, and a direct computation gives many important properties of its poles. From the above arguments, it becomes an essentially important problem to find an appropriate map $\pi$ in our investigation of the functions $Z_{ \pm}(s ; \varphi)$ and the oscillatory integral $I(t ; \varphi)$.

Let us discuss the existence of the simultaneous resolution of singularities with respect to $f$ and $g$ and its application in earlier studies of oscillatory integrals. 
First, we consider the unweighted case (i.e., $g \equiv 1$ ). If $f$ is real analytic near the origin, then Hironaka's famous theorem [13] implies the existence of a desired map $\pi: Y \rightarrow \mathbb{R}^{n}$ (see also [2]). Moreover, an easy computation in the next section shows that poles of the integral (8.7) are contained in arithmetic progressions. As a result, one can see that the distribution of poles of $Z_{ \pm}(s ; \varphi)$ are contained in a union of a finite number of arithmetic progressions and, moreover, that an asymptotic expansion of the oscillatory integral $I(t ; \varphi)$ in (1.1) can be expressed as in (1.2). These analyses are seen in the earlier works [19], 22], etc. This kind of application of Hironaka's resolution theorem to the analysis can be traced back to the works of Berenstein and Gel'fand [3] and Atiyah [2. By the way, these results are still very abstract. In order to determine or exactly estimate the oscillation index and its multiplicity, one has to get more quantitative information of the leading pole of the integrals in (8.1). Varchenko [30] constructs resolution of singularities by using the theory of toric varieties and describes the values of $l_{j}, \tilde{m}_{j}$ in (8.3), (8.4) in terms of the geometrical information of the Newton polyhedron of $f$ under the assumption (D) in Section 3. As a result, he gave precise results in Theorem 3.1 in Section 3. Furthermore, the authors 20] obtain an analogous result when $f$ satisfies the condition:

(E) $f$ belongs to the class $\hat{\mathcal{E}}(U)$ and is nondegenerate over $\mathbb{R}$ with respect to its Newton polyhedron.

Next, let us consider the weighted case (i.e., $g \not \equiv 1$ ). When the weight is a monomial, the above analysis of Varchenko can be directly generalized, and the values of $\tilde{l}_{j}$ are also computed by using the resolution for the function $f$ only (see 31] and Section 10 in this paper). A more general weighted case essentially requires a simultaneous resolution of singularities with respect to $f$ and $g$. Remember that a map $\pi: Y_{\Sigma} \rightarrow \mathbb{R}^{n}$ in Section 7 has been constructed in the case of $\hat{\mathcal{E}}(U)$, which is similar to the simultaneous resolution of singularities with respect to $f$ and $g$, by using the geometrical information of the Newton polyhedra of $f$ and $g$ under some conditions. Though this map $\pi$ does not satisfy all the conditions for resolution of singularities with respect to $f$ and $g$, we can obtain sufficiently precise results about the poles of $Z_{ \pm}(s ; \varphi)$ by means of this map in Sections 10, 11 and 12.

\section{Computation on poles of the elementary integrals}

In this section, we investigate poles of an elementary integral of the form

$$
L(s)=\int_{\mathbb{R}_{+}^{n}}\left(\prod_{j=1}^{n} y_{j}^{l_{j} s+m_{j}-1}\right) \psi(y ; s) d y,
$$

where $l_{j} \in \mathbb{Z}_{+}, m_{j} \in \mathbb{N}$ and $\psi$ is as in (8.7). (Put $m_{j}=\tilde{l}_{j}+\tilde{m}_{j}$; then (8.7) is given.)

9.1. One-dimensional model. By repeated integrals, the analysis of poles of the integral $L(s)$ is essentially reduced to that of the following simple one-dimensional integral:

$$
L_{1}(s)=\int_{0}^{\infty} y^{s} \psi(y) d y
$$

where $\psi \in C_{0}^{\infty}(\mathbb{R})$. Observing the convergence of the integral, we see that $L_{1}$ is holomorphic on the region $\operatorname{Re}(s)>-1$. Furthermore, we obtain the following. 
Lemma 9.1. The function $L_{1}$ can be meromorphically extended to the whole complex plane and its poles are contained in $(-\mathbb{N})$. Moreover, we have

$$
\lim _{s \rightarrow-k}(s+k) L_{1}(s)=\frac{1}{(k-1) !} \psi^{(k-1)}(0) \quad \text { for } k \in \mathbb{N} .
$$

In particular,

$$
\lim _{s \rightarrow-1}(s+1) L_{1}(s)=\psi(0) .
$$

Proof. The integrations by parts imply

$$
\begin{aligned}
\int_{0}^{\infty} y^{s} \psi(y) d y & =\frac{-1}{s+1} \int_{0}^{\infty} y^{s+1} \psi^{\prime}(y) d y \\
=\cdots & =\frac{(-1)^{k}}{(s+1) \cdots(s+k)} \int_{0}^{\infty} y^{s+k} \psi^{(k)}(y) d y
\end{aligned}
$$

for any nonnegative integer $k$. The assertions in the lemma easily follow from the above equations.

9.2. Positions and orders of poles. Observing the one-dimensional model, we can see important properties of poles of the function $L(s)$ in (9.1). Let

$$
B:=\left\{j: l_{j} \neq 0\right\} \subset\{1, \ldots, n\} .
$$

It is easy to see that $L(s)$ is holomorphic on the region $\operatorname{Re}(s)>\max \left\{-\left(m_{j}-1\right) / l_{j}\right.$ : $j \in B\}$. Moreover, the following can be seen.

Proposition $9.2(9]$, 1]). The function $L(s)$ can be analytically continued to the whole complex plane as a meromorphic function and its poles belong to the set

$$
\left\{-\frac{m_{j}+\nu_{j}}{l_{j}}: \nu_{j} \in \mathbb{Z}_{+}, j \in B\right\} \text {. }
$$

Moreover, suppose that $p$ belongs to the above set and let

$$
A(p)=\left\{j \in B: \text { there exists } \nu_{j} \in \mathbb{Z}_{+} \text {such that }-\frac{m_{j}+\nu_{j}}{l_{j}}=p\right\} .
$$

Then, if $p$ is a pole of $L(s)$, then the order of the pole of $L(s)$ at $p$ is at most \# $A(p)$.

Proof. Observing the proof of Lemma 9.1, we can easily show the above from the integrations by parts with respect to $y_{j}$ for all $j \in B$.

Here, we return to the investigation of poles of $Z_{ \pm}(s ; \varphi), Z(s ; \varphi)$ in Section 8 , When there exists a "simultaneous resolution of singularities" with respect to $f$ and $g, Z(s ; \varphi), Z_{ \pm}(s ; \varphi)$ can be written in a certain sum of the integrals of the form (9.1). Applying Proposition 9.2 to these integrals, we see that their poles are contained in some union of sets of the form (9.6).

9.3. Coefficients. Let us compute the coefficients of terms in the Laurent expansion of $L(s)$ at the above candidate poles. This computation will be useful for the investigations in the latter sections.

Let $s_{*}:=\max \left\{-m_{j} / l_{j}: j \in B\right\}$ and $A_{*}:=A\left(s_{*}\right) \subset B$. Note that $s_{*}$ is the leading pole of $L(s)$ and \# $A_{*}$ is its order if the following coefficient does not vanish. 
Proposition 9.3. The coefficient of $\left(s-s_{*}\right)^{-\# A_{*}}$ in the Laurent expansion of $L(s)$ at $s=s_{*}$ is equal to

$$
\left\{\begin{array}{l}
\frac{\psi\left(0 ; s_{*}\right)}{\prod_{j=1}^{n} l_{j}} \quad \text { if } A_{*}=B=\{1, \ldots, n\}, \\
\frac{1}{\prod_{j \in A_{*}} l_{j}} \int_{\mathbb{R}_{+}^{n-\# A_{*}}}\left(\prod_{j \notin A_{*}} y_{j}^{l_{j} s_{*}+m_{j}-1}\right) \psi\left(T_{A_{*}}(y) ; s_{*}\right) \prod_{j \notin A_{*}} d y_{j} \text { otherwise, }
\end{array}\right.
$$

where $T_{A_{*}}(\cdot)$ is as in (1.4).

Proof. Recalling the proof of Lemma 9.1, we see that the integration by parts with respect to each $y_{j}$ for $j \in A_{*}$ and the computation of $\lim _{s \rightarrow s_{*}}\left(s-s_{*}\right) \# A_{*} L(s)$ give the lemma.

In the analysis of $Z_{ \pm}(s ; \varphi)$ in the latter sections, we must consider the case when the coefficients of some poles have particular properties. (Actually, this case is induced by the zero set of the function $f_{\sigma}$ in Lemma [7.1.) This property will be understood through the following functions:

$$
L_{ \pm}(s)=\int_{\mathbb{R}_{+}^{n}}\left(y_{n}^{s} \prod_{j \in D} y_{j}^{l_{j} s+m_{j}-1}\right) \psi\left(y_{1}, \ldots, y_{n-1}, \pm y_{n}\right) d y
$$

where $D$ is a subset of $B \backslash\{n\}$.

Lemma 9.4. For $\lambda \in-\mathbb{N}$, let $A_{\lambda}$ be a subset in $D$ defined by $A_{\lambda}=\left\{j: l_{j} \lambda+m_{j}-\right.$ $1 \in-\mathbb{N}\}$. Then the functions $L_{ \pm}(s)$ have at $s=\lambda$ poles of order not higher than $\# A_{\lambda}+1$. Moreover, the following holds: Let $a_{\lambda}^{ \pm}$be the coefficients of $(s-\lambda)^{-\# A_{\lambda}-1}$ in the Laurent expansions of $L_{ \pm}(s)$ at $s=\lambda$, respectively. Then the equation $a_{\lambda}^{+}=(-1)^{\lambda-1} a_{\lambda}^{-}$for $\lambda \in-\mathbb{N}$ holds.

Proof. Let $k_{j}$ be positive integers defined by $k_{j}=\min \left\{k \in \mathbb{N}: l_{j} \lambda+m_{j}+k>0\right\}$ for $j \in D$. We remark that $l_{j} \lambda+m_{j}+k_{j}=1$ if and only if $j \in A_{\lambda}$. It easily follows from Proposition 9.2 that the functions $L_{ \pm}(s)$ can be analytically extended to $\mathbb{C}$ as meromorphic functions and they have at $s=\lambda$ poles of order not higher than $\# A_{\lambda}+1$.

By using the integration by parts, a direct computation gives $a_{\lambda}^{ \pm}=( \pm 1)^{\lambda-1} C_{\lambda}$, where $C_{\lambda}$ is given as follows. If $D=A_{\lambda}=\{1, \ldots, n-1\}$, then

$$
C_{\lambda}=\frac{1}{(-\lambda-1) !}\left(\prod_{j=1}^{n-1} \frac{1}{l_{j}\left(-l_{j} \lambda-m_{j}\right) !}\right)\left(\partial^{\alpha} \psi\right)(0) .
$$

Otherwise,

$$
\begin{aligned}
C_{\lambda}= & \frac{1}{(-\lambda-1) !}\left(\prod_{j \in A_{\lambda}} \frac{1}{l_{j}\left(-l_{j} \lambda-m_{j}\right) !}\right)\left(\prod_{j \in D \backslash A_{\lambda}} \frac{1}{\prod_{\nu=1}^{k_{j}}\left(-l_{j} \lambda-m_{j}+1-\nu\right)}\right) \\
& \times \int_{\mathbb{R}_{+}^{n-\# A_{\lambda}-1}}\left(\prod_{j \in D \backslash A_{\lambda}} y_{j}^{l_{j} \lambda+m_{j}+k_{j}-1}\right)\left(\partial^{\alpha} \psi\right)\left(T_{A_{\lambda} \cup\{n\}}(y)\right) \prod_{j \notin A_{\lambda} \cup\{n\}} d y_{j} .
\end{aligned}
$$

Here $\alpha=\left(\alpha_{1}, \ldots, \alpha_{n}\right)$ satisfies that $\alpha_{j}=k_{j}-1$ for $j \in A_{\lambda}, \alpha_{j}=k_{j}$ for $j \in D \backslash A_{\lambda}$, $\alpha_{n}=-\lambda-1$ and $\alpha_{j}=0$ otherwise. From the above equations, we see that $a_{\lambda}^{+}=(-1)^{\lambda-1} a_{\lambda}^{-}$. 


\section{The CASE OF MONOMIAL TYPE WEIGHT}

The purpose of this section is to investigate the poles of $Z_{ \pm}(s ; \varphi)$ in (8.1) and $Z(s ; \varphi)$ in (8.2) in the case when the weight $g$ is expressed as a monomial $x^{p}$ multiplied by a smooth function $\psi$ defined on a neighborhood $U$ of the origin in $\mathbb{R}^{n}$. In this case, $g$ does not belong to $\hat{\mathcal{E}}(U)$ if and only if $\psi$ is a nonzero flat function.

We explain reasons why we treat this special weight case in detail. First of all, this weight has a special form such that the analysis of Varchenko can be directly generalized. Moreover, many parts of the investigation of a more general weight case in Section 11 coincide with those of this simpler case. Therefore, the analysis in this section not only reduces the complexity of that in a more general weight case in Section 11 but also clarifies essentially important parts. Second of all, in this case, some particular results can be obtained, which cannot be covered by the results in Section 11. To be more specific, we consider the case when the weight is flat in this section (see Remark 10.3. below). This result affects the phenomenon in the behavior of oscillatory integrals in Remark 4.3 .

In this section, we use the following notation, introduced in Sections 5 and 6 :

- $\Sigma_{0}$ is the fan associated with $\Gamma_{+}(f)$;

- $\Sigma$ is a simplicial subdivision of $\Sigma_{0}$;

- $\left(Y_{\Sigma}, \pi\right)$ is the real resolution associated with $\Sigma$;

- $a^{1}(\sigma), \ldots, a^{n}(\sigma)$ is the skeleton of $\sigma \in \Sigma^{(n)}$, ordered once and for all;

- $J_{\pi}(y)$ is the Jacobian of the mapping of $\pi$.

10.1. Candidate poles. First, let us state our results relating to the positions and the orders of candidate poles of $Z_{ \pm}(s ; \varphi)$ and $Z(s ; \varphi)$.

Theorem 10.1. Suppose that (i) $f$ satisfies the condition (E), (ii) $g(x)=x^{p} \psi(x)$, where $p \in \mathbb{Z}_{+}^{n}$ and $\psi$ is a smooth function defined on $U$. If the support of $\varphi$ is contained in a sufficiently small neighborhood of the origin, then the functions $Z_{ \pm}(s ; \varphi)$ and $Z(s ; \varphi)$ can be analytically continued as meromorphic functions on the whole complex plane. More precisely, we have the following.

(a) The poles of the functions $Z_{ \pm}(s ; \varphi)$ and $Z(s ; \varphi)$ are contained in the set

$$
\left\{-\frac{\langle a, p+\mathbf{1}\rangle+\nu}{l_{f}(a)}: \nu \in \mathbb{Z}_{+}, a \in \tilde{\Sigma}^{(1)}\right\} \cup(-\mathbb{N}),
$$

where $l_{f}(a)$ is as in (7.1) and $\tilde{\Sigma}^{(1)}=\left\{a \in \Sigma^{(1)}: l_{f}(a) \neq 0\right\}$.

(b) The largest element of the first set in (10.1) is $-1 / d\left(f, x^{p}\right)$.

(c) When $Z_{ \pm}(s ; \varphi)$ and $Z(s ; \varphi)$ have poles at $s=-1 / d\left(f, x^{p}\right)$, their orders are at most

$$
\begin{cases}m\left(f, x^{p}\right) & \text { if } 1 / d\left(f, x^{p}\right) \text { is not an integer, } \\ \min \left\{m\left(f, x^{p}\right)+1, n\right\} & \text { otherwise. }\end{cases}
$$

Remark 10.2. When $\psi(0) \neq 0, \Gamma_{+}(g)=\{p\}+\mathbb{R}_{+}^{n}$, which implies $d\left(f, x^{p}\right)=d(f, g)$. Note that the equation $d\left(f, x^{p}\right)=d(f, g)$ does not always hold when $\psi(0)=0$.

Remark 10.3. When $\psi$ vanishes in high order at the origin, it is natural to expect that the set of the actual poles might become smaller than (10.1); in other words, the actual largest element in (b) might become smaller than $-1 / d\left(f, x^{p}\right)$. But, there exists a simple example of $f$ and $g$ violating this expectation (see Section 15.1). 
Indeed, this example shows that the set (10.1) is necessary to express the set of poles. Furthermore, we can see that the assertions in the above theorem hold even in the case when the weight $g$ is flat (equivalently $\psi$ is flat) and that they are optimal in the above sense.

Proof. We only show the assertions in the theorem in the case of the functions $Z_{ \pm}(s ; \varphi)$.

Step 1 (Orthant decompositions of $Z_{ \pm}(s ; \varphi)$ ). Applying the orthant decomposition (8.6) to the functions $Z_{ \pm}(s ; \varphi)$, we have

$$
Z_{ \pm}(s ; \varphi)=\sum_{\theta \in\{-1,1\}^{n}} \tilde{Z}_{ \pm}\left(s ; \varphi_{\theta} ; f_{\theta}, g_{\theta}\right),
$$

where $\varphi_{\theta}(x):=\varphi\left(\theta_{1} x_{1}, \ldots, \theta_{n} x_{n}\right)$, etc., for $\theta=\left(\theta_{1}, \ldots, \theta_{n}\right) \in\{-1,1\}^{n}$ and

$$
\tilde{Z}_{ \pm}(s ; \varphi ; f, g)\left(=\tilde{Z}_{ \pm}(s ; \varphi)\right):=\int_{\mathbb{R}_{+}^{n}} f(x)_{ \pm}^{s} g(x) \varphi(x) d x .
$$

Consider the case when $g(x)=x^{p} \psi(x)$. Since $g_{\theta}(x)=\theta^{p} x^{p} \psi_{\theta}(x)$, we have

$$
Z_{ \pm}(s ; \varphi)=\sum_{\theta \in\{-1,1\}^{n}} \theta^{p} \hat{Z}_{ \pm}\left(s ; \varphi_{\theta} ; f_{\theta}, \psi_{\theta}\right),
$$

where $\theta^{p}:=\prod_{j=1}^{n} \theta_{j}^{p_{j}}$ and

$$
\hat{Z}_{ \pm}(s ; \varphi ; f, \psi):=\int_{\mathbb{R}_{+}^{n}} f(x)_{ \pm}^{s} \psi(x) \varphi(x) x^{p} d x .
$$

We remark that $\tilde{Z}_{ \pm}(s ; \varphi ; f, g)=\hat{Z}_{ \pm}(s ; \varphi ; f, \psi)$, while $\tilde{Z}_{ \pm}\left(s ; \varphi_{\theta} ; f_{\theta}, g_{\theta}\right)=$ $-\hat{Z}_{ \pm}\left(s ; \varphi_{\theta} ; f_{\theta}, \psi_{\theta}\right)$ if $\theta^{p}=-1$. From the equations (10.3), (10.5), it suffices to prove the theorem in the case of $\tilde{Z}_{ \pm}(s ; \varphi ; f, g)$ or $\hat{Z}_{ \pm}(s ; \varphi ; f, \psi)$ instead of $Z_{ \pm}(s ; \varphi)$.

Step 2 (Decompositions of $\tilde{Z}_{ \pm}(s ; \varphi)$ ). For the moment, we assume $\operatorname{Re}(s)>0$. By using the mapping $x=\pi(y), \tilde{Z}_{ \pm}(s ; \varphi)$ can be expressed as

$$
\begin{aligned}
\tilde{Z}_{ \pm}(s ; \varphi)=\int_{\mathbb{R}_{+}^{n}} f(x)_{ \pm}^{s} g(x) \varphi(x) d x \\
\quad=\int_{\tilde{Y}_{\Sigma}}((f \circ \pi)(y))_{ \pm}^{s}(g \circ \pi)(y)(\varphi \circ \pi)(y)\left|J_{\pi}(y)\right| d y,
\end{aligned}
$$

where $\tilde{Y}_{\Sigma}=Y_{\Sigma} \cap \pi^{-1}\left(\mathbb{R}_{+}^{n}\right)$ and $d y$ is a volume element in $Y_{\Sigma}$. It is easy to see that there exists a set of $C_{0}^{\infty}$ functions $\left\{\chi_{\sigma}: Y_{\Sigma} \rightarrow \mathbb{R}_{+}: \sigma \in \Sigma^{(n)}\right\}$ satisfying the following properties:

- For each $\sigma \in \Sigma^{(n)}$, the support of the function $\chi_{\sigma}$ is contained in $\mathbb{R}^{n}(\sigma)$, and $\chi_{\sigma}$ identically equals one in some neighborhood of the origin.

- $\sum_{\sigma \in \Sigma^{(n)}} \chi_{\sigma} \equiv 1$ on the support of $\varphi \circ \pi$.

Applying Lemmas 5.1 and 7.1 and Theorem 7.2 we have

$$
\tilde{Z}_{ \pm}(s ; \varphi)=\sum_{\sigma \in \Sigma^{(n)}} Z_{ \pm}^{(\sigma)}(s)
$$


with

$$
\begin{aligned}
& Z_{ \pm}^{(\sigma)}(s):=\int_{\mathbb{R}_{+}^{n}}((f \circ \pi(\sigma))(y))_{ \pm}^{s}(g \circ \pi(\sigma))(y)(\varphi \circ \pi(\sigma))(y) \chi_{\sigma}(y)\left|J_{\pi(\sigma)}(y)\right| d y \\
& =\int_{\mathbb{R}_{+}^{n}}\left(\prod_{j=1}^{n} y_{j}^{l_{f}\left(a^{j}(\sigma)\right)} f_{\sigma}(y)\right)_{ \pm}^{s}\left(\prod_{j=1}^{n} y_{j}^{\left\langle a^{j}(\sigma), p\right\rangle}\right)\left|\prod_{j=1}^{n} y_{j}^{\left\langle a^{j}(\sigma)\right\rangle-1}\right| \tilde{\chi}_{\sigma}(y) d y,
\end{aligned}
$$

where $\tilde{\chi}_{\sigma}(y)=\psi(\pi(\sigma)(y)) \varphi(\pi(\sigma)(y)) \chi_{\sigma}(y)$.

Consider the functions $Z_{ \pm}^{(\sigma)}(s)$ for $\sigma \in \Sigma^{(n)}$. We easily see the existence of finite sets of $C_{0}^{\infty}$ functions $\left\{\xi_{k}: \mathbb{R}^{n} \rightarrow \mathbb{R}_{+}\right\}$and $\left\{\eta_{l}: \mathbb{R}^{n} \rightarrow \mathbb{R}_{+}\right\}$satisfying the following conditions.

- The supports of $\xi_{k}$ and $\eta_{l}$ are sufficiently small and $\sum_{k} \psi_{k}+\sum_{l} \eta_{l} \equiv 1$ on the support of $\tilde{\chi}_{\sigma}$.

- For each $k$, the function $f_{\sigma}$ is always positive or negative on the support of $\xi_{k}$.

- For each $l$, the support of $\eta_{l}$ intersects the set $\left\{y \in \operatorname{Supp}\left(\tilde{\chi}_{\sigma}\right): f_{\sigma}(y)=0\right\}$.

- The union of the support of $\eta_{l}$ for all $l$ contains the set $\left\{y \in \operatorname{Supp}\left(\tilde{\chi}_{\sigma}\right)\right.$ : $\left.f_{\sigma}(y)=0\right\}$.

By using the functions $\xi_{k}$ and $\eta_{l}$, we have

$$
Z_{ \pm}^{(\sigma)}(s)=\sum_{k} I_{ \pm, \sigma}^{(k)}(s)+\sum_{l} J_{ \pm, \sigma}^{(l)}(s)
$$

with

$$
\begin{aligned}
& I_{ \pm, \sigma}^{(k)}(s)=\int_{\mathbb{R}_{+}^{n}}\left(\prod_{j=1}^{n} y_{j}^{l_{f}\left(a^{j}(\sigma)\right)} f_{\sigma}(y)\right)_{ \pm}^{s}\left(\prod_{j=1}^{n} y_{j}^{\left\langle a^{j}(\sigma), p\right\rangle}\right) \mid \prod_{j=1}^{n} y_{j}^{\left\langle a^{j}(\sigma)\right\rangle-1 \mid \tilde{\xi}_{k}(y) d y,} \\
& J_{ \pm, \sigma}^{(l)}(s)=\int_{\mathbb{R}_{+}^{n}}\left(\prod_{j=1}^{n} y_{j}^{l_{f}\left(a^{j}(\sigma)\right)} f_{\sigma}(y)\right)_{ \pm}^{s}\left(\prod_{j=1}^{n} y_{j}^{\left\langle a^{j}(\sigma), p\right\rangle}\right) \mid \prod_{j=1}^{n} y_{j}^{\left\langle a^{j}(\sigma)\right\rangle-1 \mid} \tilde{\eta}_{l}(y) d y,
\end{aligned}
$$

where $\tilde{\xi}_{k}(y)=\tilde{\chi}_{\sigma}(y) \xi_{k}(y)$ and $\tilde{\eta}_{l}(y)=\tilde{\chi}_{\sigma}(y) \eta_{l}(y)$. If the set $\left\{y \in \operatorname{Supp}\left(\tilde{\chi}_{\sigma}\right)\right.$ : $\left.f_{\sigma}(y)=0\right\} \cap \mathbb{R}_{+}^{n}$ is empty, then the functions $J_{ \pm, \sigma}^{(l)}(s)$ do not appear.

Step 3 (Poles of $I_{ \pm, \sigma}^{(k)}(s)$ ). First, consider properties of poles of the functions $I_{ \pm, \sigma}^{(k)}(s)$. Since every $y_{j}$ is nonnegative in the integrand, we have

$$
I_{ \pm, \sigma}^{(k)}(s)=\int_{\mathbb{R}_{+}^{n}}\left(\prod_{j=1}^{n} y_{j}^{l_{f}\left(a^{j}(\sigma)\right) s+\left\langle a^{j}(\sigma), p+\mathbf{1}\right\rangle-1}\right) f_{\sigma}(y)_{ \pm}^{s} \tilde{\xi}_{k}(y) d y .
$$

By applying Proposition 9.2 to (10.10), each $I_{ \pm, \sigma}^{(k)}(s)$ can be analytically continued to the whole complex plane as meromorphic functions and their poles are contained in the set

$$
\left\{-\frac{\left\langle a^{j}(\sigma), p+\mathbf{1}\right\rangle+\nu}{l_{f}\left(a^{j}(\sigma)\right)}: \nu \in \mathbb{Z}_{+}, j \in B(\sigma)\right\}
$$

where

$$
B(\sigma):=\left\{j: l_{f}\left(a^{j}(\sigma)\right) \neq 0\right\} \subset\{1, \ldots, n\} .
$$


Step 4 (Poles of $J_{ \pm, \sigma}^{(l)}(s)$ ). Next, consider the case of the functions $J_{ \pm, \sigma}^{(l)}(s)$. By applying Theorem 7.2 and changing the integral variables as in Remark $7.3, J_{ \pm, \sigma}^{(l)}(s)$ can be expressed as follows:

$$
\begin{aligned}
J_{ \pm, \sigma}^{(l)}(s)= & \int_{\mathbb{R}_{+}^{n}}\left(\left(u_{i}-b_{i}\right) \prod_{j \in B_{l}(\sigma)} u_{j}^{l_{f}\left(a^{j}(\sigma)\right)}\right)_{ \pm}^{s}\left(\prod_{j \in B_{l}(\sigma)} u_{j}^{\left\langle a^{j}(\sigma), p\right\rangle}\right) \\
& \times\left|\prod_{j \in B_{l}(\sigma)} u_{j}^{\left\langle a^{j}(\sigma)\right\rangle-1}\right| \hat{\eta}_{l}\left(u_{1}, \ldots, u_{i}-b_{i}, \ldots, u_{n}\right) d u
\end{aligned}
$$

where $B_{l}(\sigma) \subsetneq\{1, \ldots, n\}, i \in\{1, \ldots, n\} \backslash B_{l}(\sigma), b_{i}>0$ and $\hat{\eta}_{l} \in C_{0}^{\infty}\left(\mathbb{R}^{n}\right)$ has a support containing the origin. In a similar fashion to the case of $I_{ \pm, \sigma}^{(k)}(s)$, we have $(10.14)$

$$
J_{ \pm, \sigma}^{(l)}(s)=\int_{\mathbb{R}_{+}^{n}}\left(u_{i}^{s} \prod_{j \in B_{l}(\sigma)} u_{j}^{l_{f}\left(a^{j}(\sigma)\right) s+\left\langle a^{j}(\sigma), p+\mathbf{1}\right\rangle-1}\right) \hat{\eta}_{l}\left(u_{1}, \ldots, \pm u_{i}, \ldots, u_{n}\right) d u .
$$

By applying Proposition 9.2 to (10.14), each $J_{ \pm, \sigma}^{(l)}(s)$ can be analytically continued to the complex plane as meromorphic functions and their poles are contained in the set

$$
\left\{-\frac{\left\langle a^{j}(\sigma), p+\mathbf{1}\right\rangle+\nu}{l_{f}\left(a^{j}(\sigma)\right)}: \nu \in \mathbb{Z}_{+}, j \in \tilde{B}_{l}(\sigma)\right\} \cup(-\mathbb{N})
$$

where $\tilde{B}_{l}(\sigma):=\left\{j \in B_{l}(\sigma): l_{f}\left(a^{j}(\sigma)\right) \neq 0\right\}$.

From the relationships (10.6) and (10.8), the analyses in Steps 3 and 4 imply that $Z_{ \pm}(s)$ also become meromorphic functions on $\mathbb{C}$ and their poles are contained in the union of the sets (10.11) and (10.15) for all $\sigma \in \Sigma^{(n)}$, which is assertion (a) in the theorem.

Now, for $p \in \mathbb{Z}_{+}^{n}$, we define

$$
\beta(p):=\max \left\{-\frac{\langle a, p+\mathbf{1}\rangle}{l_{f}(a)}: a \in \tilde{\Sigma}^{(1)}\right\} .
$$

Note that $\beta(p)$ is the largest element of the first set in (10.1).

Step 5 (Geometrical meanings of $\beta(p)$ ). Assertion (b) in the theorem follows from the following lemma, which reveals the relationship between "the values of $\beta(p)$ " and "the geometrical conditions of $\Gamma_{+}(f)$ and $p$ ".

Lemma 10.4. Let $q=\left(q_{1}, \ldots, q_{n}\right)$ be the point of the intersection of $\partial \Gamma_{+}(f)$ with the line joining the origin and the point $p+\mathbf{1}=\left(p_{1}+1, \ldots, p_{n}+1\right)$. Then

$$
\beta(p)=-\frac{p_{1}+1}{q_{1}}=\cdots=-\frac{p_{n}+1}{q_{n}}=-\frac{\langle p\rangle+n}{\langle q\rangle}=-\frac{1}{d\left(f, x^{p}\right)} .
$$

In particular, we have $q=d\left(f, x^{p}\right)(p+\mathbf{1})$.

Proof. For $a \in \Sigma^{(1)}$, we denote by $q(a)$ the point of the intersection of the hyperplane $H\left(a, l_{f}(a)\right)$ with the line $\{t \cdot(p+\mathbf{1}): t \in \mathbb{R}\}$, where $H(\cdot, \cdot)$ is as in (2.1). Then it is easy to see that

$$
q(a)=\frac{l_{f}(a)}{\langle a, p+\mathbf{1}\rangle} \cdot(p+\mathbf{1})
$$


From (10.17), the condition that $-\langle a, p+\mathbf{1}\rangle / l_{f}(a)$ takes the maximum is equivalent to the geometrical condition that $q(a)$ is as far as possible from the origin. To be more precise, we have the following equivalences: For $a \in \tilde{\Sigma}^{(1)}$,

$$
\beta(p)=-\frac{\langle a, p+\mathbf{1}\rangle}{l_{f}(a)} \Longleftrightarrow q=q(a) \Longleftrightarrow q \in H\left(a, l_{f}(a)\right) .
$$

From (10.17) and (10.18), we have $-\beta(p)=\left(p_{1}+1\right) / q_{1}=\cdots=\left(p_{n}+1\right) / q_{n}=$ $(\langle p\rangle+n) /\langle q\rangle$. From the definition of $d(\cdot, \cdot)$, the above value equals $1 / d\left(f, x^{p}\right)$.

Step 6 (Orders of the poles at $\beta(p)$ ). Let us consider the orders of the poles of $\tilde{Z}_{ \pm}(s ; \varphi)$ at $s=\beta(p)$. For $\sigma \in \Sigma^{(n)}$, let

$$
A_{p}(\sigma):=\left\{j \in B(\sigma): \beta(p)=-\frac{\left\langle a^{j}(\sigma), p+\mathbf{1}\right\rangle}{l_{f}\left(a^{j}(\sigma)\right)}\right\} \subset\{1, \ldots, n\},
$$

where $B(\sigma)$ is as in (10.12). From (10.6), (10.8), it suffices to analyze the poles of $I_{ \pm, \sigma}^{(k)}(s)$ and $J_{ \pm, \sigma}^{(l)}(s)$. Applying Proposition 9.2 to the integrals (10.10) and (10.14), we see the upper bounds of orders of the poles at $s=\beta(p)$ of $I_{ \pm, \sigma}^{(k)}(s)$ and $J_{ \pm, \sigma}^{(l)}(s)$ as follows:

\begin{tabular}{c|c}
\hline$I_{ \pm, \sigma}^{(k)}(s)$ & $\# A_{p}(\sigma)$ \\
\hline$J_{ \pm, \sigma}^{(l)}(s)$ & $\begin{array}{l}\min \left\{\# A_{p}(\sigma), n-1\right\} \text { if } \beta(p) \notin(-\mathbb{N}) \\
\min \left\{\# A_{p}(\sigma)+1, n\right\} \text { if } \beta(p) \in(-\mathbb{N})\end{array}$ \\
\hline
\end{tabular}

Assertion (c) in the theorem follows from the above table and Lemma10.5 below, which show the geometrical meaning of $\# A_{p}(\sigma)$. (The estimate $\# A_{p}(\sigma) \leq m\left(f, x^{p}\right)$ is sufficient for the proof of assertion (c). The existence of $\sigma \in \Sigma^{(n)}$ attaining the equality will be used in the analysis in the next subsection.)

Lemma 10.5. Let $q$ be as in Lemma 10.4. Then the equations $m\left(f, x^{p}\right)=n-$ $\operatorname{dim}\left(\tau_{f}(q)\right)=\max \left\{\# A_{p}(\sigma): \sigma \in \Sigma^{(n)}\right\}$ hold.

Proof. In the case when $g(x)=x^{p}$, we have $\Gamma_{+}(g)=\{p\}+\mathbb{R}_{+}^{n}$. Since each nonempty proper face of $\Gamma_{+}(g)$ contains the vertex $p$, there exists only one principal face $\gamma_{*}$ of $\Gamma_{+}(g)$ and $p$ is contained in $\gamma_{*}$, which implies that $n-\operatorname{dim}\left(\tau_{f}(q)\right)=m\left(f, x^{p}\right)$. From the definition of $A_{p}(\sigma)$ and (10.18), we have

$$
\begin{aligned}
& A_{p}(\sigma)=\left\{j: q \in H\left(a^{j}(\sigma), l_{f}\left(a^{j}(\sigma)\right)\right)\right\} \\
& \quad=\left\{j: \tau_{f}(q) \subset H\left(a^{j}(\sigma), l_{f}\left(a^{j}(\sigma)\right)\right)\right\}=I\left(\tau_{f}(q), \sigma\right),
\end{aligned}
$$

where $I(\cdot, \cdot)$ is as in (6.5). Lemma 6.1 (ii) implies that $\operatorname{dim}\left(\tau_{f}(q)\right) \leq n-\# I\left(\tau_{f}(q), \sigma\right)$ $=n-\# A_{p}(\sigma)$ for any $\sigma \in \Sigma^{(n)}$. On the other hand, Lemma 6.2 (ii) implies that $\Sigma^{(n)}\left(\tau_{f}(q)\right) \neq \emptyset$; i.e., there exists $\sigma \in \Sigma^{(n)}$ such that $\operatorname{dim}\left(\tau_{f}(q)\right)=n-\# A_{p}(\sigma)$.

10.2. First coefficients. Next, let us consider the coefficients of the most important term of the Laurent expansions of $\tilde{Z}_{ \pm}(s ; \varphi)$.

We define the subset $\Sigma_{p}^{(n)}$ in $\Sigma^{(n)}$ consisting of important cones as

$$
\Sigma_{p}^{(n)}:=\left\{\sigma \in \Sigma^{(n)}: \# A_{p}(\sigma)=m\left(f, x^{p}\right)\right\} .
$$


It follows from Lemma 10.5 that $\Sigma_{p}^{(n)}$ is nonempty. From the definition of $m\left(f, x^{p}\right)$ and Lemma 6.2, we can see the following:

$$
\begin{aligned}
& \sigma \in \Sigma_{p}^{(n)} \\
\Longleftrightarrow & \operatorname{dim}\left(\tau_{f}(q)\right)=n-m\left(f, x^{p}\right)=n-\# A_{p}(\sigma)=n-\# I\left(\tau_{f}(q), \sigma\right) \\
\Longrightarrow & \tau_{f}(q)=\bigcap_{j \in A_{p}(\sigma)} H\left(a^{j}(\sigma), l\left(a^{j}(\sigma)\right)\right) \cap \Gamma_{+}(f),
\end{aligned}
$$

where $q:=d\left(f, x^{p}\right)(p+\mathbf{1})$. Note the equation $A_{p}(\sigma)=I\left(\tau_{f}(q), \sigma\right)$ in the proof of Lemma 10.5. Hence, the equation $\gamma(I, \sigma)=\gamma$ holds for $\sigma \in \Sigma_{p}^{(n)}, I=A_{p}(\sigma)$ and $\gamma=\tau_{f}(q)$, which was an important condition in Lemma 7.1.

Let us compute the coefficients of $(s-\beta(p))^{-m\left(f, x^{p}\right)}$ in the Laurent expansions of $\tilde{Z}_{ \pm}(s ; \varphi)$. Respectively, we define

$$
\tilde{C}_{ \pm}:=\lim _{s \rightarrow \beta(p)}(s-\beta(p))^{m\left(f, x^{p}\right)} \tilde{Z}_{ \pm}(s ; \varphi) .
$$

Proposition 10.6. Suppose that (i) $f$ satisfies the condition (E), (ii) $g(x)=$ $x^{p} \psi(x)$, where $p \in \mathbb{Z}_{+}^{n}$ and $\psi$ is a smooth function defined on $U$ and (iii) at least one of the following conditions is satisfied.

(a) $d\left(f, x^{p}\right)>1$

(b) $f_{\sigma} \circ T_{A_{p}(\sigma)}$ does not vanish on $\mathbb{R}_{+}^{n} \cap \pi(\sigma)^{-1}(U)$ for any $\sigma \in \Sigma_{p}^{(n)}$.

Then we give explicit formulae for coefficients $\tilde{C}_{ \pm}=G_{ \pm}(f, \psi, \varphi)$, where $G_{ \pm}(f, \psi, \varphi)$ are as in (10.26), (10.29), (10.30), (10.31) in the proof of this proposition.

Proof. In this proof, we use the following notation and symbols to decrease the complexity in the expressions of the integrals below.

- $\prod_{j \notin A_{p}(\sigma)} y_{j}^{a_{j}} d y_{j}$ means $\prod_{j \notin A_{p}(\sigma)} y_{j}^{a_{j}} \cdot \prod_{j \notin A_{p}(\sigma)} d y_{j}$ with $a_{j} \geq 0$.

- $L_{\sigma}:=\prod_{j \in A_{p}(\sigma)} l_{f}\left(a^{j}(\sigma)\right)^{-1}$.

- $M_{j}(\sigma):=-l_{f}\left(a^{j}(\sigma)\right) / d\left(f, x^{p}\right)+\left\langle a^{j}(\sigma), p+\mathbf{1}\right\rangle$.

- If $a=0$, then the value of $a^{-1 / d\left(f, x^{p}\right)}$ is defined by 0 .

- $((\psi \cdot \varphi) \circ \pi(\sigma))\left(T_{A}(y)\right):=(\psi \circ \pi(\sigma))\left(T_{A}(y)\right) \cdot(\varphi \circ \pi(\sigma))\left(T_{A}(y)\right)$.

Note that $M_{j}(\sigma)$ is a nonnegative constant and, moreover, $M_{j}(\sigma)=0$ if and only if $j \in A_{p}(\sigma)$.

We divide the computation into the following two cases: $m\left(f, x^{p}\right)<n$ and $m\left(f, x^{p}\right)=n$.

The case: $m\left(f, x^{p}\right)<n$.

Under the hypothesis (a). First, we consider the case when the hypothesis (a),

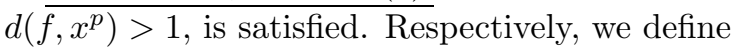

$$
\tilde{C}_{ \pm}(\sigma):=\lim _{s \rightarrow \beta(p)}(s-\beta(p))^{m\left(f, x^{p}\right)} Z_{ \pm}^{(\sigma)}(s),
$$

where $Z_{ \pm}^{(\sigma)}(s)$ are as in (10.7). If $\sigma \notin \Sigma_{p}^{(n)}$, then $\tilde{C}_{ \pm}(\sigma)=0$. Thus, it suffices to consider the case when $\sigma \in \Sigma_{p}^{(n)}$. Considering the equations (10.8) and applying Proposition 9.3 to (10.10) and (10.14) with $A_{*}=A_{p}(\sigma)$, we have

$$
\tilde{C}_{ \pm}(\sigma)=\sum_{k} G_{k, \pm}(\sigma)+\sum_{l} H_{l, \pm}(\sigma)
$$


with

$$
G_{k, \pm}(\sigma)=L_{\sigma} \int_{\left.\mathbb{R}_{+}^{n-m(f, x} p\right)} \frac{\tilde{\xi}_{k}\left(T_{A_{p}(\sigma)}(y)\right)}{f_{\sigma}\left(T_{A_{p}(\sigma)}(y)\right)_{ \pm}^{1 / d\left(f, x^{p}\right)}} \prod_{j \notin A_{p}(\sigma)} y_{j}^{M_{j}(\sigma)-1} d y_{j}
$$

and

$$
\begin{aligned}
& H_{l, \pm}(\sigma) \\
& =L_{\sigma} \int_{\mathbb{R}_{+}^{n-m\left(f, x^{p}\right)}} \frac{\hat{\eta}_{l}\left(T_{A_{p}(\sigma)}\left(u_{1}, \ldots, \pm u_{i}, \ldots, u_{n}\right)\right)}{u_{i}^{1 / d\left(f, x^{p}\right)}} \prod_{j \in B_{l}(\sigma) \backslash A_{p}(\sigma)} u_{j}^{M_{j}(\sigma)-1} \prod_{j \notin A_{p}(\sigma)} d u_{j},
\end{aligned}
$$

where $\tilde{\xi}_{k}, \hat{\eta}_{l}, B_{l}(\sigma), i$ are as in (10.10), (10.14). The summations in (10.22) are taken for all $k, l$ satisfying $T_{A_{p}(\sigma)}\left(\mathbb{R}^{n}\right) \cap \operatorname{Supp}\left(\xi_{k}\right) \neq \emptyset$ and $A_{p}(\sigma) \subset B_{l}(\sigma)$, respectively. We remark that the values of $G_{k, \pm}(\sigma)$ and $H_{l, \pm}(\sigma)$ depend on the cut-off functions $\chi_{\sigma}, \xi_{k}, \eta_{l}$ in Section 10.1. Since $d\left(f, x^{p}\right)>1$, the integrals in (10.23), (10.24) are convergent and they are interpreted as improper integrals.

In (10.23), (10.24), we deform the cut-off functions $\xi_{k}$ and $\eta_{l}$ as the volume of the support of $\eta_{l}$ tends to zero for all $l$. Then, it is easy to see that the limit of $H_{l, \pm}(\sigma)$ is zero, while that of $\sum_{k} G_{k, \pm}(\sigma)$ can be computed explicitly. Considering the equation (10.22) and the deformation of $\xi_{k}$ and $\eta_{l}$ in the above, we have

$$
\tilde{C}_{ \pm}(\sigma)=L_{\sigma} \int_{\mathbb{R}_{+}^{n-m\left(f, x^{p}\right)}} \frac{\tilde{\chi}_{\sigma}\left(T_{A_{p}(\sigma)}(y)\right)}{\left(f_{\sigma}\left(T_{A_{p}(\sigma)}(y)\right)\right)_{ \pm}^{1 / d\left(f, x^{p}\right)}} \prod_{j \notin A_{p}(\sigma)} y_{j}^{M_{j}(\sigma)-1} d y_{j},
$$

where $\tilde{\chi}_{\sigma}$ is as in (10.7).

Furthermore, let us compute the limits $\tilde{C}_{ \pm}$explicitly. If the cut-off function $\chi_{\sigma}$ is deformed as the volume of the support of $\chi_{\sigma}$ tends to zero, then $\tilde{C}_{ \pm}(\sigma)$ tends to zero. Notice that each $\mathbb{R}_{+}^{n}(\sigma)$ is densely embedded in $\tilde{Y}_{\Sigma}$ (see Section [5.2) and that $\tilde{C}_{ \pm}=\sum_{\sigma \in \Sigma_{p}^{(n)}} \tilde{C}_{ \pm}(\sigma)$. Thus, for an arbitrary fixed cone $\sigma \in \Sigma_{p}^{(n)}$, we have $\tilde{C}_{ \pm}=G_{ \pm}(f, \psi, \varphi)$ with

$$
G_{ \pm}(f, \psi, \varphi)=L_{\sigma} \int_{\mathbb{R}_{+}^{n-m\left(f, x^{p}\right)}} \frac{((\psi \cdot \varphi) \circ \pi(\sigma))\left(T_{A_{p}(\sigma)}(y)\right)}{\left(f_{\sigma}\left(T_{A_{p}(\sigma)}(y)\right)\right)_{ \pm}^{1 / d\left(f, x^{p}\right)}} \prod_{j \notin A_{p}(\sigma)} y_{j}^{M_{j}(\sigma)-1} d y_{j} .
$$

From the above computation, we can see that the value of the above integral must be independent of $\sigma \in \Sigma_{p}^{(n)}$. Since the function $f_{\sigma}$ in (10.26) appears after the process of toric resolution, one should look for the other formulae, which are expressed by $\psi, \varphi, f$ more directly.

Let us give the other formulae of $G_{ \pm}(f, \psi, \varphi)$. From the equation (10.21), Lemma 7.1 implies

$$
\left(f_{\tau_{f}(q)} \circ \pi(\sigma)\right)(y)=\left(\prod_{j=1}^{n} y_{j}^{l_{f}\left(a^{j}(\sigma)\right)}\right) f_{\sigma}\left(T_{A_{p}(\sigma)}(y)\right) .
$$

Moreover, we have

$$
\left(f_{\tau_{f}(q)} \circ \pi(\sigma)\right)\left(T_{A_{p}(\sigma)}^{1}(y)\right)=\left(\prod_{j \notin A_{p}(\sigma)} y_{j}^{l_{f}\left(a^{j}(\sigma)\right)}\right) f_{\sigma}\left(T_{A_{p}(\sigma)}(y)\right),
$$


where $T_{A_{p}(\sigma)}^{1}(\cdot)$ is as in (1.4). By using the above equation, (10.26) can be rewritten as

$$
\begin{aligned}
& G_{ \pm}(f, \psi, \varphi) \\
& =L_{\sigma} \int_{\mathbb{R}_{+}^{n-m(f, x p)}} \frac{((\psi \cdot \varphi) \circ \pi(\sigma))\left(T_{A_{p}(\sigma)}(y)\right)}{\left(\left(f_{\tau_{f}(q)} \circ \pi(\sigma)\right)\left(T_{A_{p}(\sigma)}^{1}(y)\right)\right)_{ \pm}^{1 / d\left(f, x^{p}\right)}} \prod_{j \notin A_{p}(\sigma)} y_{j}^{\left\langle a^{j}(\sigma)\right\rangle-1} d y_{j} .
\end{aligned}
$$

Under the hypothesis (b). Secondly, we consider the case that the hypothesis (b) is satisfied. In this case, we immediately obtain the equations (10.25) for $\sigma \in$ $\Sigma_{p}^{(n)}$ in the same argument as in the case of the hypothesis (a), because $J_{ \pm, \sigma}^{(l)}(s)$ do not appear in the decompositions (10.8) and $f_{\sigma} \circ T_{A_{p}(\sigma)}$ does not vanish. But, in this case, we must be careful that $\tilde{C}_{ \pm}(\sigma)$ does not always vanish even in the case when $\sigma \notin \Sigma_{p}^{(n)}$. Indeed, some $J_{ \pm, \sigma}^{(l)}(s)$ may have at $s=-1 / d\left(f, x^{p}\right)$ a pole of order $m\left(f, x^{p}\right)$ even if $\sigma \notin \Sigma_{p}^{(n)}$. Here, the coefficients $\tilde{C}_{ \pm}(\sigma)$ for $\sigma \notin \Sigma_{p}^{(n)}$ can be computed in a similar argument as in the proof of Lemma 9.1. since $I_{ \pm, \sigma}^{(k)}(s)$ do not have at $s=-1 / d\left(f, x^{p}\right)$ poles of order $m\left(f, x^{p}\right)$. From the result of this computation, it is easy to see that these coefficients tend to zero if the volume of the support of $\chi_{\sigma}$ tends to zero. Therefore, the limits $\tilde{C}_{ \pm}$can be similarly computed as in (10.26) and (10.29), where $\sigma$ is as in the hypothesis (b). We remark that $\tilde{C}_{+}$ or $\tilde{C}_{-}$is equal to zero in this case.

The case: $m\left(f, x^{p}\right)=n$.

In this case, we see that $A_{p}(\sigma)=\{1, \ldots, n\}, m\left(f, x^{p}\right)=n$ and $\tau_{f}(q)=q$. Similar computations give the following expressions corresponding to (10.26):

$$
G_{ \pm}(f, \psi, \varphi)=L \frac{\psi(0) \varphi(0)}{f_{\sigma}(0)_{ \pm}^{1 / d\left(f, x^{p}\right)}}
$$

where $\sigma \in \Sigma_{p}^{(n)}$ and $L=\sum_{\sigma \in \Sigma_{p}^{(n)}} L_{\sigma}$.

From the equation (10.28), we obtain the other expressions corresponding to (10.29):

$$
G_{ \pm}(f, \psi, \varphi)=L \frac{\psi(0) \varphi(0)}{\left(f_{\tau_{f}(q)}(\mathbf{1})\right)_{ \pm}^{1 / d\left(f, x^{p}\right)}}=L \frac{(q !)^{1 / d\left(f, x^{p}\right)} \psi(0) \varphi(0)}{\left(\partial^{q} f(0)\right)_{ \pm}^{1 / d\left(f, x^{p}\right)}},
$$

where $\mathbf{1}=(1, \ldots, 1)$ and $L$ is the same as in (10.30).

Remark 10.7. Let us consider the case when $\tau_{f}(q)$ is compact. From (10.21), Lemma 6.4 implies that $\pi(\sigma)\left(T_{A_{p}(\sigma)}\left(\mathbb{R}^{n}\right)\right)=0$. Therefore, the formulae (10.26), (10.29) can be expressed in simpler forms as follows:

$$
\begin{gathered}
G_{ \pm}(f, \psi, \varphi)=L_{\sigma} \psi(0) \varphi(0) \int_{\left.\mathbb{R}_{+}^{n-m(f, x} p\right)} \frac{\prod_{j \notin A_{p}(\sigma)} y_{j}^{M_{j}(\sigma)-1} d y_{j}}{\left(f_{\sigma}\left(T_{A_{p}(\sigma)}(y)\right)\right)_{ \pm}^{1 / d\left(f, x^{p}\right)}} \\
=L_{\sigma} \psi(0) \varphi(0) \int_{\mathbb{R}_{+}^{n-m\left(f, x^{p}\right)}} \frac{\prod_{j \notin A_{p}(\sigma)} y_{j}^{\left\langle a^{j}(\sigma)\right\rangle-1} d y_{j}}{\left(\left(f_{\tau_{f}(q)} \circ \pi(\sigma)\right)\left(T_{A_{p}(\sigma)}^{1}(y)\right)\right)_{ \pm}^{1 / d\left(f, x^{p}\right)}}
\end{gathered}
$$


Finally, let us compute the coefficients of $(s-\beta(p))^{-m\left(f, x^{p}\right)}$ in the Laurent expansions of $Z_{ \pm}(s ; \varphi), Z(s ; \varphi)$. Respectively, we define

$$
C_{ \pm}:=\lim _{s \rightarrow \beta(p)}(s-\beta(p))^{m\left(f, x^{p}\right)} Z_{ \pm}(s ; \varphi), \quad C:=\lim _{s \rightarrow \beta(p)}(s-\beta(p))^{m\left(f, x^{p}\right)} Z(s ; \varphi) .
$$

Theorem 10.8. Suppose that (i) $f$ satisfies the condition (E), (ii) $g(x)=x^{p} \psi(x)$, where $p \in \mathbb{Z}_{+}^{n}$ and $\psi$ is a smooth function defined on $U$ and (iii) at least one of the following conditions is satisfied.

(a) $d(f, g)=d\left(f, x^{p}\right)>1$;

(b) $f$ is nonnegative or nonpositive on $U$;

(c) $f_{\tau_{*}}$ does not vanish on $(\mathbb{R} \backslash\{0\})^{n}$, where $\tau_{*}=\tau_{f}(q)$ is the principal face of $\Gamma_{+}(f)$.

Then we give explicit formulae for the coefficients in the following:

$$
C_{ \pm}=\left(\prod_{j=1}^{n} \theta_{j}^{p_{j}}\right) \sum_{\theta \in\{-1,1\}^{n}} G_{ \pm}\left(f_{\theta}, \psi_{\theta}, \varphi_{\theta}\right) \quad \text { and } \quad C=C_{+}+C_{-}
$$

where $f_{\theta}(x)=f\left(\theta_{1} x_{1}, \ldots, \theta_{n} x_{n}\right)$, etc., and $G_{ \pm}(f, \psi, \varphi)$ are as in (10.26), (10.29), (10.30), (10.31).

Furthermore, the formulae (10.32) imply the following. Suppose that (iv) every component of $p \in \mathbb{Z}_{+}^{n}$ is even and $(\mathrm{v}) \psi(0) \varphi(0)$ is positive (resp. negative) and $\psi \cdot \varphi$ is nonnegative (resp. nonpositive) on $U$. If the support of $\varphi$ is sufficiently small, then $C_{ \pm}$are nonnegative (resp. nonpositive) and $C=C_{+}+C_{-}$is positive (resp. negative).

Proof. From the equations (10.3), 10.5), in order to obtain the explicit formulae (10.32), we must show every condition in (iii) implies the condition (a) or (b) in Proposition 10.6 (iii).

Since the case (a) is easily shown, we only consider the cases (b) and (c). It suffices to show that when the support of $\varphi$ is contained in a sufficiently small neighborhood of the origin, the conditions (b) and (c) imply the condition $f_{\sigma} \circ T_{A_{p}(\sigma)}$ does not vanish on $\mathbb{R}^{n} \cap \pi(\sigma)^{-1}(U)$ for any $\sigma \in \Sigma_{p}^{(n)}$. Note that the difference from the condition (b) in Proposition 10.6 is only in " $\mathbb{R}^{n} \cap \pi(\sigma)^{-1}(U)$ ". Let us show this by contradiction.

Let us assume that for some $\sigma \in \Sigma_{p}^{(n)}$ there exists a point $b_{0} \in T_{A_{p}(\sigma)}\left(\mathbb{R}^{n}\right) \cap$ $\pi(\sigma)^{-1}(U)$ such that $f_{\sigma}\left(b_{0}\right)=0$. Since $f$ is nondegenerate over $\mathbb{R}$ with respect to its Newton polyhedron, Theorem 7.2 implies that there are points $b_{1}, b_{2} \in$ $T_{A_{p}(\sigma)}\left(\mathbb{R}^{n}\right) \cap \pi(\sigma)^{-1}(U)$ near $b_{0}$ such that $f_{\sigma}\left(b_{1}\right)>0$ and $f_{\sigma}\left(b_{2}\right)<0$. By using the equations (7.3), (10.27), it is easy to see that the conditions (b) and (c) induce the contradiction to the existence of the above points $b_{1}, b_{2}$.

Finally, we show the sign of the values of $C_{ \pm}$and $C=C_{+}+C_{-}$. Since the nonnegativities or the nonpositivities of $C_{ \pm}$can be directly seen from the explicit formulae with conditions (iii)-(v), we only show the nonvanishing of the value of $C=C_{+}+C_{-}$. Since $f_{\sigma}(0)>0$, the equation (10.28) implies that $f_{\tau_{f}(q)} \circ \pi(\sigma) \circ T_{A_{p}(\sigma)}^{1}$ does not identically equal zero near the origin. Therefore, the conditions on $\psi, \varphi$ in (iv),(v) imply the nonvanishing of $C=C_{+}+C_{-}$. 
10.3. Poles on negative integers. Let us consider the poles of $Z_{ \pm}(s ; \varphi)$ at negative integers in more detail.

For $\lambda \in-\mathbb{N}$, define

$$
\begin{aligned}
& A_{\lambda}(\sigma, p):=\left\{j \in B(\sigma) ; l_{f}\left(a^{j}(\sigma)\right) \lambda+\left\langle a^{j}(\sigma), p+\mathbf{1}\right\rangle-1 \in-\mathbb{N}\right\}, \\
& \rho_{\lambda}(p):=\min \left\{\max \left\{\# A_{\lambda}(\sigma, p): \sigma \in \Sigma^{(n)}\right\}, n-1\right\} .
\end{aligned}
$$

The following proposition is concerned with the poles of $Z_{ \pm}(s ; \varphi)$, which are induced by the set of zeros of $f_{\sigma}$ and will be used in the computation of the coefficients of the asymptotic expansion (1.2) of $I(t ; \varphi)$ (see the proof in Section 14.3).

Proposition 10.9. Suppose that (i) $f$ satisfies the condition (E) and (ii) $g(x)=$ $x^{p} \psi(x)$, where $p \in \mathbb{Z}_{+}^{n}$ and $\psi$ is a smooth function defined on $U$. If the support of $\varphi$ is contained in a sufficiently small neighborhood of the origin, then the orders of poles of $Z_{ \pm}(s ; \varphi)$ at $s=\lambda \in-\mathbb{N}$ are not higher than $\rho_{\lambda}(p)+1$. In particular, if $\lambda>-1 / d\left(f, x^{p}\right)$, then these orders are not higher than 1 . Moreover, let $a_{\lambda}^{ \pm}$be the coefficients of $(s-\lambda)^{-\rho_{\lambda}(p)-1}$ in the Laurent expansions of $Z_{ \pm}(s ; \varphi)$ at $s=\lambda$, respectively. Then we have $a_{\lambda}^{+}=(-1)^{\lambda-1} a_{\lambda}^{-}$for $\lambda \in-\mathbb{N}$.

Proof. By observing Steps 2 and 4 in the proof of Theorem 10.1 it suffices to investigate the poles of each $J_{ \pm, \sigma}^{(l)}(s)$. Applying Lemma 9.4 to (10.14), we obtain the theorem.

\subsection{Generalization of Varchenko's results to the Puiseux series case.}

In this subsection, we introduce a new class of functions which admit asymptotic expansions at the origin of the form of a fractional power series and generalize the results about unweighted local zeta type functions (8.1), (8.2) with $g \equiv 1$ due to Varchenko [30] and Kamimoto and Nose [20, in the case when $f$ belongs to this class. This generalization can be easily done by using the results in the monomial weighted case.

Hereafter in this subsection, let $U$ be a small open neighborhood of the origin in $\mathbb{R}^{n}$. We denote by $U_{+}$the restriction of $U$ to $\mathbb{R}_{+}^{n}$.

Definition 10.10. Let $p=\left(p_{1}, \ldots, p_{n}\right) \in \mathbb{N}^{n}$. We write $\alpha / p:=\left(\alpha_{1} / p_{1}, \ldots, \alpha_{n} / p_{n}\right)$ and $x^{\alpha / p}:=x_{1}^{\alpha_{1} / p_{1}} \cdots x_{n}^{\alpha_{n} / p_{n}}$ for $\alpha=\left(\alpha_{1}, \ldots, \alpha_{n}\right) \in \mathbb{Z}_{+}^{n}$.

(i) We denote by $C_{1 / p}^{\infty}\left(U_{+}\right)$the set of functions $f$ defined on $U_{+}$for which there exist an open neighborhood $V$ of the origin in $\mathbb{R}^{n}$ and a smooth function $F_{f}$ defined on $V$ satisfying the condition (A) in Section 3 such that $U_{+} \subset$ $\Phi_{1 / p}^{-1}(V)$ and $f=F_{f} \circ \Phi_{1 / p}$ on $U_{+}$, where $\Phi_{1 / p}(x):=\left(x_{1}^{1 / p_{1}}, \ldots, x_{n}^{1 / p_{n}}\right)$.

(ii) We denote by $\hat{\mathcal{E}}_{1 / p}\left(U_{+}\right)$the set of functions $f \in C_{1 / p}^{\infty}\left(U_{+}\right)$such that $F_{f}$ belongs to the class $\hat{\mathcal{E}}(V)$.

(iii) Let $f \in C_{1 / p}^{\infty}\left(U_{+}\right)$. When we write the Taylor series of $F_{f}$ at the origin as $F_{f}(x) \sim \sum_{\alpha \in \mathbb{Z}_{+}^{n}} c_{\alpha} x^{\alpha}$, the formal series

$$
f(x) \sim \sum_{\alpha \in \mathbb{Z}_{+}^{n}} c_{\alpha} x^{\alpha / p}:=\sum_{\alpha \in \mathbb{Z}_{+}^{n}} c_{\alpha} x_{1}^{\alpha_{1} / p_{1}} \cdots x_{n}^{\alpha_{n} / p_{n}}
$$

is called the Puiseux series of $f$ at the origin.

(iv) For $f \in C_{1 / p}^{\infty}\left(U_{+}\right)$, we can naturally generalize the definitions of the Newton polyhedron, the Newton distance and its multiplicity, the principal face in the case of the Puiseux series (10.33), which are similarly denoted by 
$\Gamma_{+}(f), d(f), m(f), \tau_{*}$, respectively. Recall that $d(f), m(f), \tau_{*}$ have been defined in Remark 2.11 in the case when $f \in C^{\infty}(U)$. For a compact face $\gamma$ of $\Gamma_{+}(f)$, the $\gamma$-part $f_{\gamma}$ of $f$ is defined by $f_{\gamma}(x):=\sum_{\alpha / p \in \gamma} c_{\alpha} x^{\alpha / p}$. Note that the vertices of the Newton polyhedron $\Gamma_{+}(f)$ are contained in $\mathbb{Q}_{+}^{n}$. (Refer to [6] for the details.)

(v) $f \in C_{1 / p}^{\infty}\left(U_{+}\right)$is said to be nondegenerate over $\mathbb{R}$ with respect to $\Gamma_{+}(f)$ if the $\gamma$-part $f_{\gamma}$ satisfies $\nabla f_{\gamma} \neq(0, \ldots, 0)$ on $\mathbb{R}_{>0}^{n}$ for every compact face $\gamma$ of $\Gamma_{+}(f)$. Note that $f \in C_{1 / p}^{\infty}\left(U_{+}\right)$is smooth in $U_{+} \cap \mathbb{R}_{>0}^{n}$.

We will use the following properties later.

Lemma 10.11. Let $f \in C_{1 / p}^{\infty}\left(U_{+}\right)$. Then we have:

(i) $f$ is nondegenerate over $\mathbb{R}$ with respect to $\Gamma_{+}(f)$ if and only if $F_{f}$ is nondegenerate over $\mathbb{R}$ with respect to $\Gamma_{+}\left(F_{f}\right)$.

(ii) $d\left(F_{f}, x^{p-1}\right)=d(f)$, where $x^{p-1}=\prod_{j=1}^{n} x_{j}^{p_{j}-1}$.

Proof. (i) By using the chain rule, this equivalence can be easily seen.

(ii) From the definitions of the Newton distances $d(\cdot, \cdot)$ and $d(\cdot)$, the geometrical relationship between $\Gamma_{+}\left(F_{f}\right)$ and $\Gamma_{+}(f)$ gives the desired equation.

Now, let us consider the following local zeta type functions in the case when $f$ belongs to the class $C_{1 / p}^{\infty}\left(U_{+}\right)$:

$$
\tilde{Z}_{ \pm}(s ; \varphi)=\int_{\mathbb{R}_{+}^{n}} f(x)_{ \pm}^{s} \varphi(x) d x, \quad \tilde{Z}(s ; \varphi)=\int_{\mathbb{R}_{+}^{n}}|f(x)|^{s} \varphi(x) d x,
$$

where $\varphi$ satisfies the condition (C) in Section 3 .

By applying Theorems 10.1 and 10.8, we obtain the following theorems, whose assertions are almost the same as those in the results in [30, 20] in appearance.

Theorem 10.12. Suppose that $f \in \hat{\mathcal{E}}_{1 / p}\left(U_{+}\right)$is nondegenerate over $\mathbb{R}$ with respect to $\Gamma_{+}(f)$. If the support of $\varphi$ is contained in a sufficiently small neighborhood of the origin, then the functions $\tilde{Z}_{ \pm}(s ; \varphi)$ and $\tilde{Z}(s ; \varphi)$ can be analytically continued as meromorphic functions to the whole complex plane. More precisely, we have the following.

(a) The poles of the functions $\tilde{Z}_{ \pm}(s ; \varphi)$ and $\tilde{Z}(s ; \varphi)$ are contained in the set

$$
\left\{-\frac{\langle a, p\rangle+\nu}{l_{F_{f}}(a)}: \nu \in \mathbb{Z}_{+}, a \in \tilde{\Sigma}^{(1)}\right\} \cup(-\mathbb{N}) \text {. }
$$

Here $l_{F_{f}}(a)$ is as in (7.1), $\Sigma$ is a simplicial subdivision of the fan associated with the Newton polyhedron $\Gamma_{+}\left(F_{f}\right), \Sigma^{(1)}$ is the set of one-dimensional cones in $\Sigma$ and $\tilde{\Sigma}^{(1)}:=\left\{a \in \Sigma^{(1)}: l_{F_{f}}(a) \neq 0\right\}$.

(b) The largest element of the first set in (10.35) is $-1 / d(f)$.

(c) When $\tilde{Z}_{ \pm}(s ; \varphi)$ and $\tilde{Z}(s ; \varphi)$ have poles at $s=-1 / d(f)$, their orders are at most

$$
\begin{cases}m(f) & \text { if } 1 / d(f) \text { is not an integer } \\ \min \{m(f)+1, n\} & \text { otherwise. }\end{cases}
$$


Remark 10.13. It is natural to expect that the set in assertion (a) should be replaced by the following one:

$$
\left\{-\frac{\langle a\rangle+\nu}{l_{f}(a)}: \nu \in \mathbb{Z}_{+}, a \in \tilde{\Sigma}^{(1)}\right\} \cup(-\mathbb{N}) .
$$

Here $l_{f}(a)$ is as in (7.1), $\Sigma$ is a simplicial subdivision of the fan associated with the Newton polyhedron $\Gamma_{+}(f), \Sigma^{(1)}$ is the set of one-dimensional cones in $\Sigma$ and $\tilde{\Sigma}^{(1)}:=\left\{a \in \Sigma^{(1)}: l_{f}(a) \neq 0\right\}$. But we have not yet verified this.

Theorem 10.14. Suppose that $f \in \hat{\mathcal{E}}_{1 / p}\left(U_{+}\right)$is nondegenerate on $\mathbb{R}$ with respect to $\Gamma_{+}(f)$ and that at least one of the following conditions is satisfied:

(a) $d(f)>1$;

(b) $f$ is nonnegative or nonpositive on $U_{+}$;

(c) $f_{\tau_{*}}$ does not vanish on $\mathbb{R}_{>0}^{n}$, where $\tau_{*}$ is the principal face of $\Gamma_{+}(f)$.

Then if $\varphi(0)$ is positive (resp. negative) and $\varphi$ is nonnegative (resp. nonpositive) on $U$ and the support of $\varphi$ is sufficiently small, then $\tilde{C}_{ \pm}$are nonnegative (resp. nonpositive) and $\tilde{C}=\tilde{C}_{+}+\tilde{C}_{-}$is positive (resp. negative), where

$\tilde{C}_{ \pm}:=\lim _{s \rightarrow-1 / d(f)}(s+1 / d(f))^{m(f)} \tilde{Z}_{ \pm}(s ; \varphi), \quad \tilde{C}:=\lim _{s \rightarrow-1 / d(f)}(s+1 / d(f))^{m(f)} \tilde{Z}(s ; \varphi)$.

Proof of Theorems 10.12 and 10.14. We only show the case of $\tilde{Z}_{ \pm}(s ; \varphi)$. Substituting $x=\Phi_{1 / p}^{-1}(y)=\left(y_{1}^{p_{1}}, \ldots, y_{n}^{p_{n}}\right)$ into the integrals in (10.34), we have

$$
\tilde{Z}_{ \pm}(s ; \varphi)=\int_{\mathbb{R}_{+}^{n}} f(x)_{ \pm}^{s} \varphi(x) d x=\left(\prod_{j=1}^{n} p_{j}\right) \int_{\mathbb{R}_{+}^{n}} F_{f}(y)_{ \pm}^{s} \varphi\left(\Phi_{1 / p}^{-1}(y)\right) y^{p-1} d y
$$

where $y^{p-1}=\prod_{j=1}^{n} y_{j}^{p_{j}-1}$. Noticing the nondegeneracy condition for $F_{f}$ from Lemma 10.11 (i) and the equation $d\left(F_{f}, x^{p-\mathbf{1}}\right)=d(f)$ in Lemma 10.11 (ii), we can apply Theorems 10.1 and 10.8 to the integrals in (10.38). As a result, we can obtain the above theorems.

\section{The CASE OF $\hat{\mathcal{E}}$-Weight}

In this section, we more generally investigate the poles of $Z_{ \pm}(s ; \varphi)$ and $Z(s ; \varphi)$ in the case when the weight $g$ belongs to the class $\hat{\mathcal{E}}(U)$, where $U$ is an open neighborhood of the origin. In order to investigate this case, we must use a fan $\Sigma$ constructed from the two Newton polyhedra $\Gamma_{+}(f)$ and $\Gamma_{+}(g)$.

In this section, we use the following notation.

- $\Sigma_{0}$ is the fan associated with $\Gamma_{+}(f)$ and $\Gamma_{+}(g)$;

- $\Sigma$ is a simplicial subdivision of $\Sigma_{0}$;

- $\left(Y_{\Sigma}, \pi\right)$ is the real resolution associated with $\Sigma$;

- $a^{1}(\sigma), \ldots, a^{n}(\sigma)$ is the skeleton of $\sigma \in \Sigma^{(n)}$, ordered once and for all;

- $J_{\pi}(y)$ is the Jacobian of the mapping of $\pi$.

11.1. Candidate poles. First, let us state our results relating to the positions and the orders of candidate poles of $Z_{ \pm}(s ; \varphi)$ and $Z(s ; \varphi)$ with $\hat{\mathcal{E}}$-weights.

Theorem 11.1. Suppose that (i) $f$ satisfies the condition (E) and (ii) $g$ belongs to the class $\hat{\mathcal{E}}(U)$. If the support of $\varphi$ is contained in a sufficiently small neighborhood of the origin, then the functions $Z_{ \pm}(s ; \varphi)$ and $Z(s ; \varphi)$ can be analytically continued 
as meromorphic functions to the whole complex plane. More precisely, we have the following.

(a) The poles of the functions $Z_{ \pm}(s ; \varphi)$ and $Z(s ; \varphi)$ are contained in the set

$$
\left\{-\frac{l_{g}(a)+\langle a\rangle+\nu}{l_{f}(a)}: \nu \in \mathbb{Z}_{+}, a \in \tilde{\Sigma}^{(1)}\right\} \cup(-\mathbb{N}),
$$

where $l_{f}(a)$ and $l_{g}(a)$ are as in (7.1) and $\tilde{\Sigma}^{(1)}=\left\{a \in \Sigma^{(1)}: l_{f}(a) \neq 0\right\}$.

(b) The largest element of the first set of (11.1) is $-1 / d(f, g)$.

(c) When $Z_{ \pm}(s ; \varphi)$ and $Z(s ; \varphi)$ have poles at $s=-1 / d(f, g)$, their orders are at most

$$
\begin{cases}m(f, g) & \text { if }-1 / d(f, g) \text { is not an integer } \\ \min \{m(f, g)+1, n\} & \text { otherwise. }\end{cases}
$$

Proof. We only consider the case of $Z_{ \pm}(s ; \varphi)$. By applying the orthant decomposition, it suffices to investigate the functions

$$
\tilde{Z}_{ \pm}(s ; \varphi)\left(=\tilde{Z}_{ \pm}(s ; \varphi ; f, g)\right)=\int_{\mathbb{R}_{+}^{n}} f(x)_{ \pm}^{s} g(x) \varphi(x) d x .
$$

From Theorem 7.2 , there exist smooth functions $f_{\sigma}$ and $g_{\sigma}$ such that $f_{\sigma}(0) \cdot g_{\sigma}(0) \neq 0$ and

$$
\begin{aligned}
& f(\pi(\sigma)(y))=\left(\prod_{j=1}^{n} y_{j}^{l_{f}\left(a^{j}(\sigma)\right)}\right) f_{\sigma}(y), \\
& g(\pi(\sigma)(y))=\left(\prod_{j=1}^{n} y_{j}^{l_{g}\left(a^{j}(\sigma)\right)}\right) g_{\sigma}(y) .
\end{aligned}
$$

Using the mapping $x=\pi(y)$ and the cut-off functions $\left\{\chi_{\sigma} ; \sigma \in \Sigma^{(n)}\right\}$ in the proof of Theorem 10.1 and substituting the above equations in (11.2), we have

$$
\begin{aligned}
\tilde{Z}_{ \pm}(s ; \varphi) & =\int_{\tilde{Y}_{\Sigma}}((f \circ \pi)(y))_{ \pm}^{s}(g \circ \pi)(y)(\varphi \circ \pi)(y)\left|J_{\pi}(y)\right| d y \\
& =\sum_{\sigma \in \Sigma^{(n)}} Z_{ \pm}^{(\sigma)}(s)
\end{aligned}
$$

with

$$
\begin{aligned}
& Z_{ \pm}^{(\sigma)}(s)=\int_{\mathbb{R}_{+}^{n}}((f \circ \pi(\sigma))(y))_{ \pm}^{s}(g \circ \pi(\sigma))(y)(\varphi \circ \pi(\sigma))(y) \chi_{\sigma}(y)\left|J_{\pi(\sigma)}(y)\right| d y \\
& =\int_{\mathbb{R}_{+}^{n}}\left(\prod_{j=1}^{n} y_{j}^{l_{f}\left(a^{j}(\sigma)\right)} f_{\sigma}(y)\right)_{ \pm}^{s}\left(\prod_{j=1}^{n} y_{j}^{l_{g}\left(a^{j}(\sigma)\right)} g_{\sigma}(y)\right)\left|\prod_{j=1}^{n} y_{j}^{\left\langle a^{j}(\sigma)\right\rangle-1}\right| \tilde{\chi}_{\sigma}(y) d y \\
& =\int_{\mathbb{R}_{+}^{n}}\left(\prod_{j=1}^{n} y_{j}^{l_{f}\left(a^{j}(\sigma)\right) s+l_{g}\left(a^{j}(\sigma)\right)+\left\langle a^{j}(\sigma)\right\rangle-1}\right) f_{\sigma}(y)_{ \pm}^{s} g_{\sigma}(y) \tilde{\chi}_{\sigma}(y) d y,
\end{aligned}
$$

where $\tilde{\chi}_{\sigma}(y)=(\varphi \circ \pi(\sigma))(y) \chi_{\sigma}(y)$. 
By a similar argument as in the proof of Theorem 10.1 we see that the poles of $Z_{ \pm}^{(\sigma)}(s)$ are contained in the set

$$
\left\{-\frac{l_{g}\left(a^{j}(\sigma)\right)+\left\langle a^{j}(\sigma)\right\rangle+\nu}{l_{f}\left(a^{j}(\sigma)\right)}: \nu \in \mathbb{Z}_{+}, j \in B(\sigma)\right\} \cup(-\mathbb{N}),
$$

where $B(\sigma)=\left\{j: l_{f}\left(a^{j}(\sigma)\right) \neq 0\right\}$. From (11.5), we can see (a) in the theorem.

Next, consider a geometrical meaning of the largest element of the set (11.1). The following lemma implies assertion (b) in the theorem.

\section{Lemma 11.2 .}

$$
\max \left\{-\frac{l_{g}(a)+\langle a\rangle}{l_{f}(a)}: a \in \tilde{\Sigma}^{(1)}\right\}=-\frac{1}{d(f, g)} .
$$

Proof. Using the equations in Remark 2.1, we see the following equivalences:

$$
\begin{aligned}
& \Phi\left(\Gamma_{+}(g)\right) \subset \Gamma_{+}(f) \\
\Longleftrightarrow & d(f, g) \cdot\left(\Gamma_{+}(g)+\mathbf{1}\right) \subset \Gamma_{+}(f) \\
\Longleftrightarrow & d(f, g) \cdot\left(H^{+}\left(a, l_{g}(a)\right)+\mathbf{1}\right) \subset H^{+}\left(a, l_{f}(a)\right) \quad \text { for any } a \in \mathbb{Z}_{+}^{n} \\
\Longleftrightarrow & H^{+}\left(a, d(f, g)\left(l_{g}(a)+\langle a\rangle\right)\right) \subset H^{+}\left(a, l_{f}(a)\right) \quad \text { for any } a \in \mathbb{Z}_{+}^{n} \\
\Longleftrightarrow & d(f, g)\left(l_{g}(a)+\langle a\rangle\right) \geq l_{f}(a) \quad \text { for any } a \in \mathbb{Z}_{+}^{n} \\
\Longleftrightarrow & -\frac{l_{g}(a)+\langle a\rangle}{l_{f}(a)} \leq-\frac{1}{d(f, g)} \quad \text { for any } a \in \mathbb{Z}_{+}^{n} .
\end{aligned}
$$

Moreover, from the construction of the fan $\Sigma$, we see the existence of $a \in \Sigma^{(1)}$ satisfying the equality in the last inequality. Therefore, we have the equation in the lemma.

Finally, let us consider the orders of the poles of $\tilde{Z}_{ \pm}(s ; \varphi)$ at $s=-1 / d(f, g)$. For $\sigma \in \Sigma^{(n)}$, let

$$
A(\sigma):=\left\{j \in B(\sigma):-\frac{1}{d(f, g)}=-\frac{l_{g}\left(a^{j}(\sigma)\right)+\left\langle a^{j}(\sigma)\right\rangle}{l_{f}\left(a^{j}(\sigma)\right)}\right\} \subset\{1, \ldots, n\} .
$$

In order to prove the assertion (c) in the theorem, it suffices to show the estimate $\# A(\sigma) \leq m(f, g)$, which will be shown in Lemma 11.5 (ii) in the next subsection.

Remark 11.3. Carefully observing the equivalences in (11.6), we see that for $\sigma \in$ $\Sigma^{(n)}$,

$$
j \in A(\sigma) \Longleftrightarrow \Phi\left(H\left(a^{j}(\sigma), l_{g}\left(a^{j}(\sigma)\right)\right)\right)=H\left(a^{j}(\sigma), l_{f}\left(a^{j}(\sigma)\right)\right) .
$$

11.2. Properties of the principal faces. In order to investigate more precise properties of the leading poles of $Z_{ \pm}(s ; \varphi)$, we must understand more exact relationships between the cones of the fan $\Sigma$ and the faces of the Newton polyhedra $\Gamma_{+}(f)$ and $\Gamma_{+}(g)$. In this subsection, after our interest focuses essentially on important cones and faces, their properties and relationships are investigated in detail.

Now, let us define a class of important faces of $\Gamma_{+}(f), \Gamma_{+}(g)$. For a cone $\sigma \in \Sigma^{(n)}$ with $A(\sigma) \neq \emptyset$, let $\tau_{*}(\sigma)$ (resp. $\left.\gamma_{*}(\sigma)\right)$ be the face of $\Gamma_{+}(f)$ (resp. $\Gamma_{+}(g)$ ) defined 
by

$$
\begin{aligned}
\tau_{*}(\sigma) & :=\bigcap_{j \in A(\sigma)} H\left(a^{j}(\sigma), l_{f}\left(a^{j}(\sigma)\right)\right) \cap \Gamma_{+}(f), \\
\gamma_{*}(\sigma) & :=\bigcap_{j \in A(\sigma)} H\left(a^{j}(\sigma), l_{g}\left(a^{j}(\sigma)\right)\right) \cap \Gamma_{+}(g) .
\end{aligned}
$$

When $A(\sigma)=\emptyset$, we define $\tau_{*}(\sigma)=\gamma_{*}(\sigma):=\emptyset$. From the construction of the fan $\Sigma$, it is easy to see that $\tau_{*}(\sigma)$ (resp. $\left.\gamma_{*}(\sigma)\right)$ is a nonempty face of $\Gamma_{+}(f)\left(\operatorname{resp} . \Gamma_{+}(g)\right.$ ) if $A(\sigma) \neq \emptyset$.

Remark 11.4. In terms of the symbol (6.4) in Section 6, the above equations can be written as $\tau_{*}(\sigma)\left(\operatorname{resp} \gamma_{*}(\sigma)\right)=\gamma(A(\sigma), \sigma)$ with $P=\Gamma_{+}(f)\left(\operatorname{resp} . P=\Gamma_{+}(g)\right)$.

Lemma 11.5. Suppose that $\tau_{*}$ is a principal face of $\Gamma_{+}(f)$. Then we have the following (see (6.5), (6.6) for the definitions of $I(\cdot, \cdot)$ and $\left.\Sigma^{(n)}(\cdot)\right)$.

(i) $I\left(\tau_{*}, \sigma\right) \subset A(\sigma)$ for any $\sigma \in \Sigma^{(n)}$.

(ii) $\# A(\sigma) \leq m(f, g)$ for any $\sigma \in \Sigma^{(n)}$.

(iii) $\Sigma^{(n)}\left(\tau_{*}\right) \subset \Sigma_{*}^{(n)}:=\left\{\sigma \in \Sigma^{(n)}: \# A(\sigma)=m(f, g)\right\}$.

(iv) $\Sigma_{*}^{(n)} \neq \emptyset$.

Proof. (i) Suppose that $j \in I\left(\tau_{*}, \sigma\right)$, i.e., $\tau_{*} \subset H\left(a^{j}(\sigma), l_{f}\left(a^{j}(\sigma)\right)\right)$. Let $\gamma_{*}$ be the principal face of $\Gamma_{+}(g)$ associated to $\tau_{*}$, i.e. $\Psi_{*}\left(\tau_{*}\right)=\gamma_{*}$. Since $\Phi\left(\gamma_{*}\right) \subset \Psi_{*}^{-1}\left(\gamma_{*}\right)=$ $\tau_{*}$, we have

$$
\begin{aligned}
\tau_{*} \subset H\left(a^{j}(\sigma), l_{f}\left(a^{j}(\sigma)\right)\right) & \Longrightarrow \Phi\left(\gamma_{*}\right) \subset H\left(a^{j}(\sigma), l_{f}\left(a^{j}(\sigma)\right)\right) \\
& \Longleftrightarrow \gamma_{*} \subset \Phi^{-1}\left(H\left(a^{j}(\sigma), l_{f}\left(a^{j}(\sigma)\right)\right)\right) .
\end{aligned}
$$

From an easy property of the map $\Phi$, there exists a positive number $l$ such that $\Phi^{-1}\left(H\left(a^{j}(\sigma), l_{f}\left(a^{j}(\sigma)\right)\right)\right)=H\left(a^{j}(\sigma), l\right)$. Since $\Gamma_{+}(g) \subset \Phi^{-1}\left(H^{+}\left(a^{j}(\sigma), l_{f}\left(a^{j}(\sigma)\right)\right)\right)$ and $\gamma_{*}$ is a nonempty proper face of $\Gamma_{+}(g)$, the definition of $l_{g}(\cdot)$ implies $l=$ $l_{g}\left(a^{j}(\sigma)\right)$. Thus, we have

$$
H\left(a^{j}(\sigma), l_{g}\left(a^{j}(\sigma)\right)\right)=\Phi^{-1}\left(H\left(a^{j}(\sigma), l_{f}\left(a^{j}(\sigma)\right)\right)\right),
$$

which implies that $j \in A(\sigma)$ from the equivalence (11.8) in Remark 11.3

(ii) Since the case when $A(\sigma)=\emptyset$ is obvious, we assume that $A(\sigma) \neq \emptyset$. From the equivalence (11.8) in Remark 11.3, we have

$$
\begin{aligned}
& \Phi\left(\bigcap_{j \in A(\sigma)} H\left(a^{j}(\sigma), l_{g}\left(a^{j}(\sigma)\right)\right)\right) \cap \Gamma_{+}(f)=\bigcap_{j \in A(\sigma)} H\left(a^{j}(\sigma), l_{f}\left(a^{j}(\sigma)\right)\right) \cap \Gamma_{+}(f) \\
& .12) \Longleftrightarrow \Phi\left(\bigcap_{j \in A(\sigma)} H\left(a^{j}(\sigma), l_{g}\left(a^{j}(\sigma)\right)\right) \cap \Phi^{-1}\left(\Gamma_{+}(f)\right)\right)=\tau_{*}(\sigma) .
\end{aligned}
$$

Since $\Phi^{-1}\left(\Gamma_{+}(f)\right) \supset \Gamma_{+}(g)$, the left side of (11.12) contains $\Phi\left(\gamma_{*}(\sigma)\right)$, i.e. $\Phi\left(\gamma_{*}(\sigma)\right)$ $\subset \tau_{*}(\sigma)$. Since $\Phi\left(\gamma_{*}(\sigma)\right)$ is a nonempty set in $\Gamma_{0}(f)$ and is contained in the face $\tau_{*}(\sigma)$ of $\Gamma_{+}(f)$, the definition of $\mathcal{F}_{0}\left[\Gamma_{+}(f)\right]$ implies that there exists a face $\tilde{\tau} \in \mathcal{F}_{0}\left[\Gamma_{+}(f)\right]$ such that $\Phi\left(\gamma_{*}(\sigma)\right) \subset \tilde{\tau} \subset \tau_{*}(\sigma)$. From the definition of $m(f, g)$, we have

$$
\operatorname{dim}\left(\tau_{*}(\sigma)\right) \geq \operatorname{dim}(\tilde{\tau}) \geq n-m(f, g) .
$$


On the other hand, we have

$$
\operatorname{dim}\left(\tau_{*}(\sigma)\right) \leq \operatorname{dim}\left(\bigcap_{j \in A(\sigma)} H\left(a^{j}(\sigma), l_{f}\left(a^{j}(\sigma)\right)\right)\right)=n-\# A(\sigma) .
$$

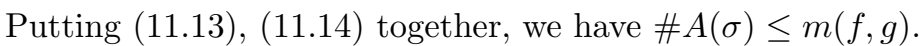

(iii) From the definition (6.6), we have

$$
\Sigma^{(n)}\left(\tau_{*}\right)=\left\{\sigma \in \Sigma^{(n)}: \# I\left(\tau_{*}, \sigma\right)=m(f, g)\right\} .
$$

It follows from (i), (ii) in this lemma that $\Sigma^{(n)}\left(\tau_{*}\right) \subset \Sigma_{*}^{(n)}$.

(iv) Since $\Sigma^{(n)}\left(\tau_{*}\right) \neq \emptyset$ from Lemma 6.2 (ii), we see that $\Sigma_{*}^{(n)} \neq \emptyset$.

Remark 11.6. From Lemma 11.5 (ii), (iv), we see $\max \left\{\# A(\sigma): \sigma \in \Sigma^{(n)}\right\}=$ $m(f, g)$, which is a generalization of Lemma 10.5 .

Proposition 11.7. If $\sigma \in \Sigma_{*}^{(n)}$, then $\tau_{*}(\sigma)$ (resp. $\left.\gamma_{*}(\sigma)\right)$ is a principal face of $\Gamma_{+}(f)$ (resp. $\left.\Gamma_{+}(g)\right)$. Moreover, $\gamma_{*}(\sigma)$ is associated to $\tau_{*}(\sigma)$ (i.e., $\gamma_{*}(\sigma)=$ $\left.\Psi_{*}\left(\tau_{*}(\sigma)\right)\right)$.

Proof. Suppose that $\sigma \in \Sigma_{*}^{(n)}$, i.e., \#A( $\left.\#\right)=m(f, g)$. From (11.13), (11.14), we have $\operatorname{dim}\left(\tau_{*}(\sigma)\right)=n-m(f, g)$ and, moreover, $\tau_{*}(\sigma)=\tilde{\tau} \in \mathcal{F}_{0}\left[\Gamma_{+}(f)\right]$. It follows from these equations that $\tau_{*}(\sigma)$ is a principal face of $\Gamma_{+}(f)$. Moreover, the equation (11.12) implies

$$
\begin{gathered}
\Phi^{-1}\left(\tau_{*}(\sigma)\right)=\bigcap_{j \in A(\sigma)} H\left(a^{j}(\sigma), l_{g}\left(a^{j}(\sigma)\right)\right) \cap \Phi^{-1}\left(\Gamma_{+}(f)\right) \\
\Longrightarrow \Phi^{-1}\left(\tau_{*}(\sigma)\right) \cap \Gamma_{+}(g)=\bigcap_{j \in A(\sigma)} H\left(a^{j}(\sigma), l_{g}\left(a^{j}(\sigma)\right)\right) \cap \Phi^{-1}\left(\Gamma_{+}(f)\right) \cap \Gamma_{+}(g) \\
=\bigcap_{j \in A(\sigma)} H\left(a^{j}(\sigma), l_{g}\left(a^{j}(\sigma)\right)\right) \cap \Gamma_{+}(g)=\gamma_{*}(\sigma) .
\end{gathered}
$$

From the above equations and the definition (2.11), we see that $\gamma_{*}(\sigma)$ is a principal face of $\Gamma_{+}(g)$ associated to $\tau_{*}(\sigma)$.

From Proposition [11.7, the map from $\Sigma_{*}^{(n)}$ to $\mathcal{F}_{*}\left[\Gamma_{+}(f)\right]$ (resp. to $\mathcal{F}_{*}\left[\Gamma_{+}(g)\right]$ ) is naturally defined (i.e., $\sigma \mapsto \tau_{*}(\sigma)$ (resp. $\sigma \mapsto \gamma_{*}(\sigma)$ )).

Lemma 11.8. The above maps are surjective.

Proof. Let $\tau_{*}$ be an arbitrary principal face of $\Gamma_{+}(f)$ and $\sigma$ a cone in $\Sigma^{(n)}\left(\tau_{*}\right)$. Since

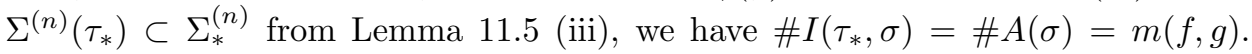
Moreover, since $I\left(\tau_{*}, \sigma\right) \subset A(\sigma)$ from Lemma 11.5 (i), we have $A(\sigma)=I\left(\tau_{*}, \sigma\right)$. As a result, we have

$$
\begin{aligned}
\tau_{*} & =\bigcap_{j \in I\left(\tau_{*}, \sigma\right)} H\left(a^{j}(\sigma), l_{f}\left(a^{j}(\sigma)\right)\right) \cap \Gamma_{+}(f) \\
& =\bigcap_{j \in A(\sigma)} H\left(a^{j}(\sigma), l_{f}\left(a^{j}(\sigma)\right)\right) \cap \Gamma_{+}(f)=\tau_{*}(\sigma) .
\end{aligned}
$$

Note that the first equality follows from Lemma 6.2 (iii) with Remark 11.4, Of course, if $\gamma_{*}$ is a principal face of $\Gamma_{+}(g)$ associated to $\tau_{*}$, then we see that $\gamma_{*}=$ $\Psi_{*}\left(\tau_{*}\right)=\Psi_{*}\left(\tau_{*}(\sigma)\right)=\gamma_{*}(\sigma)$. 
11.3. First coefficients. Let us return to the analysis of the functions $Z_{ \pm}(s ; \varphi)$. First, we compute the coefficients of $(s+1 / d(f, g))^{-m(f, g)}$ in the Laurent expansions of $\tilde{Z}_{ \pm}(s ; \varphi)$. Respectively, we define

$$
\tilde{C}_{ \pm}=\lim _{s \rightarrow-1 / d(f, g)}(s+1 / d(f, g))^{m(f, g)} \tilde{Z}_{ \pm}(s ; \varphi) .
$$

Proposition 11.9. Suppose that (i) $f$ satisfies the condition (E), (ii) $g$ belongs to the class $\hat{\mathcal{E}}(U)$ and (iii) at least one of the following conditions is satisfied:

(a) $d(f, g)>1$;

(b) $f_{\sigma} \circ T_{A(\sigma)}$ does not vanish on $\pi(\sigma)^{-1}(U)$ for any $\sigma \in \Sigma_{*}^{(n)}$.

Then we give explicit formulae for coefficients $\tilde{C}_{ \pm}=G_{ \pm}(f, g, \varphi)$, where $G_{ \pm}(f, g, \varphi)$ are as in (11.19), (11.21), (11.22), (11.23) in the proof of this proposition.

Proof. In this proof, we use the following notation and symbols to decrease the complexity in the integral expression.

- $\prod_{j \notin A(\sigma)} y_{j}^{a_{j}} d y_{j}$ means $\prod_{j \notin A(\sigma)} y_{j}^{a_{j}} \cdot \prod_{j \notin A(\sigma)} d y_{j}$ with $a_{j}>0$.

- $L_{\sigma}:=\prod_{j \in A(\sigma)} l_{f}\left(a^{j}(\sigma)\right)^{-1}$.

- $M_{j}(\sigma):=-l_{f}\left(a^{j}(\sigma)\right) / d(f, g)+l_{g}\left(a^{j}(\sigma)\right)+\left\langle a^{j}(\sigma)\right\rangle$.

- If $a=0$, then the value of $a^{-1 / d(f, g)}$ is defined by 0 .

Note that $M_{j}(\sigma)$ is a nonnegative constant and, moreover, $M_{j}(\sigma)=0$ if and only if $j \in A(\sigma)$.

Let us compute the limits $\tilde{C}_{ \pm}$exactly. We divide the computation into the following two cases: $m(f, g)<n$ and $m(f, g)=n$.

The case: $m(f, g)<n$.

Under the hypothesis (a). First, we consider the case that the hypothesis (a), $d(\overline{f, g)}>1$, is satisfied. Let us explicitly compute the following limits:

$$
\tilde{C}_{ \pm}(\sigma):=\lim _{s \rightarrow-1 / d(f, g)}(s+1 / d(f, g))^{m(f, g)} Z_{ \pm}^{(\sigma)}(s),
$$

where $Z_{ \pm}^{(\sigma)}(s)$ are as in (11.4). We only consider the case that

$$
\sigma \in \Sigma_{*}^{(n)}=\left\{\sigma \in \Sigma^{(n)}: \# A(\sigma)=m(f, g)\right\}
$$

since $\tilde{C}_{ \pm}(\sigma)=0$ if $\sigma \notin \Sigma_{*}^{(n)}$. We remark that $\Sigma_{*}^{(n)}$ is not empty from Lemma 11.5 (iv). By applying the argument as in the proof of Theorem 10.1 and Proposition 10.6 to $Z_{ \pm}^{(\sigma)}(s)$ (compare with $Z_{ \pm}^{(\sigma)}(s)$ defined in (10.7)), we see that if $\sigma \in \Sigma_{*}^{(n)}$, then

$$
\tilde{C}_{ \pm}(\sigma)=L_{\sigma} \int_{\mathbb{R}_{+}^{n-m(f, g)}} \frac{g_{\sigma}\left(T_{A(\sigma)}(y)\right) \tilde{\chi}_{\sigma}\left(T_{A(\sigma)}(y)\right)}{f_{\sigma}\left(T_{A(\sigma)}(y)\right)_{ \pm}^{1 / d(f, g)}} \prod_{j \notin A(\sigma)} y_{j}^{M_{j}(\sigma)-1} d y_{j} .
$$

Furthermore, let us compute the limits $\tilde{C}_{ \pm}$explicitly. If the cut-off function $\chi_{\sigma}$ is deformed as the volume of the support of $\chi_{\sigma}$ tends to zero, then $\tilde{C}_{ \pm}(\sigma)$ tends to zero. Notice that each $\mathbb{R}_{+}^{n}(\sigma)$ is densely embedded in $\tilde{Y}_{\Sigma}$ (see Section [5.2) and that $\tilde{C}_{ \pm}=\sum_{\sigma \in \Sigma_{*}^{(n)}} \tilde{C}_{ \pm}(\sigma)$. Thus, for an arbitrary fixed cone $\sigma \in \Sigma_{*}^{(n)}$, we have

$$
\tilde{C}_{ \pm}=G_{ \pm}(f, g, \varphi)
$$


with

$$
\begin{aligned}
& G_{ \pm}(f, g, \varphi) \\
& =L_{\sigma} \int_{\mathbb{R}_{+}^{n-m(f, g)}} \frac{g_{\sigma}\left(T_{A(\sigma)}(y)\right)(\varphi \circ \pi(\sigma))\left(T_{A(\sigma)}(y)\right)}{f_{\sigma}\left(T_{A(\sigma)}(y)\right)_{ \pm}^{1 / d(f, g)}} \prod_{j \notin A(\sigma)} y_{j}^{M_{j}(\sigma)-1} d y_{j} .
\end{aligned}
$$

Let us give the other formulae of $G_{ \pm}(f, g, \varphi)$, which are more directly expressed by $f, g, \varphi$. From Lemma 7.1 with Remark 11.4, we obtain

$$
\begin{aligned}
& \left(f_{\tau_{*}(\sigma)} \circ \pi(\sigma)\right)\left(T_{A(\sigma)}^{1}(y)\right)=\left(\prod_{j \notin A(\sigma)} y_{j}^{l_{f}\left(a^{j}(\sigma)\right)}\right) f_{\sigma}\left(T_{A(\sigma)}(y)\right), \\
& \left(g_{\gamma_{*}(\sigma)} \circ \pi(\sigma)\right)\left(T_{A(\sigma)}^{1}(y)\right)=\left(\prod_{j \notin A(\sigma)} y_{j}^{l_{g}\left(a^{j}(\sigma)\right)}\right) g_{\sigma}\left(T_{A(\sigma)}(y)\right) .
\end{aligned}
$$

By using the above equations, (11.19) can be rewritten as

$$
\begin{aligned}
& G_{ \pm}(f, g, \varphi) \\
& =L_{\sigma} \int_{\mathbb{R}_{+}^{n-m(f, g)}} \frac{\left(g_{\gamma_{*}(\sigma)} \circ \pi(\sigma)\right)\left(T_{A(\sigma)}^{1}(y)\right)(\varphi \circ \pi(\sigma))\left(T_{A(\sigma)}(y)\right)}{\left(\left(f_{\tau_{*}(\sigma)} \circ \pi(\sigma)\right)\left(T_{A(\sigma)}^{1}(y)\right)\right)_{ \pm}^{1 / d(f, g)}} \prod_{j \notin A(\sigma)} y_{j}^{\left\langle a^{j}(\sigma)\right\rangle-1} d y_{j} .
\end{aligned}
$$

We remark that $G_{ \pm}(f, g, \varphi)$ depend only on $f_{\tau_{*}(\sigma)}, g_{\gamma_{*}(\sigma)}, \varphi$ and that $G_{ \pm}(f, g, \varphi)$ must be independent of the selection of $\sigma \in \Sigma_{*}^{(n)}$.

Under the hypothesis (b). In a similar fashion to the proof of Proposition 10.6. we can show the assertion in the case when the hypothesis (b) is satisfied.

The case: $m(f, g)=n$.

In this case, we see that $A(\sigma)=\{1, \ldots, n\}$. We remark that $\tau_{*}(\sigma)$ and $\gamma_{*}(\sigma)$ are vertices of $\Gamma_{+}(f)$ and $\Gamma_{+}(g)$, denoted by $q$ and $p$ respectively. Similar computations give the following expression corresponding to (11.19):

$$
G_{ \pm}(f, g, \varphi)=L \frac{g_{\sigma}(0) \varphi(0)}{f_{\sigma}(0)_{ \pm}^{1 / d(f, g)}}
$$

where $\sigma \in \Sigma_{*}^{(n)}$ and $L=\sum_{\sigma \in \Sigma_{*}^{(n)}} L_{\sigma}$. From the equation (11.20), we obtain another expression corresponding to (11.21):

$$
G_{ \pm}(f, g, \varphi)=L \frac{g_{\gamma_{*}(\sigma)}(\mathbf{1}) \varphi(0)}{f_{\tau_{*}(\sigma)}(\mathbf{1})_{ \pm}^{1 / d(f, g)}}=L \frac{(q !)^{1 / d(f, g)}}{p !} \frac{\left(\partial^{p} g\right)(0) \varphi(0)}{\left(\partial^{q} f\right)(0)_{ \pm}^{1 / d(f, g)}}
$$

where $\mathbf{1}=(1, \ldots, 1)$ and $L$ is the same as in (11.22).

Remark 11.10. Let us consider the case when $\tau_{*}(\sigma)$ or $\gamma_{*}(\sigma)$ is compact. Note that the compactness of $\tau_{*}(\sigma)$ is equivalent to that of $\gamma_{*}(\sigma)$ (see Remark 2.10). Lemma 6.4 with Remark 11.4 implies that $\pi(\sigma)\left(T_{A(\sigma)}\left(\mathbb{R}^{n}\right)\right)=0$. Therefore, the 
formulae (11.19), (11.21) can be expressed in simpler forms as follows:

$$
\begin{aligned}
& G_{ \pm}(f, g, \varphi)=L_{\sigma} \varphi(0) \int_{\mathbb{R}_{+}^{n-m(f, g)}} \frac{g_{\sigma}\left(T_{A(\sigma)}(y)\right)}{f_{\sigma}\left(T_{A(\sigma)}(y)\right)_{ \pm}^{1 / d(f, g)}} \prod_{j \notin A(\sigma)} y_{j}^{M_{j}(\sigma)-1} d y_{j} \\
& =L_{\sigma} \varphi(0) \int_{\mathbb{R}_{+}^{n-m(f, g)}} \frac{\left(g_{\gamma_{*}(\sigma)} \circ \pi(\sigma)\right)\left(T_{A(\sigma)}^{1}(y)\right)}{\left(\left(f_{\tau_{*}(\sigma)} \circ \pi(\sigma)\right)\left(T_{A(\sigma)}^{1}(y)\right)\right)_{ \pm}^{1 / d(f, g)}} \prod_{j \notin A(\sigma)} y_{j}^{\left\langle a^{j}(\sigma)\right\rangle-1} d y_{j} .
\end{aligned}
$$

Finally, let us compute the coefficients of $(s+1 / d(f, g))^{-m(f, g)}$ in the Laurent expansions of $Z_{ \pm}(s ; \varphi), Z(s ; \varphi)$. Respectively, we define

$$
\begin{aligned}
& C_{ \pm}=\lim _{s \rightarrow-1 / d(f, g)}(s+1 / d(f, g))^{m(f, g)} Z_{ \pm}(s ; \varphi), \\
& C=\lim _{s \rightarrow-1 / d(f, g)}(s+1 / d(f, g))^{m(f, g)} Z(s ; \varphi) .
\end{aligned}
$$

Theorem 11.11. Suppose that (i) $f$ satisfies the condition (E), (ii) $g$ belongs to the class $\hat{\mathcal{E}}(U)$ and (iii) at least one of the following conditions is satisfied:

(a) $d(f, g)>1$.

(b) $f$ is nonnegative or nonpositive on $U$.

(c) There exists a principal face $\tau_{*}$ of $\Gamma_{+}(f)$ such that $f_{\tau_{*}}$ does not vanish on $U \cap(\mathbb{R} \backslash\{0\})^{n}$.

Then we obtain explicit formulae for coefficients in the following:

$$
C_{ \pm}=\sum_{\theta \in\{-1,1\}^{n}} G_{ \pm}\left(f_{\theta}, g_{\theta}, \varphi_{\theta}\right) \quad \text { and } \quad C=C_{+}+C_{-}
$$

where $f_{\theta}(x):=f\left(\theta_{1} x_{1}, \ldots, \theta_{n} x_{n}\right)$, etc., and $G_{ \pm}(f, g, \varphi)$ are as in (11.19), (11.21), (11.22), (11.23) in the proof of Proposition 11.9.

Furthermore, the formulae (11.25) imply the following. Suppose that (iv) $g_{\gamma_{*}}$ is nonnegative (resp. nonpositive) on $U$, where $\gamma_{*}$ is a principal face of $\Gamma_{+}(g)$ (associated to $\tau_{*}$ if (c) is satisfied in (iii)), and $(\mathrm{v}) \varphi(0)>0$ and $\varphi$ is nonnegative on $U$. Then, if the support of $\varphi$ is sufficiently small, then $C_{ \pm}$are nonnegative (resp. nonpositive) and $C=C_{+}+C_{-}$is positive (resp. negative).

Proof. Remembering how to show Theorem 10.8 by using Proposition 10.6, one can almost similarly show the above theorem by using Proposition 11.9, The following show nontrivial parts.

- The surjectivities in Lemma 11.8 are used to find a cone associated to principal faces which satisfy the conditions in the theorem.

- In order to see $C=C_{+}+C_{-} \neq 0$ from the formula (11.21), it must be shown that $g_{\gamma_{*}} \circ \pi(\sigma) \circ T_{A(\sigma)}^{1}$ does not identically equal zero near the origin. Indeed, this follows from the equations (11.20) with $g_{\sigma}(0) \neq 0$.

11.4. Poles on negative integers. Let us consider the poles of $Z_{ \pm}(s ; \varphi)$ on negative integers. For $\lambda \in-\mathbb{N}$, define

$$
\begin{aligned}
& A_{\lambda}(\sigma):=\left\{j \in B(\sigma): l_{f}\left(a^{j}(\sigma)\right) \lambda+l_{g}\left(a^{j}(\sigma)\right)+\left\langle a^{j}(\sigma)\right\rangle-1 \in-\mathbb{N}\right\}, \\
& \rho_{\lambda}:=\min \left\{\max \left\{\# A_{\lambda}(\sigma): \sigma \in \Sigma^{(n)}\right\}, n-1\right\} .
\end{aligned}
$$

We obtain the following result corresponding to Proposition 10.9. 
Proposition 11.12. Suppose that (i) $f$ satisfies the condition (E) and that (ii) $g$ belongs to the class $\hat{\mathcal{E}}(U)$. If the support of $\varphi$ is contained in a sufficiently small neighborhood of the origin, then the orders of poles of $Z_{ \pm}(s ; \varphi)$ at $s=\lambda \in-\mathbb{N}$ are not higher than $\rho_{\lambda}+1$. In particular, if $\lambda>-1 / d(f, g)$, then these orders are not higher than 1. Moreover, let $a_{\lambda}^{ \pm}$be the coefficients of $(s-\lambda)^{-\rho_{\lambda}-1}$ in the Laurent expansions of $Z_{ \pm}(s ; \varphi)$ at $s=\lambda$, respectively; then we have $a_{\lambda}^{+}=(-1)^{\lambda-1} a_{\lambda}^{-}$for $\lambda \in-\mathbb{N}$.

Proof. The difference between (10.7) and (11.4) does not essentially affect the argument in the proof of Proposition 10.9. The details are left to the reader.

\section{The CASE OF CONVEnient $f$}

Let us consider the poles of $Z_{ \pm}(s ; \varphi)$ and $Z(s ; \varphi)$ in the case when the function $f$ is convenient. In this case, all the results in Section 11 can be obtained without the hypothesis $g \in \hat{\mathcal{E}}(U)$.

In this section, we only assume that $g$ is a nonflat smooth function defined on an open neighborhood $U$ of the origin and we use the same notation as in Section 11 . The purpose of this section is to show the following theorem.

Theorem 12.1. In Theorems 11.1, 11.11 and Propositions 11.9, 11.12, replacing the condition (ii) " $g$ belongs to the class $\hat{\mathcal{E}}(U)$ " by the condition " $f$ is convenient", we have the same assertions.

After the above exchange in Theorem 11.1, the new theorem will be expressed by Theorem 11.1'. The other new theorem and propositions will be expressed in a similar fashion.

12.1. Why the case of convenient $f$ can be conveniently treated. Before exactly proving Theorem 12.1. we roughly explain the essential reason why the convenience of $f$ can give the assertions without strong hypotheses of $g$. Hereafter, we assume that $\varphi$ is always nonnegative on $U$ and $\varphi(0)>0$.

First, let us recall and consider geometrical relationship between the Newton distance $d(f, g)$ and the two Newton polyhedra $\Gamma_{+}(f)$ and $\Gamma_{+}(g)$. When $d>0$ is large enough, the two sets $(1 / d) \cdot \partial \Gamma_{+}(f)$ and $\Gamma_{+}(g)+\mathbf{1}$ are separate, where $\partial \Gamma_{+}(f)$ is the topological boundary of $\Gamma_{+}(f)$ and $\mathbf{1}=(1, \ldots, 1)$. As $d$ tends to zero, the set $(1 / d) \cdot \partial \Gamma_{+}(f)$ gradually moves towards the set $\Gamma_{+}(g)+\mathbf{1}$ and, finally, $(1 / d) \cdot \partial \Gamma_{+}(f)$ contacts $\Gamma_{+}(g)+\mathbf{1}$ from the outside. The value of this $d$ defines the Newton distance $d(f, g)$. Theorem 11.11 asserts that

$$
\text { "the leading pole of } Z(s ; \varphi) \text { exists at } s=-1 / d(f, g) "
$$

under some appropriate conditions.

In order to understand the validity of the convenience condition of $f$ for the assertion (12.1), let us observe a typical problem occurring in the case when $f$ is not convenient and $g$ does not belong to $\hat{\mathcal{E}}(U)$. Consider the following two-dimensional example:

$$
f\left(x_{1}, x_{2}\right)=x_{1}^{4} ; \quad g\left(x_{1}, x_{2}\right)=x_{1}^{2} x_{2}^{2}+e^{-1 / x_{2}^{2}} .
$$


This example has appeared in Remark 3.4 and it is a special case of Example 1 in Section 15 (consider the case when $c=1, p=1, q=0$ ). The Newton data about these $f$ and $g$ can be easily seen, and we have

$$
\begin{aligned}
& (1 / d) \cdot \Gamma_{+}(f)=\left\{\left(\alpha_{1}, \alpha_{2}\right) \in \mathbb{R}_{+}^{2}: \alpha_{1} \geq 4 / d\right\}, \\
& \Gamma_{+}(g)+\mathbf{1}=\left\{\left(\alpha_{1}, \alpha_{2}\right) \in \mathbb{R}_{+}^{2}: \alpha_{1} \geq 3, \alpha_{2} \geq 3\right\}, \\
& d(f, g)=4 / 3 .
\end{aligned}
$$

Of course, the flat function term $e^{-1 / x_{2}^{2}}$ in $g$ does not give any geometrical information of the Newton polyhedron of $g$. However, this term analytically affects the properties of $Z(s ; \varphi)$ and it actually gives an obstruction for the assertion (12.1). In fact, the leading pole of $Z(s ; \varphi)$ exists at $s=-1 / 4$, which does not equal $-1 / d(f, g)=-3 / 4$.

Let us try to forcibly give geometrical Newton data induced by the term $e^{-1 / x_{2}^{2}}$ and to understand how the leading pole of $Z(s ; \varphi)$ appears at $s=-1 / 4$. Since $e^{-1 / x_{2}^{2}}=O\left(x_{2}^{N}\right)$ for any positive integer $N$, it might be interpreted that there exists something like a speck of dust $\{(0, N)\}$ very far from the origin outside the Newton polyhedron of $g$ and, more exactly, the set $\Gamma_{+}(g)+\mathbf{1}$ should be approximated as follows:

$$
\Gamma_{+}(g)+\mathbf{1} \approx \text { convex hull of }\left(\left\{\left(\alpha_{1}, \alpha_{2}\right) \in \mathbb{R}_{+}^{2}: \alpha_{1} \geq 3, \alpha_{2} \geq 3\right\} \cup\{(1, N+1)\}\right),
$$

with $N \gg 1$. This approximated Newton polyhedron plays a more essential role in the analysis of poles of $Z(s ; \varphi)$. Indeed, let us replace the real Newton polyhedron of $g$ by the above approximated one and let $d$ tend to zero. Before contacting with the real polyhedron $\Gamma_{+}(g)+\mathbf{1}$, the set $(1 / d) \cdot \partial \Gamma_{+}(f)$ intersects the approximated one on the vertical edge containing the point $\{(1, N+1)\}$. The value of this $d$ is 4 , which determines the position of the leading pole of $Z(s ; \varphi)$ from the computation in Section 15.1. It easily follows from this observation that these kinds of problems can occur only when $\Gamma_{+}(f)$ does not intersect some coordinate hyperplane. In order to refuse these problems when $f$ is not convenient, we need some kind of assumption on $g$ for the assertion (12.1), which reveals that there is completely nothing outside the set $(1 / d(f, g)) \cdot \Gamma_{+}(f)$. For example, the inclusion $\Gamma(g)+\mathbf{1} \subset(1 / d(f, g)) \cdot \partial \Gamma_{+}(f)$ implies that the condition $g \in \hat{\mathcal{E}}(U)$ is sufficient for this necessary assumption.

In the case when $f$ is convenient, fortunately, these kinds of problems cannot occur. Indeed, if $f$ is convenient, then the complement of the set $(1 / d) \cdot \Gamma_{+}(f)$ in $\mathbb{R}_{+}^{2}$ is bounded. Even if a speck of dust induced from the flat function like the above $\{(0, N)\}$ exists, then since it is always very far from the origin, the set $(1 / d) \cdot \partial \Gamma_{+}(f)$ does not intersect it before contacting with $\Gamma_{+}(g)+\mathbf{1}$. This is an essential reason why the case when $f$ is convenient can be easily treated.

12.2. Proof of Theorem 12.1. Before proving the above theorem, we prepare two lemmas. For $a \in \mathbb{Z}_{+}^{n}$, we define

$$
\tilde{l}_{g}(a)= \begin{cases}l_{g}(a) & \text { for } a \in \mathbb{N}^{n}, \\ 0 & \text { for } a \in \mathbb{Z}_{+}^{n} \backslash \mathbb{N}^{n} .\end{cases}
$$


Lemma 12.2. Suppose that $\sigma \in \Sigma^{(n)}$. Then we have the following.

(i) There exists a smooth function $\tilde{g}_{\sigma}$ defined on $\pi(\sigma)^{-1}(U)$ such that

$$
g(\pi(\sigma)(y))=\left(\prod_{j=1}^{n} y_{j}^{\tilde{l}_{g}\left(a^{j}(\sigma)\right)}\right) \tilde{g}_{\sigma}(y) \quad \text { for } y \in \pi(\sigma)^{-1}(U) .
$$

We remark that $\tilde{g}_{\sigma}$ may vanish at the origin.

(ii) Let $\gamma$ be a compact face of $\Gamma_{+}(g)$ defined by

$$
\gamma:=\bigcap_{j \in I} H\left(a^{j}(\sigma), l_{g}\left(a^{j}(\sigma)\right)\right) \cap \Gamma_{+}(g) \quad \text { with } I \subset\left\{j: a_{j}(\sigma) \in \mathbb{N}^{n}\right\} .
$$

(In terms of symbols in Section [6.2, $\gamma=\gamma(I, \sigma)$ with $P=\Gamma_{+}(g)$.) Then, we have

$$
g_{\gamma}(\pi(\sigma)(y))=\left(\prod_{j=1}^{n} y_{j}^{\tilde{l}_{g}\left(a^{j}(\sigma)\right)}\right) \tilde{g}_{\sigma}\left(T_{I}(y)\right) \quad \text { for } y \in \pi(\sigma)^{-1}(U) .
$$

(iii) Let $I$ be as in (ii) and let $g_{\sigma}$ be defined by

$$
g_{\sigma}(y)=\left(\prod_{j \notin I} y_{j}^{\tilde{l}_{g}\left(a^{j}(\sigma)\right)-l_{g}\left(a^{j}(\sigma)\right)}\right) \tilde{g}_{\sigma}\left(T_{I}(y)\right) \quad \text { for } y \in \pi(\sigma)^{-1}(U) .
$$

Then $g_{\sigma}$ is a polynomial of only variables $y_{j}$ with $j \notin I$ and $g_{\sigma}(0) \neq 0$. Note that $g_{\sigma}\left(T_{I}(y)\right)=g_{\sigma}(y)$.

(iv) Let $\gamma, I$ be as in (ii). Then we have

$$
g_{\gamma}\left(\pi(\sigma)\left(T_{I}^{1}(y)\right)\right)=\left(\prod_{j \notin I} y_{j}^{l_{g}\left(a^{j}(\sigma)\right)}\right) g_{\sigma}\left(T_{I}(y)\right) \quad \text { for } y \in \pi(\sigma)^{-1}(U) .
$$

Remark 12.3. When $g$ does not belong to $\hat{\mathcal{E}}(U)$, the smooth function $g_{\sigma}$ cannot always be defined by applying Lemma 7.1, When we prove Theorem 12.1, we use the function $g_{\sigma}$, which is a polynomial defined as in (iii) in the above lemma.

Proof of Lemma 12.2. (i) Let $\Sigma_{+}^{(1)}$ be the subset of $\Sigma^{(1)}$ defined by $\Sigma_{+}^{(1)}:=\{a \in$ $\left.\Sigma^{(1)}: a \in \mathbb{N}^{n}\right\}$. We remark that the convenience condition of $f$ implies that $\Sigma_{+}^{(1)}$ is not an empty set. Let

$$
\tilde{\Gamma}_{+}(g):=\bigcap_{a \in \Sigma_{+}^{(1)}} H^{+}\left(a, l_{g}(a)\right) \cap \mathbb{R}_{+}^{n} .
$$

Then, $\tilde{\Gamma}_{+}(g)$ is a polyhedron containing $\Gamma_{+}(g)$. Note that $g$ is convenient if and only if $\Gamma_{+}(g)=\tilde{\Gamma}_{+}(g)$. It follows from Definition 2.16 that $g$ belongs to $\hat{\mathcal{E}}\left[\tilde{\Gamma}_{+}(g)\right](U)$. From Proposition 2.17, we can obtain the expression of the form (2.14) with $f=g$ and $P=\tilde{\Gamma}_{+}(g)$. Furthermore, substituting $x=\pi(\sigma)(y)$ in (5.1) into this expression, we obtain

$$
g(\pi(\sigma)(y))=\sum_{p \in S}\left(\prod_{j=1}^{n} y_{j}^{\left\langle a^{j}(\sigma), p\right\rangle}\right) \psi_{p}(\pi(\sigma)(y)) .
$$


Now, let us set

$$
\tilde{g}_{\sigma}(y):=\sum_{p \in S}\left(\prod_{j=1}^{n} y_{j}^{\left\langle a^{j}(\sigma), p\right\rangle-\tilde{l}_{g}\left(a^{j}(\sigma)\right)}\right) \psi_{p}(\pi(\sigma)(y)) .
$$

Then $\tilde{g}_{\sigma}$ is a smooth function defined on the set $\pi(\sigma)^{-1}(U)$ and satisfies (12.4).

(ii) Let $\tilde{\gamma}$ be a face of $\tilde{\Gamma}_{+}(g)$ defined by $\tilde{\gamma}:=\bigcap_{j \in I} H\left(a^{j}(\sigma), l_{g}\left(a^{j}(\sigma)\right)\right) \cap \tilde{\Gamma}_{+}(g)$. In terms of symbols in Section 6.2 this equation is written as $\tilde{\gamma}=\gamma(I, \sigma)$ with $P=\tilde{\Gamma}_{+}(g)$. Thus, the equation (7.4) in Lemma 7.1 implies that $g_{\tilde{\gamma}}(\pi(\sigma)(y))$ equals the right hand side of (12.5). The compactness of $\gamma$ implies $g_{\tilde{\gamma}}=g_{\gamma}$. So, we obtain (12.5).

(iii) From Proposition 2.17 and the compactness of $\gamma, g_{\gamma}$ can be written as $g_{\gamma}(x)=\sum_{p \in \gamma \cap \mathbb{Z}_{+}^{n}} x^{p} \psi_{p}(0)$, where $\psi_{p}(0) \neq 0$ if $p$ is a vertex of $\gamma$. Substituting $x=\pi(\sigma)(y)$ with (5.1), we have

$$
g_{\gamma}(\pi(\sigma)(y))=\sum_{p \in \gamma \cap \mathbb{Z}_{+}^{n}}\left(\prod_{j=1}^{n} y_{j}^{\left\langle a^{j}(\sigma), p\right\rangle}\right) \psi_{p}(0) .
$$

Putting (12.5), (12.8) together, we have

$$
\tilde{g}_{\sigma}\left(T_{I}(y)\right)=\sum_{p \in \gamma \cap \mathbb{Z}_{+}^{n}}\left(\prod_{j \notin I} y_{j}^{\left\langle a^{j}(\sigma), p\right\rangle-\tilde{l}_{g}\left(a^{j}(\sigma)\right)}\right) \psi_{p}(0) .
$$

Moreover, putting (12.6), (12.9) together, we have

$$
g_{\sigma}(y)=\sum_{p \in \gamma \cap \mathbb{Z}_{+}^{n}}\left(\prod_{j \notin I} y_{j}^{\left\langle a^{j}(\sigma), p\right\rangle-l_{g}\left(a^{j}(\sigma)\right)}\right) \psi_{p}(0) .
$$

It directly follows from the above equation that $g_{\sigma}$ is a polynomial of $y_{j}$ with $j \notin I$ and that $g_{\sigma}(0)=\psi_{p}(0)(\neq 0)$ where $p$ is some vertex of $\Gamma_{+}(g)$.

(iv) From (12.5), (12.6), we obtain (12.7).

Lemma 12.4. Let $a$ be in $\mathbb{Z}_{+}^{n}$. If $f$ is convenient and $l_{f}(a) \neq 0$, then $a \in \mathbb{N}^{n}$.

Proof. Noticing $l_{f}(a)=0$ when $f$ is convenient and $a \in \mathbb{Z}_{+}^{n} \backslash \mathbb{N}^{n}$, we can obtain the lemma.

Proof of Theorem 11.1'. Instead of (11.2), the equation (12.4) is substituted into (11.3). Then integrals analogous to (11.4) can be obtained. From these integrals, we similarly see the set of candidate poles as follows:

$$
\left\{-\frac{\tilde{l}_{g}\left(a^{j}(\sigma)\right)+\left\langle a^{j}(\sigma)\right\rangle+\nu}{l_{f}\left(a^{j}(\sigma)\right)}: \nu \in \mathbb{Z}_{+}, j \in B(\sigma)\right\} \cup(-\mathbb{N}) .
$$

Notice that $j \in B(\sigma) \Longleftrightarrow l_{f}\left(a^{j}(\sigma)\right) \neq 0 \Longrightarrow a^{j}(\sigma) \in \mathbb{N}^{n}$ from Lemma 12.4, which implies $\tilde{l}_{g}\left(a^{j}(\sigma)\right)=l_{g}\left(a^{j}(\sigma)\right)$. Therefore, we see that the set (12.10) is contained in the set (11.1). The exchange in the assumption does not affect the rest of the assertions in Theorem 11.1 . 
Proof of Proposition 11.9'. We show only the case when $m(f, g)<n$ and the hypothesis (a), because the other cases can be easily shown by using the proof of Proposition 11.9.

Let us compute the limits $\tilde{C}_{ \pm}$in (11.16). From the integrals in the proof of Theorem $11.1^{\prime}$, which are analogous to (11.4), we can see that the limits $\tilde{C}_{ \pm}$take the values analogous to $G_{ \pm}(f, g, \varphi)$ in (11.19). Indeed, these values are obtained by exchanging $g_{\sigma}, l_{g}\left(a^{j}(\sigma)\right)$ with $\tilde{g}_{\sigma}, \tilde{l}_{g}\left(a^{j}(\sigma)\right)$ in (11.19), respectively. Substituting the first equation in (11.20) and the equation in (12.5) with $\gamma=\gamma_{*}(\sigma)$ and $I=A(\sigma)$, we obtain the equations (11.21). We remark that at present the nonvanishing of $G_{+}+G_{-}$cannot been shown, because $\tilde{g}_{\sigma}(0)$ may vanish.

Furthermore, substituting the first equation in (11.20) and the equation in (12.5) with $\gamma=\gamma_{*}(\sigma)$ and $I=A(\sigma)$ into (11.21), we obtain (11.19).

Proof of Theorem 11.11'. In a similar fashion to the proof of Theorem 11.11, we can obtain Theorem $11.11^{\prime}$ by using Proposition $11.9^{\prime}$. Note that the nonvanishing of $C=C_{+}+C_{-}$can be similarly seen from the fact that $g_{\sigma}(0) \neq 0$.

Proof of Proposition 11.12'. This can be similarly shown by using the integrals analogous to (11.4), which were obtained in the proof of Theorem 11.1'.

\section{Certain symmetry Properties}

We denote by $\beta_{ \pm}(f, g), \hat{\beta}(f, g)$ the largest poles of $Z_{ \pm}(s ; \varphi), Z(s ; \varphi)$ and by $\eta_{ \pm}(f, g), \hat{\eta}(f, g)$ their orders, respectively. In this section, we give some symmetry properties of $\beta_{ \pm}(f, g), \hat{\beta}(f, g), \eta_{ \pm}(f, g), \hat{\eta}(f, g)$.

Theorem 13.1. Suppose that $f, g$ satisfy the condition (E) and are nonnegative or nonpositive on $U$. If the support of $\varphi$ is contained in a sufficiently small neighborhood of the origin, then we have

$$
\beta_{ \pm}\left(x^{\mathbf{1}} f, g\right) \beta_{ \pm}\left(x^{\mathbf{1}} g, f\right) \leq 1 \text { and } \hat{\beta}\left(x^{\mathbf{1}} f, g\right) \hat{\beta}\left(x^{\mathbf{1}} g, f\right) \leq 1 .
$$

Moreover, the following two conditions are equivalent:

(i) The equality holds in each estimate in (13.1).

(ii) There exists a positive rational number $d$ such that $\Gamma_{+}\left(x^{\mathbf{1}} f\right)=d \cdot \Gamma_{+}\left(x^{\mathbf{1}} g\right)$. If the condition (i) or (ii) is satisfied, then we have $\eta_{ \pm}\left(x^{\mathbf{1}} f, g\right)=\eta_{ \pm}\left(x^{\mathbf{1}} g, f\right)=$ $\hat{\eta}\left(x^{\mathbf{1}} f, g\right)=\hat{\eta}\left(x^{\mathbf{1}} g, f\right)=n$.

Proof. We only consider the case of $Z_{ \pm}(s ; \varphi)$.

Admitting Lemmas 13.2 and 13.3 below, we can prove the theorem as follows. From the assumptions in the theorem and Lemma 13.2. Theorem 11.11 implies the equations $\beta\left(x^{\mathbf{1}} f, g\right)=-1 / d\left(x^{\mathbf{1}} f, g\right)$ and $\beta\left(x^{\mathbf{1}} g, f\right)=-1 / d\left(x^{\mathbf{1}} g, f\right)$. By Lemma 13.3, these equations imply the inequalities (13.1) and the equivalence of (i) and (ii). The condition (ii) in the theorem implies that $d \cdot\left(\Gamma_{+}(g)+\mathbf{1}\right)=\Gamma_{+}\left(x^{\mathbf{1}} f\right)$ with $d=d\left(x^{\mathbf{1}} f, g\right)$ and, moreover, that $\Gamma_{0}\left(x^{\mathbf{1}} f\right)=\partial \Gamma_{+}\left(x^{\mathbf{1}} f\right)$ for the pair $\left(x^{\mathbf{1}} f, g\right)$. Thus, each principal face of $\Gamma_{+}(g)$ is a vertex (i.e., zero-dimensional face) of $\Gamma_{+}(g)$. (See Remark 2.7.) This means $\eta_{ \pm}\left(x^{\mathbf{1}} f, g\right)=m\left(x^{\mathbf{1}} f, g\right)=n$. Similarly, we see that $\eta_{ \pm}\left(x^{\mathbf{1}} g, f\right)=n$.

Lemma 13.2. Let $f$ be a smooth nonflat function defined on a neighborhood of the origin in $\mathbb{R}^{n}$. Then $f$ is nondegenerate over $\mathbb{R}$ with respect to its Newton polyhedron if and only if so is $x^{\mathbf{1}} f$. 
Proof. This is a special case of Lemma 7.8.

Lemma 13.3. Let $f, g$ be smooth functions defined near the origin. Then we have

$$
d\left(x^{\mathbf{1}} f, g\right) d\left(x^{\mathbf{1}} g, f\right) \geq 1 .
$$

The equality holds in the above if and only if there exists a positive rational number $d$ such that $\Gamma_{+}\left(x^{\mathbf{1}} f\right)=d \cdot \Gamma_{+}\left(x^{\mathbf{1}} g\right)$.

Proof. See Lemma 5.19 in $[5]$.

\section{Proofs of the theorems in Section 4}

14.1. Relationship between $I(t ; \varphi)$ and $Z_{ \pm}(s ; \varphi)$. It is known (see [18, [1, etc.) that the study of the asymptotic behavior of the oscillatory integral $I(t ; \varphi)$ in (1.1) can be reduced to an investigation of the poles of the functions $Z_{ \pm}(s ; \varphi)$ in (8.1). Let us explain an outline of this reduction. Let $f$ and $\varphi$ satisfy the conditions (A) and (C) in Section 3 respectively and let $g$ be a smooth function on an open neighborhood of the origin in $\mathbb{R}^{n}$ ( $g$ is possibly flat). Suppose that the support of $\varphi$ is sufficiently small.

Define the weighted Gelfand-Leray function: $K: \mathbb{R} \rightarrow \mathbb{R}$ as

$$
K(c)=\int_{W_{c}} g(x) \varphi(x) \omega,
$$

where $W_{c}=\left\{x \in \mathbb{R}^{n}: f(x)=c\right\}$ and $\omega$ is the surface element on $W_{c}$ which is determined by $d f \wedge \omega=d x_{1} \wedge \cdots \wedge d x_{n} . I(t ; \varphi)$ and $Z_{ \pm}(s ; \varphi)$ can be expressed by using $K(c)$ : Changing the integral variables in (1.1), (8.1), we have

$$
\begin{gathered}
I(t ; \varphi)=\int_{-\infty}^{\infty} e^{i t c} K(c) d c=\int_{0}^{\infty} e^{i t c} K(c) d c+\int_{0}^{\infty} e^{-i t c} K(-c) d c, \\
Z_{ \pm}(s ; \varphi)=\int_{0}^{\infty} c^{s} K( \pm c) d c
\end{gathered}
$$

respectively. Applying the inverse formula of the Mellin transform to (14.3), we have

$$
K( \pm c)=\frac{1}{2 \pi i} \int_{r-i \infty}^{r+i \infty} Z_{ \pm}(s ; \varphi) c^{-s-1} d s
$$

where $r>0$ and the integral contour follows the line $\operatorname{Re}(s)=r$ upwards. Recall that $Z_{+}(s ; \varphi)$ and $Z_{-}(s ; \varphi)$ are meromorphic functions and their poles exist on the negative part of the real axis. By deforming the integral contour as $r$ tends to $-\infty$ in (14.4), the residue formula gives the asymptotic expansions of $K(c)$ as $c \rightarrow \pm 0$. Substituting these expansions of $K(c)$ into (14.2), we can get an asymptotic expansion of $I(t ; \varphi)$ as $t \rightarrow+\infty$.

Through the above calculation, we see a more precise relationship for the coefficients. If $Z_{+}(s ; \varphi)$ and $Z_{-}(s ; \varphi)$ have the Laurent expansions at $s=-\lambda$,

$$
Z_{ \pm}(s ; \varphi)=\frac{B_{ \pm}}{(s+\lambda)^{\rho}}+O\left(\frac{1}{(s+\lambda)^{\rho-1}}\right)
$$

respectively, then the corresponding part in the asymptotic expansion of $I(t ; \varphi)$ has the form

$$
B t^{-\lambda}(\log t)^{\rho-1}+O\left(t^{-\lambda}(\log t)^{\rho-2}\right) .
$$


Here a simple computation gives the following relationship:

$$
B=\frac{\Gamma(\lambda)}{(\rho-1) !}\left[e^{i \pi \lambda / 2} B_{+}+e^{-i \pi \lambda / 2} B_{-}\right]
$$

where $\Gamma$ is the Gamma function.

Remark 14.1. If $\lambda$ is not an odd integer, then

$$
\operatorname{Re}(B)=\frac{2 \Gamma(\lambda) \cos (\pi \lambda / 2)}{(\rho-1) !}\left(B_{+}+B_{-}\right)
$$

In order to decide the vanishing of the coefficient, the above equation is helpful.

14.2. The first coefficient in the asymptotics of $I(t ; \varphi)$. From the relationship between $I(t ; \varphi)$ and $Z_{ \pm}(s ; \varphi)$ in the previous section and the equation (14.5), we give explicit formulae for the coefficient of the leading term of the asymptotic expansion (1.2) of $I(t ; \varphi)$ at infinity as follows.

Theorem 14.2. If $f$ and $g$ satisfy the conditions (i), (ii), (iv) in Theorem 4.4 and the support of $\varphi$ is contained in a sufficiently small neighborhood of the origin, then we have

$$
\begin{aligned}
\lim _{t \rightarrow \infty} & t^{1 / d(f, g)}(\log t)^{-m(f, g)+1} \cdot I(t ; \varphi) \\
& =\frac{\Gamma(1 / d(f, g))}{(m(f, g)-1) !}\left[e^{i \pi /(2 d(f, g))} C_{+}+e^{-i \pi /(2 d(f, g))} C_{-}\right],
\end{aligned}
$$

where $C_{ \pm}$are as in (11.25) in Theorem 11.11.

Remark 14.3. In the unweighted case, analogous results have been obtained in [27, [7, [10.

14.3. Proofs of Theorems 4.1, 4.4 and 4.7. Applying the argument in Section 14.1 to the results relating to $Z_{ \pm}(s ; \varphi)$ in Sections 11 and 12, we obtain the theorems in Section 4 .

Proof of Theorem 4.1. This theorem follows from Theorems 11.1 and 12.1. Notice that Proposition 11.12 and the relationship (14.5) induce the cancellation of the coefficients of the term whose decay rates are larger than $t^{-1 / d(f, g)}(\log t)^{m(f, g)-1}$.

Proof of the estimate (4.2) in Remark 4.3. This estimate follows from Theorem 10.1 by using the relationship in Section 14.1. One must consider the cancellation similar to that in the proof of Theorem 4.1

Proof of Theorem 4.4. This theorem follows from Theorem 14.2 by considering the assertions in Theorems 11.11 and 12.1. Note that the necessity of the condition in (iii-c), " $-1 / d(f, g)$ is not an odd integer", follows from Remark 14.1. 
14.4. Generalization of Varchenko's results to the Puiseux series case. By using the results about local zeta type functions with respect to the class $C_{1 / p}^{\infty}\left(U_{+}\right)$ introduced in Section 10.4, we can give some kind of generalization of the results about unweighted oscillatory integrals due to Varchenko [30] and Kamimoto and Nose [20].

Let $U$ be an open neighborhood of the origin in $\mathbb{R}^{n}$ and let $p:=\left(p_{1}, \ldots, p_{n}\right) \in$ $\mathbb{N}^{n}$. The definitions of the function spaces $C_{1 / p}^{\infty}\left(U_{+}\right)$and $\hat{\mathcal{E}}_{1 / p}\left(U_{+}\right)$were given in Section 10.4. Recall that every element of $C_{1 / p}^{\infty}\left(U_{+}\right)$or $\hat{\mathcal{E}}_{1 / p}\left(U_{+}\right)$can be considered to admit the following fractional power series at the origin:

$$
f(x) \sim \sum_{\alpha \in \mathbb{Z}_{+}^{n}} c_{\alpha} x^{\alpha / p}:=\sum_{\alpha \in \mathbb{Z}_{+}^{n}} c_{\alpha} x_{1}^{\alpha_{1} / p_{1}} \cdots x_{n}^{\alpha_{n} / p_{n}} .
$$

For $f \in C_{1 / p}^{\infty}\left(U_{+}\right)$, we define

$$
\tilde{I}(t ; \varphi):=\int_{\mathbb{R}_{+}^{n}} e^{i t f(x)} \varphi(x) d x,
$$

where $t>0$ and $\varphi: U \rightarrow \mathbb{R}$ satisfies the condition (C) in Section 3 .

The assertions of the following theorem are almost the same as those of Theorems 3.7 and 3.1 in appearance.

Theorem 14.4. Suppose that $f$ belongs to the class $\hat{\mathcal{E}}_{1 / p}\left(U_{+}\right)$and is nondenegerate over $\mathbb{R}$ with respect to the Newton polyhedron $\Gamma_{+}(f)$ (see Definition $10.10(\mathrm{v})$ ).

(i) If the support of $\varphi$ is contained in a sufficiently small neighborhood of the origin, then $\tilde{I}(t ; \varphi)$ admits an asymptotic expansion of the form

$$
\tilde{I}(t ; \varphi) \sim \sum_{\alpha} \sum_{k=1}^{n} C_{\alpha k}(\varphi) t^{\alpha}(\log t)^{k-1} \quad \text { as } t \rightarrow+\infty,
$$

where the progression $\{\alpha\}$ belongs to finitely many arithmetic progressions, which are obtained by using the theory of toric varieties based on the geometry of the Newton polyhedron $\Gamma_{+}\left(F_{f}\right)$, where $F_{f}$ is as in Definition 10.10 (i).

Here the oscillation index of $(f, 1)$ and the multiplicity of its index can be samely defined as in Definition 1.1, which are denoted by $\beta(f, 1), \eta(f, 1)$, respectively.

(ii) $\beta(f, 1) \leq-1 / d(f)$.

(iii) If at least one of the following conditions is satisfied:

(a) $d(f)>1$

(b) $f$ is nonnegative or nonpositive on $U$;

(c) $1 / d(f)$ is not an odd integer and $f_{\tau_{*}}$ does not vanish on $U \cap(\mathbb{R} \backslash\{0\})^{n}$, then $\beta(f, 1)=-1 / d(f)$ and $\eta(f, 1)=m(f)$.

Here the definitions of $d(f), m(f), \tau_{*}, f_{\tau_{*}}$ are given in Definition 10.10 (iv).

Proof. By using the relationships in Section 14.1, Theorems 10.12 and 10.14 easily imply the above theorem.

Remark 14.5. We expect to exchange $\Gamma_{+}\left(F_{f}\right)$ by $\Gamma_{+}(f)$ in Theorem 14.4 (i), but until now we have not yet verified this. (See Remark 10.13.) 


\section{EXAMPLES}

In this section, we give three examples of the phase $f$ and the weight $g$ in the oscillatory integral (1.1), which show the delicate situation around the hypothesis:

(ii-a) $g$ belongs to $\hat{\mathcal{E}}(U)$

in the main theorems in Section 4. It is easy to see that every phase $f$ treated in this section satisfies the conditions (D) and (E) in Section 3. Since the above condition (ii-a) is not needed when $f$ is convenient (see Section 12), we consider only the case when $f$ is not convenient. We always assume that $\varphi$ satisfies the condition $(\mathrm{C})$ in Section 3 .

(I) In the first example, the weight is monomial type as in Section 10. First, this example shows some kind of necessity of the condition (ii-a). Indeed, Example 1 shows the existence of $f, g$ satisfying that $g \notin \hat{\mathcal{E}}(U)$ and $\beta(f, g) \neq-1 / d(f, g)$. On the other hand, in this example, the equation $\beta(f, g)=-1 / d\left(f, x^{p}\right)$ holds even in the case when $g$ is flat in this example. This shows the optimality of the estimate (4.2) in Remark 4.3.

(II) The second example indicates that the hypothesis (ii-a) is too strong. Indeed, Example 2 shows the existence of $f, g$ satisfying that $g \notin \hat{\mathcal{E}}(U)$ but the equalities $\beta(f, g)=-1 / d(f, g)$ and $\eta(f, g)=m(f, g)$ hold. Furthermore, observing this example carefully, it might be expected to weaken the condition (ii-a) by

(ii-a') $g$ belongs to $\hat{\mathcal{E}}\left[\Phi^{-1}\left(\Gamma_{+}(f)\right) \cap \mathbb{R}_{+}^{n}\right](U)$.

(III) Unfortunately, the third example violates this expectation. Indeed, Example 3 shows the existence of $f, g$ satisfying that the condition (ii-a') and $\beta(f, g)=$ $-1 / d(f, g)$ but $\eta(f, g) \neq m(f, g)$.

Remark 15.1. In this remark, we assume that $f, g$ satisfy all the conditions in the assumptions of Theorem 4.4 except the condition (ii).

(1) At present, there is no example of $f, g$ satisfying that the condition (ii-a') holds but the equality $\beta(f, g)=-1 / d(f, g)$ does not hold.

(2) The following modified condition (ii- $\left.\mathrm{a}^{\prime \prime}\right)$ is available for Theorem 4.4,

(ii-a $\left.{ }^{\prime \prime}\right) g$ belongs to $\hat{\mathcal{E}}[Q(f, g)](U)$.

Here $Q(f, g)$ is defined by the convex hull of the set $\Phi^{-1}\left(\Gamma_{+}(f) \backslash \Gamma^{(n-m(f, g)-1)}\right) \cap$ $\mathbb{Z}_{+}^{n}+\mathbb{R}_{+}^{n}$, where $\Gamma^{(k)}$ is the union of the $k$-dimensional faces of $\Gamma_{+}(f)\left(\Gamma^{(-1)}:=\right.$ $\emptyset)$. By the definition, the set $Q(f, g)$ is a polyhedron in $\mathbb{R}_{+}^{n}$ containing $\Gamma_{+}(g)$. Theorem 4.4 can be partially improved by replacing (ii-a) by (ii-a $\left.{ }^{\prime \prime}\right)$. (The proof is omitted.)

15.1. Example 1. Consider the following two-dimensional example with parameters $p, q \in \mathbb{Z}_{+}$and $c \in \mathbb{R}$ :

$$
\begin{aligned}
& f\left(x_{1}, x_{2}\right)=x_{1}^{4}, \\
& g\left(x_{1}, x_{2}\right)=c x_{1}^{2 p} x_{2}^{2 p}+x_{1}^{2 q} x_{2}^{2 q} e^{-1 / x_{2}^{2}}\left(=: g_{1}\left(x_{1}, x_{2}\right)+g_{2}\left(x_{1}, x_{2}\right)\right) .
\end{aligned}
$$

It is easy to see that

- $\Gamma_{+}(f)=\{(4,0)\}+\mathbb{R}_{+}^{2}$.

- If $c \neq 0$, then $\Gamma_{+}(g)=\Gamma_{+}\left(g_{1}\right)=\{(2 p, 2 p)\}+\mathbb{R}_{+}^{2}$.

If $c=0$, then $\Gamma_{+}(g)=\Gamma_{+}\left(g_{2}\right)=\emptyset$ (i.e., $g$ is a nonzero flat function).

- $d(f, g)=\frac{4}{2 p+1}, m(f, g)=1$ if $c \neq 0, d\left(f, x_{1}^{2 q} x_{2}^{2 q}\right)=\frac{4}{2 q+1}$. 
Notice that

$$
\begin{aligned}
g \in \hat{\mathcal{E}}(U) & \Leftrightarrow p \leq q \text { and } c \neq 0 \Leftrightarrow d(f, g)=d\left(f, x_{1}^{2 p} x_{2}^{2 p}\right) \\
& \Leftrightarrow \exists h_{1} \in C^{\infty}(U) \text { with } h_{1}(0) \neq 0 \text { such that } g(x)=x_{1}^{2 p} x_{2}^{2 p} h_{1}(x) ; \\
g \notin \hat{\mathcal{E}}(U) & \Leftrightarrow p>q \text { or } c=0 \\
& \Leftrightarrow \exists h_{2} \in C^{\infty}(U) \text { with } h_{2}(0)=0 \text { such that } g(x)=x_{1}^{2 q} x_{2}^{2 q} h_{2}(x) .
\end{aligned}
$$

We decompose $\tilde{Z}_{ \pm}(s ; \varphi)$ into $\tilde{Z}_{ \pm}^{(1)}(s ; \varphi)+\tilde{Z}_{ \pm}^{(2)}(s ; \varphi)$, where

$$
\tilde{Z}_{ \pm}^{(j)}(s ; \varphi)=\int_{\mathbb{R}_{+}^{2}}(f(x))_{ \pm}^{s} g_{j}(x) \varphi(x) d x, \quad j=1,2 .
$$

Note $\tilde{Z}_{-}^{(j)}(s ; \varphi)=0$ for $j=1,2$. A simple computation gives

$$
\tilde{Z}_{+}^{(1)}(s ; \varphi)=c \int_{0}^{\infty} \int_{0}^{\infty} x_{1}^{4 s+2 p} x_{2}^{2 p} \varphi\left(x_{1}, x_{2}\right) d x_{1} d x_{2} .
$$

Applying Proposition 9.2 we see that the poles of $Z_{+}^{(1)}(s ; \varphi)$ are simple and they are contained in the set $\left\{-\frac{2 p+\nu}{4}: \nu \in \mathbb{N}\right\}$. Similarly, the poles of

$$
\tilde{Z}_{+}^{(2)}(s ; \varphi)=\int_{0}^{\infty} \int_{0}^{\infty} x_{1}^{4 s+2 q} x_{2}^{2 q} e^{-1 / x_{2}^{2}} \varphi\left(x_{1}, x_{2}\right) d x_{1} d x_{2}
$$

are simple and they are contained in the set $\left\{-\frac{2 q+\nu}{4}: \nu \in \mathbb{N}\right\}$. Moreover, applying Proposition 9.3, we see that the coefficient of $\left(s+\frac{2 q+1}{4}\right)^{-1}$ in the Laurent expansion of $\tilde{Z}_{+}^{(2)}(s ; \varphi)$ is

$$
\frac{1}{4} \int_{0}^{\infty} x_{2}^{2 q} e^{-1 / x_{2}^{2}} \varphi\left(0, x_{2}\right) d x_{2}>0 .
$$

From the property of poles of the above functions, we see that the pattern of asymptotic expansion in this case is the same as that in (1.2) even if $g$ is flat. This means that the oscillation index and its multiplicity can be defined in this case.

Needless to say, we can get the same result as in Theorem 4.4 in the case when $g \in \hat{\mathcal{E}}(U)$. Indeed, the inequality $p \leq q$ implies that $\beta(f, g)=-1 / d(f, g)=-\frac{2 p+1}{4}$. Note that if the support of $\varphi$ is sufficiently small, then the value of (15.2) is also very small.

Next, consider when $g \notin \hat{\mathcal{E}}(U)$. When $c \neq 0$, we see that $\beta(f, g)\left(=-\frac{2 q+1}{4}\right)>$ $-1 / d(f, g)\left(=-\frac{2 p+1}{4}\right)$, but $\beta(f, g)=-1 / d\left(f, x_{1}^{2 q} x_{2}^{2 q}\right)\left(=-\frac{2 q+1}{4}\right)$. When $c=0$, in spite of the fact that $g$ is flat, we have $\beta(f, g)=-1 / d\left(f, x_{1}^{2 q} x_{2}^{2 q}\right)\left(=-\frac{2 q+1}{4}\right)$.

As for the multiplicity, it is easy to see that $\eta(f, g)=m(f, g)=1$ when $g$ is nonflat.

15.2. Example 2. Consider the following three-dimensional example with parameters $p, q \in \mathbb{Z}_{+}$:

$$
\begin{aligned}
& f\left(x_{1}, x_{2}, x_{3}\right)=x_{1}^{4}+x_{2}^{4}, \\
& g\left(x_{1}, x_{2}, x_{3}\right)=x_{1}^{2}+x_{1}^{p} x_{2}^{q} e^{-1 / x_{3}^{2}}\left(=: g_{1}\left(x_{1}, x_{2}, x_{3}\right)+g_{2}\left(x_{1}, x_{2}, x_{3}\right)\right) .
\end{aligned}
$$

It is easy to see that

- $\Gamma_{+}(f)=\left\{\alpha \in \mathbb{R}_{+}^{3}: \alpha_{1}+\alpha_{2} \geq 4\right\}$,

- $\Gamma_{+}(g)=\Gamma_{+}\left(g_{1}\right)=\left\{\alpha \in \mathbb{R}_{+}^{3}: \alpha_{1} \geq 2\right\}, \Gamma_{+}\left(g_{2}\right)=\emptyset$,

- $d(f, g)=1, m(f, g)=1$. 
Moreover, we can see that

$$
g \in \hat{\mathcal{E}}(U) \Leftrightarrow p \geq 2, \quad g \notin \hat{\mathcal{E}}(U) \Leftrightarrow p=0,1 .
$$

We decompose $\tilde{Z}_{ \pm}(s ; \varphi)$ into $\tilde{Z}_{ \pm}^{(1)}(s ; \varphi)+\tilde{Z}_{ \pm}^{(2)}(s ; \varphi)$, where

$$
\tilde{Z}_{ \pm}^{(j)}(s ; \varphi)=\int_{\mathbb{R}_{+}^{3}}(f(x))_{ \pm}^{s} g_{j}(x) \varphi(x) d x, \quad j=1,2 .
$$

Note that $\tilde{Z}_{-}^{(j)}(s ; \varphi)=0$ for $j=1,2$ and that

$$
\tilde{Z}_{+}^{(1)}(s ; \varphi)=\int_{\mathbb{R}_{+}^{3}}\left(x_{1}^{4}+x_{2}^{4}\right)^{s} x_{1}^{2} \varphi(x) d x_{1} d x_{2} d x_{3} .
$$

Applying the computation in Sections 10 and 11, the poles of $\tilde{Z}_{+}^{(1)}(s ; \varphi)$ are simple and are contained in the set $\left\{-1-\nu / 4: \nu \in \mathbb{Z}_{+}\right\}$. Moreover, applying Proposition 9.3 , we see that the coefficient of $(s+1)^{-1}$ in the Laurent expansion of $\tilde{Z}_{+}^{(1)}(s ; \varphi)$ is

$$
\frac{1}{4}\left(\int_{0}^{\infty} \frac{y_{1}^{2}}{y_{1}^{4}+1} d y_{1}\right)\left(\int_{0}^{\infty} \varphi\left(0,0, y_{3}\right) d y_{3}\right)
$$

Similar computation implies that the poles of $\tilde{Z}_{+}^{(2)}(s ; \varphi)$ are simple and are contained in the set $\left\{-\frac{p+q+2+\nu}{4}: \nu \in \mathbb{Z}_{+}\right\}$. Moreover, the coefficient of $\left(s+\frac{p+q+2}{4}\right)^{-1}$ in the Laurent expansion of $\tilde{Z}_{+}^{(2)}(s ; \varphi)$ is

$$
\frac{1}{4}\left(\int_{0}^{\infty} \frac{y_{1}^{2}}{\left(y_{1}^{4}+1\right)^{\frac{p+q+2}{4}}} d y_{1}\right)\left(\int_{0}^{\infty} e^{-1 / y_{3}^{2}} \varphi\left(0,0, y_{3}\right) d y_{3}\right) .
$$

Observing (15.3), (15.4), we see that $\beta(f, g)=-1$ holds not only in the case when $p \geq 2(\Leftrightarrow g \in \hat{\mathcal{E}}(U))$ but also in the case when $p+q \geq 2$. Note that if the support of $\varphi$ is sufficiently small, then the value of (15.4) is smaller than that of (15.3). The condition $p+q \geq 2$ is induced from the condition: (ii-a $\left.{ }^{\prime}\right) g$ belongs to $\hat{\mathcal{E}}\left[\Phi^{-1}\left(\Gamma_{+}(f)\right) \cap \mathbb{R}_{+}^{n}\right](U)$. Indeed, $\Phi^{-1}\left(\Gamma_{+}(f)\right) \cap \mathbb{R}_{+}^{n}=\left(\Gamma_{+}(f)-\mathbf{1}\right) \cap \mathbb{R}_{+}^{n}=\{\alpha \in$ $\left.\mathbb{R}_{+}^{2}: \alpha_{1}+\alpha_{2} \geq 2\right\}$.

As for the multiplicity, it is easy to see that $\eta(f, g)=m(f, g)=1$.

15.3. Example 3. Consider the following three-dimensional example:

$$
\begin{aligned}
& f\left(x_{1}, x_{2}, x_{3}\right)=x_{1}^{4} x_{2}^{4} x_{3}^{4}, \\
& g\left(x_{1}, x_{2}, x_{3}\right)=x_{1}^{4} x_{2}^{4} x_{3}^{2}+x_{1}^{2} x_{2}^{2} x_{3}^{4} e^{-1 / x_{3}^{2}}\left(=: g_{1}\left(x_{1}, x_{2}, x_{3}\right)+g_{2}\left(x_{1}, x_{2}, x_{3}\right)\right) .
\end{aligned}
$$

It is easy to see that

- $\Gamma_{+}(f)=\{(4,4,4)\}+\mathbb{R}_{+}^{3}$

- $\Gamma_{+}(g)=\Gamma_{+}\left(g_{1}\right)=\{(4,4,2)\}+\mathbb{R}_{+}^{3}, \Gamma_{+}\left(g_{2}\right)=\emptyset$,

- $d(f, g)=4 / 3, m(f, g)=1$.

Moreover, we can see that

$$
g \notin \hat{\mathcal{E}}(U) \text { but } g \in \hat{\mathcal{E}}\left[\Phi^{-1}\left(\Gamma_{+}(f)\right) \cap \mathbb{R}_{+}^{n}\right](U) .
$$

Note that $\Phi^{-1}\left(\Gamma_{+}(f)\right)=\{(2,2,2)\}+\mathbb{R}_{+}^{3}$.

We decompose $\tilde{Z}_{ \pm}(s ; \varphi)$ into $\tilde{Z}_{ \pm}^{(1)}(s ; \varphi)+\tilde{Z}_{ \pm}^{(2)}(s ; \varphi)$, where

$$
\tilde{Z}_{ \pm}^{(j)}(s ; \varphi)=\int_{\mathbb{R}_{+}^{3}}(f(x))_{ \pm}^{s} g_{j}(x) \varphi(x) d x, \quad j=1,2 .
$$


Note that $\tilde{Z}_{-}^{(j)}(s ; \varphi)=0$ for $j=1,2$ and that

$$
\begin{aligned}
& \tilde{Z}_{+}^{(1)}(s ; \varphi)=\int_{\mathbb{R}_{+}^{3}} x_{1}^{4 s+4} x_{2}^{4 s+4} x_{3}^{4 s+2} \varphi\left(x_{1}, x_{2}, x_{3}\right) d x \\
& \tilde{Z}_{+}^{(2)}(s ; \varphi)=\int_{\mathbb{R}_{+}^{3}} x_{1}^{4 s+2} x_{2}^{4 s+2} x_{3}^{4 s+4} e^{-1 / x_{3}^{2}} \varphi\left(x_{1}, x_{2}, x_{3}\right) d x .
\end{aligned}
$$

Applying Proposition 9.2 we see that the poles of $\tilde{Z}_{+}^{(1)}(s ; \varphi)$ and $\tilde{Z}_{+}^{(2)}(s ; \varphi)$ are contained in the set $\left\{-\frac{3+\nu}{4} ; \nu \in \mathbb{Z}_{+}\right\}$. From the above computation, the order of poles at $s=-3 / 4$ of $\tilde{Z}_{+}^{(1)}(s ; \varphi)$ is not larger than 1 and that of $\tilde{Z}_{+}^{(2)}(s ; \varphi)$ is not larger than 2. Moreover, applying Proposition 9.3, we see that the coefficient of $(s+3 / 4)^{-2}$ in the Laurent expansion of $\tilde{Z}_{+}^{(2)}(s ; \varphi)$ is

$$
\frac{1}{16} \int_{0}^{\infty} x_{3} e^{-1 / x_{3}^{2}} \varphi\left(0,0, x_{3}\right) d x_{3} \neq 0 .
$$

Therefore, we obtain $\beta(f, g)=-1 / d(f, g)=-3 / 4$ and $\eta(f, g)=2>m(f, g)=1$.

\section{ACKNOWLEDGEMENTS}

The authors would like to express their sincere gratitude to Mutsuo Oka and Osamu Saeki for useful discussions about the nondegeneracy condition with respect to the Newton polyhedron and to the referee for a careful reading of the manuscript and for giving the authors many valuable comments. In particular, these comments strongly influenced the contents in Sections 7.1, 10.4, 12.1 and 14.4.

\section{REFERENCES}

[1] V. I. Arnol'd, S. M. Guseřn-Zade, and A. N. Varchenko, Singularities of differentiable maps. Vol. II, Monodromy and asymptotics of integrals, translated from the Russian by Hugh Porteous, translation revised by the authors and James Montaldi, Monographs in Mathematics, vol. 83, Birkhäuser Boston, Inc., Boston, MA, 1988. MR966191 (89g:58024)

[2] M. F. Atiyah, Resolution of singularities and division of distributions, Comm. Pure Appl. Math. 23 (1970), 145-150. MR0256156 (41 \#815)

[3] I. N. Bernštern and S. I. Gel'fand, Meromorphy of the function $P^{\lambda}$ (Russian), Funkcional. Anal. i Priložen. 3 (1969), no. 1, 84-85. MR0247457 (40 \#723)

[4] Edward Bierstone and Pierre D. Milman, Resolution of singularities in Denjoy-Carleman classes, Selecta Math. (N.S.) 10 (2004), no. 1, 1-28, DOI 10.1007/s00029-004-0327-0. MR2061220 (2005c:14074)

[5] Koji Cho, Joe Kamimoto, and Toshihiro Nose, Asymptotic analysis of oscillatory integrals via the Newton polyhedra of the phase and the amplitude, J. Math. Soc. Japan 65 (2013), no. 2, 521-562. MR3055595

[6] Tristan C. Collins, Allan Greenleaf, and Malabika Pramanik, A multi-dimensional resolution of singularities with applications to analysis, Amer. J. Math. 135 (2013), no. 5, 1179-1252, DOI 10.1353/ajm.2013.0042. MR3117305

[7] Jan Denef, Johannes Nicaise, and Patrick Sargos, Oscillating integrals and Newton polyhedra, J. Anal. Math. 95 (2005), 147-172, DOI 10.1007/BF02791501. MR2145563 (2006a:58054)

[8] William Fulton, Introduction to toric varieties, The William H. Roever Lectures in Geometry, Annals of Mathematics Studies, vol. 131, Princeton University Press, Princeton, NJ, 1993. MR.1234037 (94g:14028)

[9] I. M. Gel'fand and G. E. Shilov, Generalized functions. Vol. I: Properties and operations, translated by Eugene Saletan, Academic Press, New York-London, 1964. MR0166596 (29 \#3869) 
[10] Michael Greenblatt, The asymptotic behavior of degenerate oscillatory integrals in two dimensions, J. Funct. Anal. 257 (2009), no. 6, 1759-1798, DOI 10.1016/j.jfa.2009.06.015. MR2540991 (2010j:58060)

[11] Michael Greenblatt, Oscillatory integral decay, sublevel set growth, and the Newton polyhedron, Math. Ann. 346 (2010), no. 4, 857-895, DOI 10.1007/s00208-009-0424-7. MR2587095 (2011f:58043)

[12] Michael Greenblatt, Resolution of singularities, asymptotic expansions of integrals and related phenomena, J. Anal. Math. 111 (2010), 221-245, DOI 10.1007/s11854-010-0016-1. MR2747065(2012d:58041)

[13] Heisuke Hironaka, Resolution of singularities of an algebraic variety over a field of characteristic zero. I, II, Ann. of Math. (2) 79 (1964), 109-203; ibid. (2) 79 (1964), 205-326. MR0199184(33 \#7333)

[14] Isroil A. Ikromov, Michael Kempe, and Detlef Müller, Estimates for maximal functions associated with hypersurfaces in $\mathbb{R}^{3}$ and related problems of harmonic analysis, Acta Math. 204 (2010), no. 2, 151-271, DOI 10.1007/s11511-010-0047-6. MR2653054(2011i:42026)

[15] Isroil A. Ikromov and Detlef Müller, On adapted coordinate systems, Trans. Amer. Math. Soc. 363 (2011), no. 6, 2821-2848, DOI 10.1090/S0002-9947-2011-04951-2. MR2775788 (2012g:58074)

[16] Isroil A. Ikromov and Detlef Müller, Uniform estimates for the Fourier transform of surface carried measures in $\mathbb{R}^{3}$ and an application to Fourier restriction, J. Fourier Anal. Appl. 17 (2011), no. 6, 1292-1332, DOI 10.1007/s00041-011-9191-4. MR2854839 (2012m:42020)

[17] A. Iosevich and E. Sawyer, Maximal averages over surfaces, Adv. Math. 132 (1997), no. 1, 46-119, DOI 10.1006/aima.1997.1678. MR.1488239 (99b:42023)

[18] Jun-ichi Igusa, Forms of higher degree, Tata Institute of Fundamental Research Lectures on Mathematics and Physics, vol. 59, Tata Institute of Fundamental Research, Bombay; by the Narosa Publishing House, New Delhi, 1978. MR.546292 (80m:10020)

[19] Pierre Jeanquartier, Développement asymptotique de la distribution de Dirac attachée à une fonction analytique (French), C. R. Acad. Sci. Paris Sér. A-B 201 (1970), A1159-A1161. MR0420695 (54 \#8707)

[20] Joe Kamimoto and Toshihiro Nose, Toric resolution of singularities in a certain class of $C^{\infty}$ functions and asymptotic analysis of oscillatory integrals, J. Math. Sci. Univ. Tokyo, to appear, arXiv: 1208.3924

[21] Joe Kamimoto and Toshihiro Nose, Asymptotic analysis of weighted oscillatory integrals via Newton polyhedra, Topics in finite or infinite dimensional complex analysis, Tohoku University Press, Sendai, 2013, pp. 3-12. MR3074732

[22] Bernard Malgrange, Intégrales asymptotiques et monodromie (French), Ann. Sci. École Norm. Sup. (4) 7 (1974), 405-430 (1975). MR0372243 (51 \#8459)

[23] Toshihiro Nose, Asymptotic analysis of oscillatory integrals and local zeta functions via Newton polyhedra, Ph.D. Thesis, Kyushu University, 2012.

[24] Toshihisa Okada and Kiyoshi Takeuchi, Meromorphic continuations of local zeta functions and their applications to oscillating integrals, Tohoku Math. J. (2) 65 (2013), no. 2, 159-178, DOI 10.2748/tmj/1372182720. MR.3079283

[25] D. H. Phong and E. M. Stein, The Newton polyhedron and oscillatory integral operators, Acta Math. 179 (1997), no. 1, 105-152, DOI 10.1007/BF02392721. MR1484770 (98j:42009)

[26] Malabika Pramanik and Chan Woo Yang, Decay estimates for weighted oscillatory integrals in $\mathbb{R}^{2}$, Indiana Univ. Math. J. 53 (2004), no. 2, 613-645, DOI 10.1512/iumj.2004.53.2388. MR2060047(2005m:58088)

[27] Helmut Schulz, Convex hypersurfaces of finite type and the asymptotics of their Fourier transforms, Indiana Univ. Math. J. 40 (1991), no. 4, 1267-1275, DOI 10.1512/iumj.1991.40.40056. MR.1142714(93a:42007)

[28] Elias M. Stein, Harmonic analysis: real-variable methods, orthogonality, and oscillatory integrals, with the assistance of Timothy S. Murphy, Monographs in Harmonic Analysis, III, Princeton Mathematical Series, vol. 43, Princeton University Press, Princeton, NJ, 1993. MR.1232192(95c:42002)

[29] Vincent Thilliez, On quasianalytic local rings, Expo. Math. 26 (2008), no. 1, 1-23, DOI 10.1016/j.exmath.2007.04.001. MR2384272(2009m:32007)

[30] A. N. Varchenko, Newton polyhedra and estimation of oscillating integrals, Functional Anal. Appl. 10 (1976), no. 3, 175-196. 
[31] V. A. Vassiliev, Asymptotic behavior of exponential integrals in the complex domain, Functional Anal. Appl. 13 (1979), no. 4, 239-247.

[32] Günter M. Ziegler, Lectures on polytopes, Graduate Texts in Mathematics, vol. 152, SpringerVerlag, New York, 1995. MR,1311028(96a:52011)

Faculty of Mathematics, Kyushu University, Motooka 744, Nishi-Ku, Fukuoka, 8190395, JAPAN

E-mail address: joe@math.kyushu-u.ac.jp

Faculty of Mathematics, Kyushu University, Motooka 744, Nishi-ku, Fukuoka, 8190395, JAPAN

Current address: Faculty of Engineering, Kyushu Sangyo University, Matsukadai 2-3-1, Higashi-ku, Fukuoka, 813-8503, Japan

E-mail address: t-nose@ip.kyusan-u.ac.jp 